\author{
UNIVERSIDADE DE SÃO PAULO \\ INSTITUTO DE FÍSICA
}

\title{
DECAIMENTO DE EXCITAÇÕES QUASE-LIVRES ELEMENTARES: ALARGAMENTO E FLUTUAÇÕES
}

\author{
LÍGIA LIANI BARZ
}

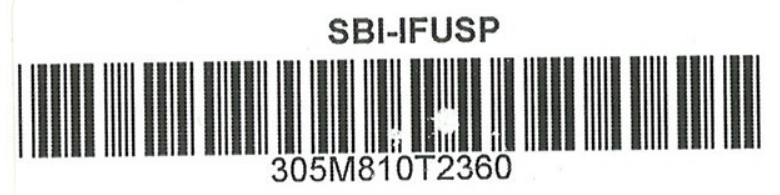

Tese de Doutorado submetida ao Instituto de Física da Universidade de São Paulo

Comissão Examinadora: Prof. Dr. A. F. R. de Toeldo Piza (USP)

Prof. Dr. José Ricardo Marinelli (UFSC)

Prof. Dra. Maria Carolina Nemes (UFMG)

Prof. Dr. Marcos Nogueira Martins (USP)

Prof. Dr. Raphael de Haro Jr. (UFRJ)

ORIENTADOR: Prof. Dr. A. F. R. de TOLEDO PIZA
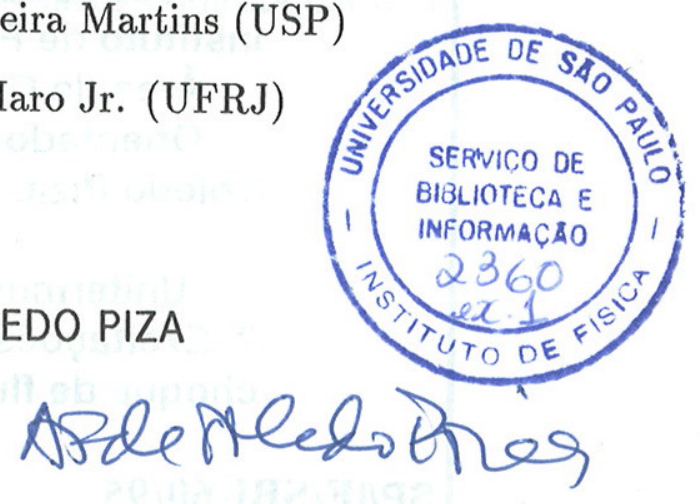

SÃO PAULO

1995

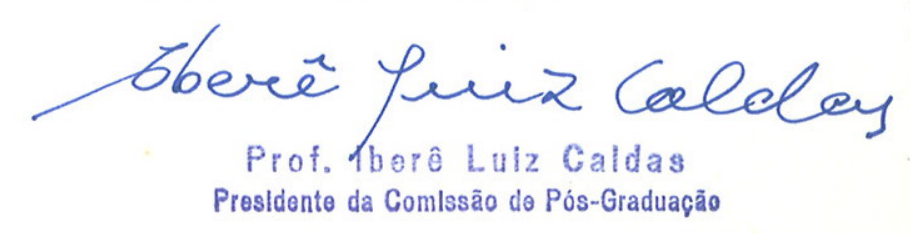




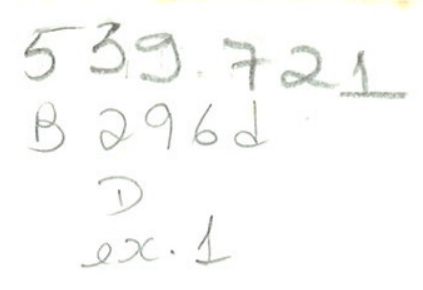

FICHA CATALOGRÁFICA

Preparada pelo Serviço de Biblioteca e Informação do Instituto de Física da Universidade de São Paulo

Barz, Ligia Liani

Decaimento de excitações quase-livres elementares: alargamento e flutuações. IFUSP. São Paulo, 1995.

Tese (Doutoramento) - Universidade de São Paulo. Instituto de Física. Departamento de Física Matemática.

Área de Concentração: Física Nuclear

Orientador: Prof. Dr. Antonio Fernando Ribeiro de Toledo Piza.

Unitermos: 1. Excitações quase-livres elementares; 2. Excitações duas partículas-dois buracos; 3.Seção de choque de flutuação.

SP/IF/SBI-60/95 


\section{Agradecimentos}

Não poderia deixar passar a oportunidade de agradecer algumas pessoas que, ao longo desses anos, nunca deixaram de me dar apoio nos momentos mais críticos.

Quero agradecer especialmente ao meu orientador A. F. R. de Toledo Piza pela sugestão do trabalho, pela dedicação, paciência e pelas explicações valiosas durante esses anos.

Agradeço a minha querida família (pai, mãe, irmãs ...) pelo carinho, compreensão e incentivo.

Agradeço as minhas grandes amigas Hatsumi e Fabiane que sempre encontraram palavras de incentivo nos momentos difíceis.

Agradeço às colegas de sala Adriana, Érica e Karen e ao Luís pela amizade e pela ótima convivência ao longo desses anos.

Agradeço à FAPESP e ao CNPq pelo auxílio financeiro concedido neste período. 


\section{Resumo}

Investigamos os efeitos das excitações duas partículas-dois buracos no espalhamento inelástico de elétrons na região quase-elástica. As funções resposta nuclear longitudinal são obtidas na aproximação do gás de Fermi não ideal. Os aspectos novos do tratamento apresentado, envolvem a escolha de técnicas de discretização que permitem a solução do problema através da inversão sucessiva de matrizes numéricas. Os resultados numéricos são obtidos para o núcleo do ${ }^{56} \mathrm{Fe}$ e a soma coulombiana é calculada.

Em seguida, a seção de choque de espalhamento quase-livre é obtida através da teoria de reações de Feshbach e a representação óptica de fundo. Este tratamento separa a seção de choque en partes lisa e de flutuação. A contribuição de flutuação é expressa em termos de potenciais ópticos usando a teoria de Kerman e McVoy. 


\section{Abstract}

We investigate the effects of two particle-two hole excitations on inelastic electron scattering in the quasi-elastic region. The longitudinal nuclear response functions are obtained in the framework of the Fermi gas model. The new aspects of this treatment, involve the choice of discretization techniques that allow for the solution of the problem through sucessive numerical matrix inversion. Numerical results are obtained for the ${ }^{56} \mathrm{Fe}$ nucleus and the coulomb sum is calculated.

The quasi-free scattering cross section is next obtained using the Feshbach rections theory and the optical-background representation. This treatment separates the cross section into smooth and fluctuating parts. The fluctuation contribution is expressed in terms of optical potentials using the theory of Kerman and McVoy. 


\section{Índice}

1 Introdução 1

2 RPA e Extensões num Esquema Unificado $\quad 7$

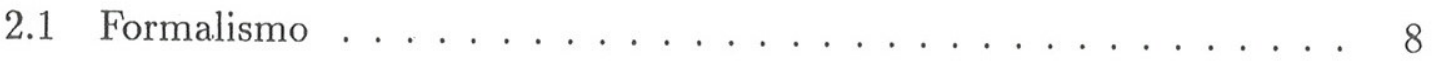

2.1.1 Função resposta baseada na equação de movimento de opera-

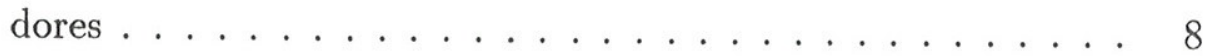

2.1.2 Resposta em termos de operadores de projeção . . . . . . . . 14

2.2 Tratamento numérico . . . . . . . . . . . . . . . 16

2.2.1 RPA partícula-buraco no gás de Fermi . . . . . . . . 16

Interações: . . . . . . . . . . . . . . . . . . . . . . . . 19

I - Interação de Landau-Migdal . . . . . . . . . . . . . . 19

II - Interação Matriz-G . . . . . . . . . . . . . . . 21

Detalhes numéricos e resultados . . . . . . . . . . . . 25

2.2.2 Duas partículas-dois buracos - "SRPA" . . . . . . . . . 30

Detalhes numéricos e resultados . . . . . . . . . . . 35

3 Tratamento das Respostas Eletromagnéticas em Termos de uma Teoria de Reações $\quad 46$

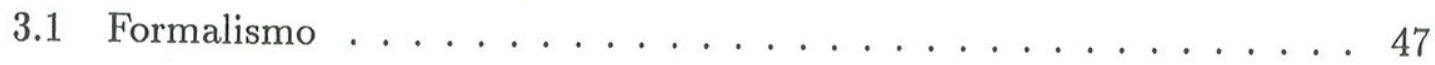

3.1.1 Equações acopladas para $P \Phi_{f}$ e $Q \Phi_{f} \ldots \ldots \ldots$. . . . . . 49 


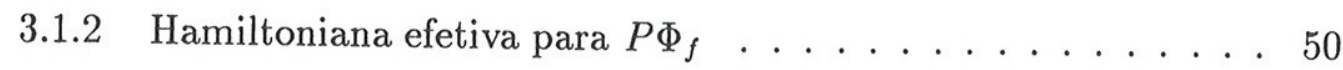

3.1.3 Representação óptica de fundo . . . . . . . . . . . . . 50

3.1.4 Tratamento das flutuações ... . . . . . . . . . . . . 54

4 Conclusões

60

$\begin{array}{ll}\text { A Identidades } & 62\end{array}$

B Matrizes $P H P, P H Q$ e $Q H Q$

C Expressões para determinar o número total de estados $2 \mathrm{p}-2 \mathrm{~h} \quad 68$

D Tratamento de $M_{q R}$ e $g_{f q} \quad 71$

$\begin{array}{ll}\text { Referências } & 74\end{array}$ 


\section{Lista de Figuras}

2.1 Representação diagramática da matriz-G. . . . . . . . . . . . . . . . . 21

2.2 Histograma dos estados de partícula-buraco . . . . . . . . . . . . 26

2.3 Resposta livre como obtida do cálculo da eq. (2.32). . . . . . . . . . 27

2.4 Resposta livre através de cálculo analítico (curva sólida) e através da eq. (2.32) (curva tracejada). . . . . . . . . . . . . . . . 28

2.5 Resposta partícula-buraco com diferentes valores dos parâmetros de Landau-Migdal (RK, RI, LP) e com a interação matriz-G (HM3A). . 29

2.6 Regra de soma coulombiana dividida pelo número atômico, no modelo do Gás de Fermi para a resposta livre, como uma função de $q^{(e)}$ para $k_{F}=1.36 \mathrm{fm}^{-1} \ldots \ldots \ldots \ldots \ldots$

2.7 Representação diagramática das autoenergias. . . . . . . . . . . . 35

2.8 Histograma dos estados duas partículas-dois buracos da autoenergia do buraco no canal $I \equiv\{000\} \ldots \ldots \ldots 6$

2.9 Histograma dos estados duas partículas-dois buracos da autoenergia da partícula no canal $I \equiv\{000\} \ldots \ldots$. . . . . . . . . . 37

2.10 Histograma dos estados duas partículas-dois buracos da autoenergia do buraco no canal $I \equiv\{100\} \ldots \ldots \ldots 8$

2.11 Histograma dos estados duas partículas-dois buracos da autoenergia da partícula no canal $I \equiv\{100\} \ldots \ldots \ldots$. . . . . . . . . 38 
2.12 Histograma da amostra dos estados duas partículas-dois buracos da autoenergia do buraco. . . . . . . . . . . . . . . . . . 39

2.13 Histograma da amostra dos estados duas partículas-dois buracos da autoenergia da partícula. . . . . . . . . . . . . . . . 39

2.14 Resultado do cálculo das equações (2.32) e (2.47) sem a interação p-h (curva sólida) e dados experimentais do ${ }^{56} \mathrm{Fe} . \ldots \ldots$. . . . . 40

2.15 Resposta longitudinal no espaço de $2 \mathrm{p}-2 \mathrm{~h}$ para diferentes atenuação da interação matriz-G. . . . . . . . . . . . . . . . . . . . 40

2.16 Resposta longitudinal $2 \mathrm{p}-2 \mathrm{~h}$ com duas atenuações da interação Matriz-G. . . . . . . . . . . . . . . . . . . . . 41

2.17 Resposta longitudinal $2 \mathrm{p}-2 \mathrm{~h}$ com atenuações da interação matriz-G

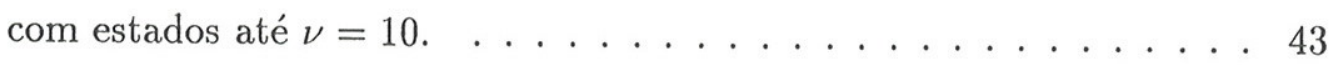

2.18 Resposta longitudinal $2 \mathrm{p}-2 \mathrm{~h}$ com atenuações da interação do tipo Landau-Migdal. . . . . . . . . . . . . . . . . . . . . . 44

3.1 Representação diagramática do processo de espalhamento do elétron. 47 


\section{Lista de Tabelas}

2.1 Parâmetros de Landau-Migdal. . . . . . . . . . . . . . . . 20

2.2 Parametrização da interação matriz-G baseada na versão HM3A. . . . 23

2.3 Resultados da regra de soma coulombiana para o ${ }^{56} \mathrm{Fe} . \ldots \ldots . . .42$ 


\section{Capítulo 1}

\section{Introdução}

Experiências de espalhamento de elétrons sobre o núcleo fornecem uma maneira particularmente direta de estudar vários aspectos da estrutura nuclear e, em particular, de identificar os graus de liberdade mais apropriados na descrição do núcleo. Este papel especial dos elétrons origina-se da constante de acoplamento pequena $(\alpha=1 / 137)$ da interação eletromagnética fundamental. Como resultado é suficiente considerar, numa boa aproximação, apenas o processo de ordem mais baixa (troca de um fóton). Isto porque interações eletromagnéticas que são bem entendidas, permitem uma separação confiável da seção de choque em partes de reação e estrutura nuclear. Além do mais, o elétron interage com o núcleo por meio de um fóton virtual carregando quadri-momento $q_{\mu}=(\imath \omega, \vec{q}), c=1$, que permite variação do momento transferido $\vec{q}$ para uma energia transferida fixa $\omega$ ou vice-versa. Desta maneira pode-se mapear todo o perfil da excitação nuclear. Com fótons reais $\vec{q}$ e $\omega$ são unidos pela condição $q_{\mu} q^{\mu}=0$.

$\mathrm{Na}$ aproximação de troca de um fóton a seção de choque diferencial inclusiva é descrita por

$$
\frac{d^{2} \sigma}{d \Omega d \epsilon}=\sigma_{M}\left[\left(\frac{q_{\mu}^{2}}{|\vec{q}|^{2}}\right)^{2} R_{L}(\vec{q}, \omega)+\left(\frac{1}{2} \frac{q_{\mu}^{2}}{|\vec{q}|^{2}}+\tan ^{2} \frac{\theta}{2}\right) R_{T}(\vec{q}, \omega)\right]
$$


onde $\theta$ denota o ângulo de espalhamento e $\sigma_{M}$ é a seção de choque Mott para o espalhamento por um próton puntual. Os efeitos de estrutura nuclear estão contidos nas funções resposta longitudinal $\left(R_{L}\right)$ e transversa $\left(R_{T}\right)$, relacionadas com as densidades de carga e corrente do núcleo respectivamente.

Muito do que sabemos sobre estrutura nuclear é obtido sondando o núcleo via espalhamento elástico e inelástico de elétrons. Experimentos mais recentes[1]-[6] têm permitido determinar $R_{L}$ e $R_{T}$ separadamente. Estes dados experimentais obtidos do espalhamento inclusivo $\left(e, e^{\prime}\right)$, nos últimos anos, têm atraído a atenção dos físicos nucleares. Há dados disponíveis para vários núcleos: ${ }^{3} \mathrm{He},{ }^{12} \mathrm{C},{ }^{40} \mathrm{Ca},{ }^{48} \mathrm{Ca}$ e ${ }^{56} \mathrm{Fe}$.

O modelo nuclear mais simples possível que pode ser usado para estudar espalhamento inelástico de elétrons é o modelo de gás de Fermi[7]. Neste modelo, as funções de onda nucleares são aproximadas por ondas planas satisfazendo condições de contorno periódicas, normalizadas numa caixa de volume $\mathcal{V}$. Os vários níveis nesta caixa são rotulados por valores específicos do momento linear e cada nível é capaz de conter quatro nucleons (correspondendo às possíveis combinações de spin e isospin) de acordo com o princípio de Pauli. O estado fundamental do núcleo é construído completando estes níveis com os $A$ nucleons do núcleo até um nível representado pelo momento de Fermi nuclear $k_{F}$.

Enquanto a seção de choque parece ser bem descrita pelo modelo do gás de Fermi[8], as correspondentes componentes longitudinal e transversa não são. A resposta longitudinal é muito menor do que os resultados teóricos calculados enquanto a resposta transversa é maior. De todos os modelos propostos para reduzir a intensidade da resposta longitudinal teórica numa maneira desejável, reduz também a resposta transversa numa maneira indesejável. Apesar dos esforços, discrepâncias entre teoria e experimento ainda não foram satisfatoriamente resolvidas.

Para momento transferido $q$ elevado se espera que a soma coulombiana contenha 
a intensidade total da carga do núcleo investigado. Isto realmente é obtido em experimentos com núcleos leves como, por exemplo, o ${ }^{12} C[1]$. Entretanto, com núcleos mais pesados a carga total esperada não foi alcançada. Esta é uma das motivações para extrair a função resposta longitudinal: investigar o "quenching" da regra de soma.

Para isso, recorremos à regra de soma coulombiana (RSC)[9], ou seja, para momento transferido $q$ fixo integramos ao longo da energia $\omega$, a função resposta longitudinal dividida pelo quadrado do fator de forma do nucleon,

$$
R S C=\int \frac{R_{L}(\vec{q}, \omega)}{F_{N}^{2}(q)} d \omega .
$$

A baixas energias, o núcleo atômico pode ser visto como um sistema de nucleons de muitos corpos não relativístico que interage fortemente via troca de mésons virtuais. Apesar de sua complexidade intrínseca, o espectro de excitação mostra muitas características "regulares" que refletem as propriedades médias do núcleo e conduzem ao conceito de campo médio. Tal comportamento regular é sondado no regime da resposta linear onde o núcleo é suavemente perturbado do equilíbrio aplicando um campo externo fraco.

A maior dificuldade na descrição teórica da resposta nuclear está relacionada ao fato que se trata de um sistema de muitos corpos finito com interações de dois corpos complicadas. As soluções analíticas das equações de Schrödinger são conhecidas para alguns casos excepcionais (os sistemas de poucos corpos). É muito improvável que cálculos exatos para núcleos mais pesados seja possível num futuro próximo. Dessa forma, precisamos confiar nas aproximações para reduzir a complexidade das equações de muitos corpos. Tais aproximações, entretanto, devem reter a física essencial e ser consistente com leis de conservação. O campo médio[10] fornece um ponto de partida preciso para as funções de onda de muitos corpos. Baseado na 
noção de campo médio um método que é reconhecido como adequado na descrição da resposta nuclear é a "Randon Phase Approximation" (RPA)[11], que descreve movimento de pequenas oscilações como uma superposição coerente de excitações de uma partícula-um buraco $(1 \mathrm{p}-1 \mathrm{~h})$ construídas a partir do estado fundamental. Este é também, provavelmente, o método mais usado e melhor entendido. A RPA é essencialmente uma teoria de campo médio e é adequada na descrição de energias de excitação médias e probabilidade total de transição. Uma extensão da RPA pela inclusão de excitações $2 \mathrm{p}-2 \mathrm{~h}$ fornece uma teoria mais completa do movimento nuclear de pequenas oscilações. Tal teoria é chamada "Segunda RPA"[12].

Muitas tentativas têm sido feitas para entender as discrepâncias entre teoria e experimento. Elas vão desde tratamentos não relativísticos de muitos corpos convencionais, tais como RPA[13], SRPA[14], ESRPA ("segunda RPA extendida")[15], até teorias relativísticas de campo médio[16]. São também consideradas modificações das propriedades do nucleon[17] no meio nuclear, assim como correntes de troca de mésons[18] (tais como excitações $\Delta$ e produção de píon).

Por outro lado, pode-se obter diretamente expressões para a seção de choque fazendo um tratamento de reações. Neste tratamento a seção de choque de espalhamento inclusiva ou exclusiva pode ser separada numa contribuição "óptica" ou lisa e numa contribuição de flutuação. A contribuição de flutuação não pode ser obtida no tratamento anterior citado (RPA, SRPA, etc). A teoria de reações de Feshbach e a representação óptica de fundo são as técnicas empregadas para investigar o cálculo tradicional[19] das seções de choque de flutuação, que têm sido discutidas nos últimos 30 anos[20]. A representação óptica de fundo é empregada para simplificar a extração de termos de flutuação.

Kawai, Kerman e MacVoy[21] empregaram as técnicas acima em reações do tipo 


$$
A+p \longrightarrow B^{*} \longrightarrow C+c .
$$

Reações deste tipo são ditas responsáveis pela formação do núcleo composto. As seções de choque de flutuação são obtidas diretamente dos elementos da matriz- $S$ óptica.

Contudo, nas reações sequenciais do tipo

$$
\begin{aligned}
A+d \longrightarrow n+B^{*} & \\
& \longrightarrow C+c,
\end{aligned}
$$

as seções de choque de flutuação não podem mais ser obtidas, como na reação anterior, da matriz-S óptica. Apesar disso, Kerman e McVoy[23] mostraram como obter, também nesse caso, a contribuição de flutuação em termos de potenciais ópticos. Estas reações são ditas serem responsáveis pela produção de núcleo composto.

A existência de medidas[22] não inclusivas em reações do tipo $A\left(e, e^{\prime} b\right) X$ mostra a relevância deste tratamento no contexto do espalhamento de elétrons.

$\mathrm{O}$ restante desta tese consiste em três capítulos. No capítulo 2 desenvolvemos o formalismo da função resposta linear que é obtida através da técnica das equações de movimento gerais[12]. Obtemos expressões para as funções resposta longitudinal no espaço de $1 \mathrm{p}-1 \mathrm{~h}$ e $2 \mathrm{p}-2 \mathrm{~h}$ com interações de alcance zero e alcance finito. Em resumo, implementamos o formalismo mencionado anteriormente (RPA e SRPA) no sistema do gás de Fermi e analisamos as contribuições de $1 \mathrm{p}-1 \mathrm{~h}$ e $2 \mathrm{p}-2 \mathrm{~h}$ na resposta nuclear longitudinal. Os cálculos são formados através da escolha de técnicas de discretização e inversão sucessiva de matrizes numéricas. Com a resposta longitudinal no espaço de duas partículas-dois buracos investigamos a regra de soma para o ${ }^{56} \mathrm{Fe}$. 
No capítulo 3 apresentamos o formalismo que permite separar a seção de choque de espalhamento em contribuições lisa e de flutuação. Obtemos uma expressão da seção de choque para as reações do tipo $A\left(e, e^{\prime} b\right) X$, por exemplo,

$$
\begin{aligned}
e+A \longrightarrow e^{\prime}+A^{*} & \\
& \longrightarrow C+p .
\end{aligned}
$$

Finalmente, no capítulo 4 apresentamos as conclusões deste trabalho. 


\section{Capítulo 2}

\section{RPA e Extensões num Esquema Unificado}

Neste capítulo apresentamos uma extensão do método desenvolvido por K. Takayanagi et al.[12] para calcular as funções resposta a partir das equações de movimento de operadores. Incluímos, também, o tratamento acima em termos de operadores de projeção no espaço RPA. Com este formalismo investigamos as funções resposta no espaço de partícula-buraco e duas partícula-dois buracos com interações de alcance zero e alcance finito. 


\subsection{Formalismo}

\subsubsection{Função resposta baseada na equação de movimento de operadores}

A resposta nuclear a uma sonda externa, que é geralmente um operador de um corpo, pode ser investigada na estrutura da teoria RPA. A equação RPA padrão é derivável de muitas maneiras diferentes, e entre elas, o método das equações de movimento é talvez o mais flexível e portanto o mais apropriado para a extensão da teoria RPA. A seguir apresentamos uma particular versão do método das equações de movimento e então desenvolvemos uma forma geral da função resposta baseada no método acima.

Começamos com um conjunto de autoestados $|\nu\rangle$ da hamiltoniana $H$, tal que

$$
H|\nu\rangle=E_{\nu}|\nu\rangle \quad \text { e } \quad H|0\rangle=0
$$

O método RPA (incluindo as chamadas segunda, terceira, extendida, etc) para tratar com a função resposta (veja equação (2.14)) pode ser formulado aproximando as excitações $|\nu\rangle$ como

$$
|\nu\rangle=\Omega_{\nu}^{\dagger}|0\rangle
$$

onde os operadores de excitação $\Omega_{\nu}^{\dagger}$ satisfazem a condição de automodo fraco

$$
\imath \frac{d}{d t}\left\langle 0\left|\left[\Omega_{\mu}(t), \Omega_{\nu}^{\dagger}(0)\right]\right| 0\right\rangle=E_{\nu} \delta_{\mu \nu}\left\langle 0\left|\left[\Omega_{\mu}(t), \Omega_{\nu}^{\dagger}(0)\right]\right| 0\right\rangle, \quad t>0
$$

que leva em conta a exigência da resposta retardada para $\Omega_{\nu}^{\dagger}$ ter uma dependência temporal harmônica ${ }^{1}$. Da equação acima consegue-se, para $t \rightarrow 0^{+}$

\footnotetext{
${ }^{1}$ Automodos fortes: a dependência do operador de Heisenberg $\Omega_{\nu}^{\dagger}(t)$ é harmônica
} 


$$
\left\langle 0\left|\left[\left[\Omega_{\mu}, H\right], \Omega_{\nu}^{\dagger}\right]\right| 0\right\rangle=E_{\nu} \delta_{\mu \nu}\left\langle 0\left|\left[\Omega_{\mu}, \Omega_{\nu}^{\dagger}\right]\right| 0\right\rangle
$$

ou, usando a identidade de Jacobi no lado esquerdo e $H|0\rangle=0$,

$$
\left\langle 0\left|\left[\Omega_{\mu},\left[H, \Omega_{\nu}^{\dagger}\right]\right]\right| 0\right\rangle=E_{\nu} \delta_{\mu \nu}\left\langle 0\left|\left[\Omega_{\mu}, \Omega_{\nu}^{\dagger}\right]\right| 0\right\rangle
$$

Diferentes aproximações de tal família são agora geradas expandindo os $\Omega_{\nu}^{\dagger}$ nas bases de operador truncado $\left\{A_{i}^{\dagger}, A_{i}\right\}$ :

$$
\Omega_{\nu}^{\dagger}=\sum_{i}\left(X_{i}^{\nu} A_{i}^{\dagger}-Y_{i}^{\nu} A_{i}\right) .
$$

O sinal menos é colocado por convenção na teoria RPA. Usando (2.5) em (2.4) obtemos a seguinte equação matricial

$$
\left(\begin{array}{ll}
X^{\dagger} & Y^{\dagger}
\end{array}\right)\left(\begin{array}{cc}
A & -B \\
-B^{*} & A^{*}
\end{array}\right)\left(\begin{array}{c}
X \\
Y
\end{array}\right)=\left(\begin{array}{ll}
X^{\dagger} & Y^{\dagger}
\end{array}\right)\left(\begin{array}{cc}
S & -T \\
T^{*} & -S^{*}
\end{array}\right)\left(\begin{array}{c}
X \\
Y
\end{array}\right) \varepsilon
$$

onde $\varepsilon$ é a matriz de "autovalor" diagonal e

$$
\begin{aligned}
(A)_{i j} & \equiv\left\langle 0\left|\left[A_{i},\left[H, A_{j}^{\dagger}\right]\right]\right| 0\right\rangle ; & & A=A^{\dagger} \\
(B)_{i j} & \equiv\left\langle 0\left|\left[A_{i},\left[H, A_{j}\right]\right]\right| 0\right\rangle ; & B & =B^{T} \\
(S)_{i j} & \equiv\left\langle 0\left|\left[A_{i}, A_{j}^{\dagger}\right]\right| 0\right\rangle ; & & S=-S^{\dagger} \\
(T)_{i j} & \equiv\left\langle 0\left|\left[A_{i}, A_{j}\right]\right| 0\right\rangle ; & & T=-T^{T} .
\end{aligned}
$$

É importante observar que $A_{i j}=A_{j i}^{*}$ se $H|0\rangle=0$ (equação (2.1)). Esta última relação implica $\left\langle 0\left|a_{h_{i}}^{\dagger} a_{p_{i}} a_{p_{j}}^{\dagger} a_{h_{j}} H\right| 0\right\rangle=0$, mas isto não se mantém exatamente se $|0\rangle$ é um estado fundamental aproximado. Neste caso, há duas maneiras de forçar a hermiticidade de $A_{i j}$ : 
1. Ignorar os elementos de matriz nos quais $H$ age diretamente sobre $|0\rangle$,

2. Trocar os comutadores duplos por comutadores duplos simetrizados de Rowe, ou seja,

$$
\left[A_{i},\left[H, A_{j}^{\dagger}\right]\right] \longrightarrow\left[A_{i}, H, A_{j}^{\dagger}\right] \equiv \frac{1}{2}\left[A_{i},\left[H, A_{j}^{\dagger}\right]\right]+\frac{1}{2}\left[\left[A_{i}, H\right], A_{j}^{\dagger}\right] .
$$

É conveniente escrever a equação (2.6) numa forma mais curta. Assim, introduzindo uma matriz $\mathcal{G}=\left(\begin{array}{cc}1 & 0 \\ 0 & -1\end{array}\right)$ e as notações

$$
\begin{gathered}
\mathcal{U}=\mathcal{G}\left(\begin{array}{cc}
A & -B \\
-B^{*} & A^{*}
\end{array}\right) \mathcal{G}=\left(\begin{array}{cc}
A & B \\
B^{*} & A^{*}
\end{array}\right) \\
\mathcal{N}=\mathcal{G}\left(\begin{array}{cc}
S & -T \\
T^{*} & -S^{*}
\end{array}\right) \mathcal{G}=\left(\begin{array}{cc}
S & T \\
-T^{*} & -S^{*}
\end{array}\right) \\
\mathcal{Z} \equiv \mathcal{G}\left(\begin{array}{c}
X \\
Y
\end{array}\right)=\left(\begin{array}{c}
X \\
-Y
\end{array}\right),
\end{gathered}
$$

e cancelando o fator mais à esquerda de cada lado da equação de autovalor (2.6), obtemos

$$
\mathcal{U Z}=\mathcal{N} \mathcal{Z} \mathcal{E} .
$$

Até agora a suposição feita no modelo sobre o estado fundamental é que $H|0\rangle=0$. Os esquemas RPA "elevados" (segunda, terceira, etc) geralmente adotam um modelo de Hartree-Fock para $|0\rangle$, enquanto esquemas RPA "extendidos, elevados" acrescentam correlações de estado fundamental perturbativos, embora nenhum dos dois satisfaça a condição $H|0\rangle=0$. Um ponto que deve ser enfatizado é que os esquemas "extendidos" mudam a estrutura do estado fundamental $|0\rangle$. Por exemplo, pode-se propor um estado fundamental perturbativamente correlacionado com componentes duas partículas-dois buracos: $|0\rangle=|H F\rangle-\sum_{2_{0}} \frac{\left\langle p_{1} p_{2}|V| h_{1} h_{2}\right\rangle}{\epsilon_{p_{1}}+\epsilon_{p_{2}}-\epsilon_{h_{1}}-\epsilon_{h_{2}}} a_{p_{1}}^{\dagger} a_{p_{2}}^{\dagger} a_{h_{1}} a_{h_{2}}|H F\rangle$, 
onde $2_{0} \equiv p_{1} p_{2} h_{1} h_{2}$. Os esquemas "elevados", por sua vez, aumentam a base de operadores $\left\{A_{i}\right\}$. Por exemplo, de uma base de partícula-buraco, $a_{h_{i}}^{\dagger} a_{p_{i}}$, para uma de duas partículas-dois buracos, $a_{h_{i 1}}^{\dagger} a_{h_{i 2}}^{\dagger} a_{p_{i 1}} a_{p_{i 2}}$.

Podemos agora discutir algumas propriedades formais da equação (2.11). As soluções da equação acima emergem em pares opostos, isto é, se $\left(X_{i}^{\nu}, Y_{i}^{\nu}\right)$ constitui um autovetor com autovalor $E_{\nu}$, então $\left(Y_{i}^{\nu \dagger}, X_{i}^{\nu \dagger}\right)$ constitui um autovetor com autovalor $-E_{\nu}$.

A normalização do vetor $\mathcal{Z}$ é dada por

$$
\mathcal{Z}^{\dagger} \mathcal{N} \mathcal{Z}=\mathcal{G} \equiv\left(\begin{array}{cc}
1 & 0 \\
0 & -1
\end{array}\right)
$$

Além disso, os autovetores satisfazem a relação de completeza

$$
\mathcal{Z} \mathcal{Z}^{\dagger} \mathcal{N}=\mathcal{N} \mathcal{Z} \mathcal{Z}^{\dagger}=1
$$

Agora estamos prontos para discutir a função resposta de um sistema que é governado pela equação (2.1). A função resposta correspondente ao operador externo $\hat{\mathcal{O}}$ é definida como[24]

$$
\begin{aligned}
R(\omega) & =-\imath \int_{-\infty}^{\infty} d t e^{\imath \omega t}\left\langle 0\left|\left[\hat{\mathcal{O}}_{H}^{\dagger}(t), \hat{\mathcal{O}}_{H}(0)\right]\right| 0\right\rangle \theta(t) \\
& =\sum_{\nu}\left[\frac{\left\langle 0\left|\mathcal{O}^{\dagger}\right| \nu\right\rangle\langle\nu|\mathcal{O}| 0\rangle}{\omega-E_{\nu}+\imath \eta}-\frac{\langle 0|\mathcal{O}| \nu\rangle\left\langle\nu\left|\mathcal{O}^{\dagger}\right| 0\right\rangle}{\omega+E_{\nu}+\imath \eta}\right]
\end{aligned}
$$

numa representação de Lehman (representação espectral) e $\eta$ é um número infinitesimal positivo. $\mathrm{O}$ operador de Heisenberg $\hat{\mathcal{O}}$ acima é definido como $\hat{\mathcal{O}}_{H}=e^{\imath H t} \mathcal{O}^{-\imath H t}$.

Assumimos que o operador $\mathcal{O}$ pode também ser expandido no conjunto $\left\{A_{i}^{\dagger}, A_{i}\right\}$, exatamente como $\Omega_{\nu}^{\dagger}$ (veja equação $\left.(2.5)\right)$ : 


$$
\mathcal{O}=\sum_{i}\left(C_{i} A_{i}^{\dagger}-D_{i} A_{i}\right)
$$

Os operadores $\Omega_{\nu}^{\dagger}$, por outro lado, podem ser obtidos de $\Lambda=\mathcal{Z}^{\dagger} \mathcal{A}$, onde $\mathcal{A}$ é o vetor coluna contendo os $\left(A_{i}^{\dagger}, A_{i}\right)$ e $\Lambda$ é uma coluna contendo os $\Omega_{\nu}^{\dagger}$. Usando a relação de completeza, $(2.13)$, obtemos

$$
\mathcal{A}=\mathcal{N} \mathcal{Z} \mathcal{G} \mathcal{Z}^{\dagger} \mathcal{A}=\mathcal{N} \mathcal{Z} \mathcal{G} \Lambda
$$

o que nos dá os $\left(A_{i}^{\dagger}, A_{i}\right)$ em termos dos automodos fracos $\Omega_{\nu}^{\dagger}$. Reescrevendo a equação (2.15) como $\mathcal{O}=\mathrm{O}^{\dagger} \mathcal{A}$ conseguimos finalmente

$$
\mathcal{O}=\mathrm{O}^{\dagger} \mathcal{N} \mathcal{Z} \mathcal{G} \Lambda \equiv \sum_{\nu} o_{\nu} \Omega_{\nu}^{\dagger} \equiv \mathbf{o}^{\dagger} \Lambda
$$

o que identifica $\mathrm{O}^{\dagger} \mathcal{N} \mathcal{Z} \mathcal{G}$ como os coeficientes de expansão de $\mathcal{O}$ em termos dos automodos fracos.

A equação (2.17) pode também ser escrita como

$$
\mathcal{O}=\sum_{E_{\nu}>0}\left(o_{\nu} \Omega_{\nu}^{\dagger}-o_{-\nu} \Omega_{\nu}\right)
$$

onde $o_{-\nu}$ corresponde a energias negativas no somatório da equação (2.17).

Com a ajuda desta expansão podemos reescrever o termo que aparece no integrando de $R(\omega)$, equação (2.14), como:

$$
\begin{aligned}
& \left\langle 0\left|\left[\hat{\mathcal{O}}_{H}^{\dagger}\left(0^{+}\right), \hat{\mathcal{O}}_{H}(0)\right]\right| 0\right\rangle= \\
& =\sum_{\substack{E_{\nu}>0 \\
E_{\mu}>0}}\left\{-o_{\nu}^{*} o_{-\mu}\left\langle 0\left|\left[\Omega_{\nu}\left(0^{+}\right), \Omega_{\mu}(0)\right]\right| 0\right\rangle-o_{-\nu}^{*} o_{\mu}\left\langle 0\left|\left[\Omega_{\nu}^{\dagger}\left(0^{+}\right), \Omega_{\mu}^{\dagger}(0)\right]\right| 0\right\rangle\right. \\
& \left.\quad+o_{\nu}^{*} o_{\mu}\left\langle 0\left|\left[\Omega_{\nu}\left(0^{+}\right), \Omega_{\mu}^{\dagger}(0)\right]\right| 0\right\rangle+o_{-\nu}^{*} o_{-\mu}\left\langle 0\left|\left[\Omega_{\nu}^{\dagger}\left(0^{+}\right), \Omega_{\mu}(0)\right]\right| 0\right\rangle\right\}
\end{aligned}
$$


A partir da equação acima, obtemos

$$
R(\omega)=\sum_{E_{\nu}>0}\left[\frac{\left|o_{\nu}\right|^{2}}{\omega-E_{\nu}+\imath \eta}-\frac{\left|\tilde{o}_{\nu}\right|^{2}}{\omega+E_{\nu}+\imath \eta}\right]=\mathbf{o}^{\dagger} \frac{\mathcal{G}}{(\omega+\imath \eta) \mathbf{1}-\mathcal{E}} \mathbf{o} .
$$

Isto mostra como a resposta retardada aparece no método RPA (generalizado, extendido) em termos dos correspondentes modos fracos e suas respectivas frequências. O cálculo de (2.20) involve a solução geral do problema de diagonalização (2.11), que pode ser uma tarefa impraticável. Entretanto se $\mathcal{O}(2.15)$ contém apenas um pequeno subconjunto dos $\left(A_{i}^{\dagger}, A_{i}\right)$ então pode-se recorrer às técnicas de projeção para reduzir as expressões analíticas antes do cálculo numérico.

Voltando à equação (2.11), que é a equação dinâmica básica da aproximação RPA, segue que

$$
[(\omega+\imath \eta) \mathcal{N}-\mathcal{U}] \mathcal{Z}=\mathcal{N} \mathcal{Z}[(\omega+\imath \eta) \mathbf{1}-\mathcal{E}]
$$

de modo que

$$
\mathcal{Z}[(\omega+\imath \eta) \mathbf{1}-\mathcal{E}]^{-1}=[(\omega+\imath \eta) \mathcal{N}-\mathcal{U}]^{-1} \mathcal{N} \mathcal{Z}
$$

Multiplicando $\mathcal{Z}^{\dagger} \mathcal{N}$ pela esquerda em ambos os lados e usando a condição de normalização, equação (2.12), conseguimos finalmente

$$
\frac{\mathcal{G}}{(\omega+\imath \eta) \mathbb{1}-\mathcal{E}}=\mathcal{Z}^{\dagger} \mathcal{N} \frac{1}{(\omega+\imath \eta) \mathcal{N}-\mathcal{U}} \mathcal{N} \mathcal{Z}
$$

Com isso podemos reescrever a resposta $R(\omega)$ (equação (2.20)) como,

$$
R(\omega)=\mathbf{o}^{\dagger} \mathcal{Z}^{\dagger} \mathcal{N} \frac{1}{(\omega+\imath \eta) \mathcal{N}-\mathcal{U}} \mathcal{N} \mathcal{Z} \mathbf{o}
$$

Usando a definição (2.17) de o $^{\dagger}$ e a relação de completeza, obtemos 


$$
\mathbf{o}^{\dagger} \mathcal{Z}^{\dagger} \mathcal{N}=\mathrm{O}^{\dagger} \mathcal{N} \mathcal{Z} \mathcal{G} \mathcal{Z}^{\dagger} \mathcal{N}=\mathrm{O}^{\dagger} \mathcal{N}
$$

Dessa forma,

$$
R(\omega)=\mathrm{O}^{\dagger} \mathcal{N} \frac{1}{(\omega+\imath \eta) \mathcal{N}-\mathcal{U}} \mathcal{N O}
$$

lembrando que $\mathcal{O}=O^{\dagger} \mathcal{A}$.

A expressão final acima é a forma geral da função resposta derivada pela equação de movimento de operadores. Observe que modificamos o problema de autovalor em um problema de inversão de matriz, $[(\omega+\imath \eta) \mathcal{N}-\mathcal{U}]^{-1}$.

\subsubsection{Resposta em termos de operadores de projeção}

A inversão do operador envolvida nesta forma de $R(\omega)$, equação (2.26), pode ser agora tratada com as técnicas de projeção padrão desenvolvidas por Feshbach em conexão com a teoria de reações nucleares.

Para ilustrar isso, vamos assumir que o espaço de trabalho inteiro é dividido em dois subespaços mutuamente ortogonais associados com os projetores $P$ e $Q$ (veja seção 3.1 .1 ), isto é,

$$
\begin{gathered}
1=P+Q, \\
P=P^{\dagger}=P^{2}, \quad Q=Q^{\dagger}=Q^{2}, P Q=Q P=0 .
\end{gathered}
$$

A técnica de operadores de projeção tem provado ser bastante geral e útil. 0 conceito básico do formalismo é a divisão do espaço de Hilbert em subespaços cada um com suas características. Permite-se, então, a interação entre os subespaços para gerar as características da Hamiltoniana inteira. A vantagem do método é 
imediata: podemos, por exemplo, escolher somente os subespaços que são diretamente importantes num problema específico para tratamento explícito e tratar fenomenologicamente os acoplamentos aos subespaços relevantes. As interações de acoplamento agem algumas vezes de forma que o seu efeito médio pode ser descrito de forma simples (confira capítulo 3). Naturalmente qualquer estado físico contém uma combinação linear dos estados de todos os subespaços, que estão dinamicamente acoplados.

Com a ajuda das identidades de operador padrão[25] (veja apêndice A) pode-se, neste caso, reescrever a equação (2.26) como:

$$
\begin{aligned}
R(\omega) & =\mathrm{O}^{\dagger} \mathcal{N} Q \frac{1}{W_{Q Q}} Q \mathcal{N O} \\
& +\mathrm{O}^{\dagger} \mathcal{N}\left(P+Q \frac{1}{W_{Q Q}} W_{Q P}\right) \frac{1}{W_{P P}-W_{P Q} \frac{1}{W_{Q Q}} W_{Q P}}\left(P+W_{P Q} \frac{1}{W_{Q Q}} Q\right) \mathcal{N O}
\end{aligned}
$$

onde $W=(\omega+\imath \eta) \mathcal{N}-\mathcal{U}, W_{P P}=P W P, W_{P Q}=P W Q, W_{Q Q}=Q W Q$ e $W_{Q P}=$ $Q W P$.

Note que as projeções realizadas acima correspondem a uma decomposição do operador unidade como implementada na relação de completeza: $1=\mathcal{Z} \mathcal{G Z}^{\dagger} \mathcal{N}$. Fisicamente isto corresponde a uma decomposição do subespaço do espaço linear dos modos de excitação permitidos pela equação (2.5). Visto que o esquema tipo-RPA envolve uma métrica que, por sua vez, envolve explicitamente um estado (fundamental) $|0\rangle$ de referência, e visto que isto não precisa ser um estado Hartree-Fock (ele poderia incluir correlações de estado fundamental de alguma espécie), esta decomposição não corresponde em geral a uma decomposição similar em termos das configurações de muitas partículas envolvidas nas excitações. Por exemplo, correlações duas partículas-dois buracos em $|0\rangle$, sob a ação de um operador de um corpo, darão configurações partícula-buraco, duas partículas-dois buracos e três partículas-três 
buracos. Devido a esta circunstância, a correspondência entre os processos incluídos num esquema RPA extendido e uma análise diagramática da teoria de perturbação de muitos corpos não é trivial e crucialmente dependente da extensão particular sobre a qual se baseia o esquema RPA.

\subsection{Tratamento numérico}

O trabalho que desenvolvemos envolve um tratamento numérico diferente do usual para o estudo da resposta nuclear a projéteis eletromagnéticos, especialmente na região do pico quase-elástico, que permite em particular a inclusão completa de efeitos da interação residual no espaço de duas partículas-dois buracos (2p-2h). Os aspectos especificamente novos desse tratamento, envolvem a escolha de técnicas de discretização que permitem a solução do problema através da inversão sucessiva de matrizes numéricas.

Estamos interessados em investigar a função resposta longitudinal partículaburaco (p-h) quando forças de alcance zero e alcance finito são adotadas como as interações residuais. Um aperfeiçoamento disto é feito quando introduzimos os graus de liberdade duas partículas-dois buracos. As expressões da função resposta, tanto partícula-buraco quanto duas partículas-dois buracos, são obtidas diretamente da forma geral (equação (2.26)) encontrada na seção (2.1.1).

\subsubsection{RPA partícula-buraco no gás de Fermi}

Vamos obter expressões para a resposta longitudinal partícula-buraco no modelo do gás de Fermi. Como mencionado no capítulo 1, no modelo do gás de Fermi as funções de onda são simplesmente ondas planas que satisfazem condições de contorno periódicas, normalizadas numa caixa de volume $\mathcal{V}$. Os vários níveis nesta caixa são 
rotulados por valores específicos do momento linear e, por causa do princípio de Pauli, cada estado de momento (nível) pode ser ocupado por no máximo quatro nucleons (correspondendo aos spin e isospin "up" e "down"). O estado fundamental do núcleo é construído completando estes níveis com os $A$ nucleons do núcleo até o nível de Fermi.

A RPA é considerada a teoria apropriada para investigar interações entre partículas e buracos. Entretanto, para simplificar o tratamento numérico explícito descrito a seguir, nós nos restringimos à aproximação Tamm-Dancoff (TDA). Neste método considera-se $B=0$ na equação $(2.7 \mathrm{~b})$. Vamos assumir uma base de operador de criação e destruição de partícula-buraco $\left\{A_{i}^{\dagger}, A_{i}\right\}$ sendo $A_{i}=a_{h_{i}}^{\dagger} a_{p_{i}}$ e $A_{i}^{\dagger}=a_{p_{i}}^{\dagger} a_{h_{i}}$, e aproximar o estado fundamental como sendo o de Hartree-Fock $(|0\rangle=|H F\rangle)$. Assim, obtemos

$$
\begin{aligned}
& (A)_{i j}=\left\langle H F\left|A_{j} H A_{i}^{\dagger}\right| H F\right\rangle=\left\langle p_{j} h_{j}|H| p_{i} h_{i}\right\rangle \equiv P H P \\
& (T)_{i j}=0, \quad(S)_{i j}=1 .
\end{aligned}
$$

Usando isto e as técnicas de projeção de Feshbach da seção (2.1.2), levando em conta que $\mathrm{O}$ é um operador de um corpo, conseguimos uma expressão para a função resposta, que corresponde ao termo $\mathrm{O}^{\dagger} \mathcal{N} P \frac{1}{W_{P P}} P \mathcal{N} O$ na equação $(2.28)$, dado por $^{2}$

$$
R(\omega)=\sum_{\substack{p_{i}, h_{i} \\ p_{j}, h_{j}}}\left\langle H F\left|\mathrm{O}^{*}\right| p_{j} h_{j}\right\rangle\left\langle p_{j} h_{j}\left|\frac{1}{\omega+\imath \eta-P H P}\right| p_{i} h_{i}\right\rangle\left\langle p_{i} h_{i}|\mathrm{O}| H F\right\rangle
$$

sendo $\mathrm{O}=\sum_{\alpha \beta}\langle\alpha|o| \beta\rangle a_{\alpha}^{\dagger} a_{\beta}$ o operador de um corpo e $h \equiv\left(\vec{k}_{h}, m_{s_{h}}, m_{t_{h}}\right)$ significa momento e projeções de spin e isospin do buraco $(h \rightarrow p$, da partícula). Introduzindo

\footnotetext{
${ }^{2}$ Obtemos $P H P$ e não $P W P$ na equação (2.30) porque consideramos apenas o primeiro elemento das matrizes $\mathcal{U}$ e $\mathcal{N}$. É redundante considerar $A$ e $A^{*}$ em $\mathcal{U}$ (veja equação (2.8)), assim como $S$ e $-S^{*}$ em $\mathcal{N}$.
} 
a notação de spin e isospin completa, escrevemos PHP (apêndice B) como

$$
P H P \equiv\left\langle\left[p_{j} h_{j}\right] S M_{S} T M_{T}|H|\left[p_{i} h_{i}\right] S M_{S} T M_{T}\right\rangle
$$

O que nos interessa é a parte imaginária da função resposta que está relacionada com a probabilidade total de transição de um operador de um corpo do estado fundamental para um estado excitado $\langle 0|\mathcal{O}| \nu\rangle$ (equação (2.14)):

$$
R^{I}(\nu)=-\frac{1}{\pi} \frac{m}{\hbar^{2} k_{F}^{2}} \Im m\left[\sum_{i j} M_{i j}^{I}(\nu+\imath \eta)\right]
$$

onde

$$
\begin{aligned}
M_{i j}^{I}(\nu+\imath \eta)= & \frac{8 k_{F}^{3}}{15 \pi^{2} N} \sum_{\substack{k_{p}^{\prime}, k_{h}^{\prime} \\
k_{p}, k_{h}}} \delta_{\vec{k}_{p}^{\prime}, \vec{k}_{h}^{\prime}+\vec{q}^{(e)}} \theta\left(k_{p}^{\prime}-k_{F}\right) \theta\left(k_{F}-k_{h}^{\prime}\right) \\
& \times\left[\frac{1}{\nu+\imath \eta-P \mathcal{H} P}\right]_{\substack{k_{p}^{\prime} k_{h}^{\prime} \\
k_{p} k_{h}}}^{I} \delta_{\vec{k}_{p}, \vec{k}_{h}+\vec{q}^{\prime}(e)} \theta\left(k_{p}-k_{F}\right) \theta\left(k_{F}-k_{h}\right) .
\end{aligned}
$$

As funções $\theta$ asseguram que o buraco está dentro do mar de Fermi $\left(k_{h}<k_{F}\right)$ e a partícula fora $\left(k_{p}>k_{F}\right)$. Na equação acima $I \equiv\left\{T S M_{S}\right\}$ significa, respectivamente, isospin, spin e projeção de spin. O cálculo da resposta nos canais $I \equiv\{000\}$ e $I \equiv\{100\}$ dá a resposta longitudinal $\left(R_{L}=R^{000}+R^{100}\right)$. O fator constante na frente da equação foi colocado para que o resultado da resposta livre, neste cálculo, coincida com o resultado obtido através de um cálculo analítico (neste caso, com o do Fetter-Walecka[24]). O parâmetro $\nu=\omega \frac{m}{\hbar^{2} k_{F}^{2}}$ é adimensional e $\mathcal{H}=H / 2 \epsilon_{F}$, sendo $\epsilon_{F}$ a energia de Fermi. O número de estados de partícula-buraco é dado por $N$. Usamos ainda um operador o de ondas planas, com momento transferido $\vec{q}^{(e)}$,

$$
o=e^{2 \vec{q}^{(e)} \cdot \vec{r}} .
$$


Os deltas de Kroenecker na equação (2.33) vêm do cálculo dos elementos de matriz na base de ondas planas.

A hamiltoniana microscópica $H$ consiste de um termo de energia cinética e interação de dois corpos. Por conveniência vamos escrevê-la em segunda quantização

$$
H=\sum_{\alpha} \epsilon_{\alpha} a_{\alpha}^{\dagger} a_{\alpha}+\frac{1}{2} \sum_{\alpha \beta \gamma \delta}\langle\alpha \beta|V| \gamma \delta\rangle a_{\alpha}^{\dagger} a_{\beta}^{\dagger} a_{\delta} a_{\gamma}
$$

\section{Interações:}

Existe um número grande de diferentes interações fenomenológicas que são aplicadas em problemas de Física Nuclear. Cada uma delas é usada num problema específico e seus alcances de validade e sucesso variam muito.

O cálculo mais simples que podemos fazer da resposta, consiste em usar uma força de alcance zero. De fato, tais forças tornam-se úteis porque elas são muito simples de se tratar e descrevem propriedades nucleares muito bem. Forças mais realísticas, entretanto, precisam ter um alcance finito.

Nós nos restringimos, neste trabalho, às interações de Landau-Migdal de alcance zero e matriz-G de alcance finito.

I - Interação de Landau-Migdal - O nosso modelo nuclear nos permite usar uma interação residual de alcance zero dada por,

$$
V=C_{o}\left(F_{o}+F_{o}^{\prime} \vec{\tau}_{1} \cdot \vec{\tau}_{2}+G_{o} \vec{\sigma}_{1} \cdot \vec{\sigma}_{2}+G_{o}^{\prime} \vec{\sigma}_{1} \cdot \vec{\sigma}_{2} \vec{\tau}_{1} \cdot \vec{\tau}_{2}\right) \delta\left(\vec{r}_{1}-\vec{r}_{2}\right) .
$$

Aqui $C_{o}$ é um parâmetro de intensidade e $F_{o}$, etc são os parâmetros de LandauMigdal que são geralmente ajustados para alguns estados excitados conhecidos (tabela (2.1)). É importante notar que esta é uma força p-h efetiva e que não 
tem de ser antissimetrizada[26]. Usando isto obtemos uma expressão para $P \mathcal{H} P$ que substituída nas equações (2.33) e (2.32) dá a resposta longitudinal p-h,

Tabela 2.1: Parâmetros de Landau-Migdal.

\begin{tabular}{||c|c|c|c|c|c||}
\hline \hline & $C_{o}$ & $F_{o}$ & $F_{o}^{\prime}$ & $G_{o}$ & $G_{o}^{\prime}$ \\
\hline \hline RK & 253.531 & 0.2 & 1.5 & 0.55 & 0.7 \\
\hline RI & 380.0 & 0.0685 & 0.3315 & 0.575 & 0.725 \\
\hline \hline LP & 65.0 & 0.2 & 1.5 & 0.55 & 0.7 \\
\hline \hline
\end{tabular}

$C_{o}$ é dado em $\mathrm{MeV} \mathrm{fm}^{3}$ e $F_{o}$, etc são adimensionais. RK refere-se aos parâmetros encontrados na ref.[30], RI da ref.[31] e LP refer-se aos valores que simulam os efeitos da interação de alcance finito.

$$
\begin{aligned}
P \mathcal{H} P= & \frac{1}{2 k_{F}^{2}}\left(k_{p}^{\prime 2}-k_{h}^{\prime 2}\right) \delta_{\vec{k}_{p} \vec{k}_{p}} \delta_{\vec{k}_{h}^{\prime} \vec{k}_{h}} \\
& +\frac{m}{\hbar^{2} k_{F}^{2}} \sum_{S_{a} T_{a}}\left(2 S_{a}+1\right)\left(2 T_{a}+1\right) W\left(\frac{1}{2} \frac{1}{2} \frac{1}{2} \frac{1}{2} ; S_{a} S\right) W\left(\frac{1}{2} \frac{1}{2} \frac{1}{2} \frac{1}{2} ; T_{a} T\right) \\
& \times \frac{C_{o}}{\mathcal{V}}\left\{F_{o}+2 F_{o}^{\prime}\left[T_{a}\left(T_{a}+1\right)-\frac{3}{2}\right]+2 G_{o}\left[S_{a}\left(S_{a}+1\right)-\frac{3}{2}\right]\right. \\
& \left.+4 G_{o}^{\prime}\left[S_{a}\left(S_{a}+1\right)-\frac{3}{2}\right]\left[T_{a}\left(T_{a}+1\right)-\frac{3}{2}\right]\right\}
\end{aligned}
$$

sendo $\mathcal{V}$ o volume da caixa e $W(a b c d ; e f)$ a função de Racah. No apêndice B escrevemos as expressões (B.4) e (B.5) de onde partimos para chegarmos no resultado acima. $O$ primeiro passo é calcular o bracket com a interação $V$ em (B.5) e com este resultado, reduzir os coeficientes de Clebsch-Gordan (de spin e isospin) em (B.4) e (B.5), na função de Racah.

Se desligarmos a interação, ficamos apenas com a primeira linha da equação acima que nos dá a resposta livre no gás de Fermi. 
II - Interação Matriz-G - O nosso modelo nuclear permite ainda o uso de uma interação de alcance finito que inclui termos de troca e forças tensoriais. A interação residual é uma matriz-G derivada do potencial de Bonn nucleon-nucleon de troca de méson. Aqui usamos a matriz-G da matéria nuclear de alcance finito da ref.[27]. A interação matriz-G antissimetrizada é,

$$
\begin{aligned}
G= & F+F^{\prime} \vec{\tau}_{1} \cdot \vec{\tau}_{2}+\bar{G} \vec{\sigma}_{1} \cdot \vec{\sigma}_{2}+\bar{G}^{\prime} \vec{\sigma}_{1} \cdot \vec{\sigma}_{2} \vec{\tau}_{1} \cdot \vec{\tau}_{2} \\
& +\imath \bar{S}\left(\vec{\sigma}_{1}+\vec{\sigma}_{2}\right) \cdot \hat{n}+\imath \bar{S}^{\prime}\left(\vec{\sigma}_{1}+\vec{\sigma}_{2}\right) \cdot \hat{n} \vec{\tau}_{1} \cdot \vec{\tau}_{2} \\
& +\bar{T} S_{12}(\hat{q})+\bar{T}^{\prime} S_{12}(\hat{q}) \vec{\tau}_{1} \cdot \vec{\tau}_{2}+\bar{H} S_{12}(\hat{Q})+\bar{H}^{\prime} S_{12}(\hat{Q}) \vec{\tau}_{1} \cdot \vec{\tau}_{2} .
\end{aligned}
$$

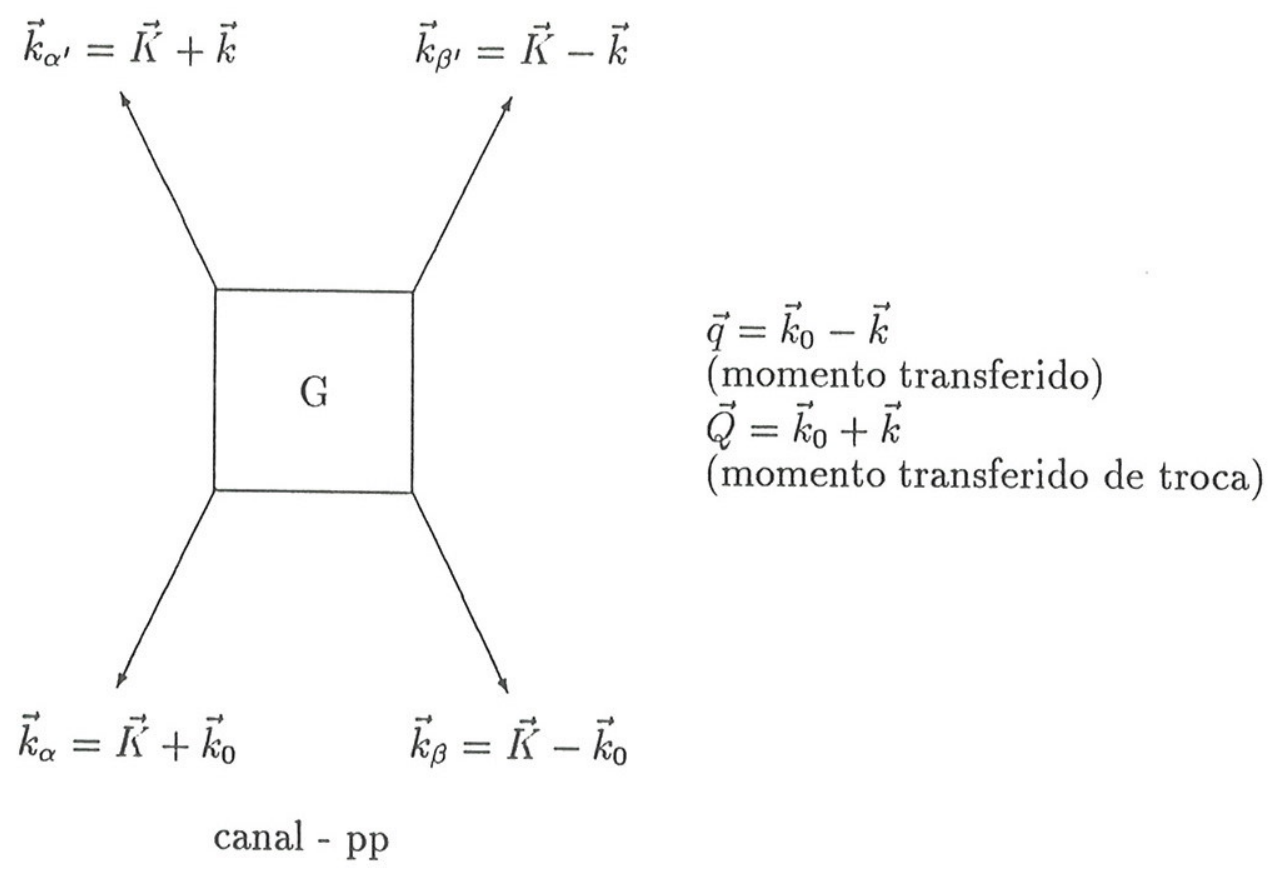

Figura 2.1: Representação diagramática da matriz-G.

Os coeficientes da interação central $F, F^{\prime}, \bar{G}, \bar{G}^{\prime}$, de spin-órbita $\bar{S}, \bar{S}^{\prime}$ e tensorial $\bar{T}, \bar{T}^{\prime}, \bar{H}, \bar{H}^{\prime}$ dependem da transferência de momento direto $\vec{q}$ e de troca $\vec{Q}$ e $\hat{n}$ é 
definido como $\hat{n}=\frac{\vec{q} \times \vec{Q}}{|\vec{q} \times \vec{Q}|}$. Os coeficientes são dados por

$$
\begin{array}{ll}
F=\frac{1}{16}\left\{3 G^{01}+G^{00}+3 G^{10}+9 G^{11}\right\}, & F^{\prime}=\frac{1}{16}\left\{G^{01}-G^{00}-3 G^{10}+3 G^{11}\right\}, \\
\bar{G}=\frac{1}{16}\left\{-3 G^{01}-G^{00}+G^{10}+3 G^{11}\right\}, & \bar{G}^{\prime}=\frac{1}{16}\left\{-G^{01}+G^{00}-G^{10}+G^{11}\right\}, \\
\bar{S}=\frac{1}{4}\left\{G_{L S}^{10}+3 G_{L S}^{11}\right\}, & \bar{S}^{\prime}=\frac{1}{4}\left\{-G_{L S}^{10}+G_{L S}^{11}\right\}, \\
\bar{T}=\frac{1}{4}\left\{G_{t}^{10}+3 G_{t}^{11}\right\}, & \bar{T}^{\prime}=\frac{1}{4}\left\{-G_{t}^{10}+G_{t}^{11}\right\}, \\
\bar{H}=\frac{1}{4}\left\{G_{t^{\prime}}^{10}+3 G_{t^{\prime}}^{11}\right\}, & \bar{H}^{\prime}=\frac{1}{4}\left\{-G_{t^{\prime}}^{10}+G_{t^{\prime}}^{11}\right\},
\end{array}
$$

onde os fatores $G^{m n}$ são

$$
\begin{array}{ll}
G^{00}=A^{S O}\left(\frac{m_{S O}^{2}}{q^{2}+m_{S O}^{2}}-\frac{m_{S O}^{2}}{Q^{2}+m_{S O}^{2}}\right), & G^{01}=A^{S E}\left(\frac{m_{S E}^{2}}{q^{2}+m_{S E}^{2}}+\frac{m_{S E}^{2}}{Q^{2}+m_{S E}^{2}}\right), \\
G^{10}=A^{T E}\left(\frac{m_{T E}^{2}}{q^{2}+m_{T E}^{2}}+\frac{m_{T E}^{2}}{Q^{2}+m_{T E}^{2}}\right), & G^{11}=A^{T O}\left(\frac{m_{T O}^{2}}{q^{2}+m_{T O}^{2}}-\frac{m_{T O}^{2}}{Q^{2}+m_{T O}^{2}}\right), \\
G_{L S}^{10}=\frac{1}{4} A^{L S E}\left(\frac{|\vec{q} \times \vec{Q}|}{q^{2}+m_{L S E}^{2}}-\frac{|\vec{q} \times \vec{Q}|}{Q^{2}+m_{L S E}^{2}}\right), & G_{L S}^{11}=\frac{1}{4} A^{L S O}\left(\frac{|\vec{q} \times \vec{Q}|}{q^{2}+m_{L S O}^{2}}+\frac{|\vec{q} \times \vec{Q}|}{Q^{2}+m_{L S O}^{2}}\right), \\
G_{t}^{10}=A^{T N E} \frac{|\vec{q}|^{2}}{q^{2}+m_{T N E}^{2}}\left(\frac{\Lambda_{T N E}^{2}-m_{T N E}^{2}}{\Lambda_{T N E}^{2}+q^{2}}\right), & G_{t^{\prime}}^{10}=A^{T N E} \frac{|\overrightarrow{\mid}|^{2}}{Q^{2}+m_{T N E}^{2}}\left(\frac{\Lambda_{T N E}^{2}-m_{T N E}^{2}}{\Lambda_{T N E}^{2}+Q^{2}}\right), \\
G_{t}^{11}=A^{T N O} \frac{|\vec{Q}|^{2}}{q^{2}+m_{T N O}^{2}}\left(\frac{\Lambda_{T N O}^{2}-m_{T N O}^{2}}{\Lambda_{T N O}^{2}+q^{2}}\right), & G_{t^{\prime}}^{11}=-A^{T N O} \frac{|\vec{Q}|^{2}}{Q^{2}+m_{T N O}^{2}}\left(\frac{\Lambda_{T N O}^{2}-m_{T N O}^{2}}{\Lambda_{T N O}^{2}+Q^{2}}\right) .
\end{array}
$$

$\Lambda_{i}$ é o cutoff do fator de forma $\left(\frac{\Lambda_{i}^{2}-m_{i}^{2}}{\Lambda_{i}^{2}+q^{2}}\right)$. Na tabela (2.2) encontram-se os valores para $\Lambda_{i}, m_{i}$ e $A^{i}$.

A interação efetiva como dada acima foi construída de modo que pudesse ser usada em cálculos de estrutura nuclear e é designada para energias na vizinhança da superfície de Fermi. A nossa escolha pela matriz-G se deve ao fato dela estar sendo muito usada em cálculos microscópicos[13][28]. 
Tabela 2.2: Parametrização da interação matriz-G baseada na versão HM3A.

\begin{tabular}{||l|r|r|r|r||}
\hline \hline & $m_{1}=4.0 \mathrm{fm}^{-1}$ & $m_{2}=2.5 \mathrm{fm}^{-1}$ & $m_{3}=0.71 \mathrm{fm}^{-1}$ & \\
\hline$S L$ & \multicolumn{1}{|c|}{$A$} & \multicolumn{1}{c|}{$A$} & $A$ & $\Lambda$ \\
\hline \hline$S O$ & 9324.6867 & -4498.1695 & 1195.3360 & \\
\hline$S E$ & 804.4224 & -1116.0621 & -398.4453 & \\
\hline$T O$ & 1897.4505 & -826.7796 & 132.8151 & \\
\hline$T E$ & 1883.2797 & -2375.8380 & -398.4453 & \\
\hline$T N O$ & -726.8203 & 392.2378 & -132.8151 & 960 \\
\hline$T N E$ & 4906.8850 & -1492.7610 & 398.4453 & 960 \\
\hline$L S O / 4.0$ & -12.6757 & -429.5220 & & \\
\hline$L S E / 4.0$ & 886.8376 & -162.3384 & & \\
\hline \hline
\end{tabular}

Três diferentes alcances $\left(m_{i}=1,3\right)$ são usados, sendo $m_{3}$ o potencial de troca de um píon. A intensidade $A$ de cada méson trocado está em unidades de $M e V \mathrm{fm}^{3}$. $\Lambda$ denota o cutoff no fator de forma e está em unidades de $\mathrm{MeV}$. A primeira coluna denota diferentes componentes da interação classificada de acordo com o spin $S$ (singleto ou tripleto) e momento angular $L$ (par ou ímpar) de estados de dois corpos. Para maiores detalhes veja refs. [27][29]. A versão HM3A[29] do potencial NN é usada.

Usando a interação acima, obtemos novamente uma expressão para a matriz $P \mathcal{H} P$,

$$
\begin{aligned}
P \mathcal{H} P & =\frac{1}{2 k_{F}^{2}}\left(k_{p}^{\prime 2}-k_{h}^{\prime 2}\right) \delta_{\vec{k}_{p} \vec{k}_{p}^{\prime}} \delta_{\vec{k}_{h}^{\prime} \vec{k}_{h}}+\frac{m}{\hbar^{2} k_{F}^{2}} \sum_{\substack{S_{a} M_{S_{a}} \\
M_{S_{b}}}} \sum_{m} \mathcal{C}_{P P}(-1)^{1-m_{s h}-m_{s h}^{\prime}} \\
& \times \sum_{T_{a}}\left(2 T_{a}+1\right) W\left(\frac{1}{2} \frac{1}{2} \frac{1}{2} \frac{1}{2} ; T_{a} T\right) \mathcal{F}\left(\vec{k}_{h}, \vec{k}_{p}^{\prime}, M_{S_{b}} ; \vec{k}_{p}, \vec{k}_{h}^{\prime}, S_{a}, T_{a}, M_{S_{a}}\right)
\end{aligned}
$$

onde

$$
\mathcal{C}_{P P} \equiv C_{m_{s p} m_{s h} M_{S}}^{\frac{1}{2}} \frac{1}{2} C_{m_{s p}^{\prime}}^{\frac{1}{2}} \underset{m_{s h}^{\prime} M_{S}}{\frac{1}{2}} C_{m_{s p}-m_{s h}^{\prime} M_{S_{a}}} C_{m_{s p}^{\prime}-m_{s h}}^{\frac{1}{2}} \frac{1}{2} M_{S_{b}}
$$

sendo $C_{\alpha \beta \gamma}^{a b c}$ o coeficiente de Clebsch-Gordan e $\sum_{m}$ significa uma soma sobre todas as projeções de spin da partícula e do buraco. Para obter o resultado acima também 
partimos das equações (B.4)e (B.5) do apêndice B. Como a interação matriz-G tem uma dependência com $M_{S_{a}}$ e $M_{S_{b}}$, não foi possível reduzir os coeficientes de ClebschGordan na função de Racah, como no caso anterior. Apenas a parte de isospin foi reduzida.

A solução de $\mathcal{F}$ é dada a seguir. Trocando $\mathcal{S}=\bar{S} /|\vec{q} \times \vec{Q}|, \mathcal{S}^{\prime}=\bar{S}^{\prime} /|\vec{q} \times \vec{Q}|$, $\mathcal{T}=\bar{T} /|\vec{q}|^{2}, \mathcal{T}^{\prime}=\bar{T}^{\prime} /|\vec{q}|^{2}, \mathcal{H}=\bar{H} /|\vec{Q}|^{2}$ e $\mathcal{H}^{\prime}=\bar{H}^{\prime} /|\vec{Q}|^{2}$, obtemos

$$
\mathcal{F}\left(\vec{k}_{h}, \vec{k}_{p}^{\prime}, M_{S_{b}} ; \vec{k}_{p}, \vec{k}_{h}^{\prime}, S_{a}, T_{a}, M_{S_{a}}\right)=\frac{1}{\mathcal{V}} \delta_{\vec{k}_{p}+\vec{k}_{h}^{\prime}, \vec{k}_{p}^{\prime}+\vec{k}_{h}}\left(\mathcal{F}_{\mathcal{R}}+\imath \mathcal{F}_{\mathcal{I}}\right)
$$

sendo

$$
\begin{aligned}
\mathcal{F}_{\mathcal{R}}= & \delta_{M_{S_{b}}, M_{S_{a}}}\left\{F+2 F^{\prime}\left[T_{a}\left(T_{a}+1\right)-\frac{3}{2}\right]+2 \bar{G}\left[S_{a}\left(S_{a}+1\right)-\frac{3}{2}\right]\right. \\
& +4 \bar{G}^{\prime}\left[T_{a}\left(T_{a}+1\right)-\frac{3}{2}\right]\left[S_{a}\left(S_{a}+1\right)-\frac{3}{2}\right] \\
& -\left(|\vec{q}|^{2} \mathcal{T}_{t}+|\vec{Q}|^{2} \mathcal{H}_{t}\right) 2 S_{a}\left(S_{a}+1\right)+\left(6 q_{z}^{2} \mathcal{T}_{t}+6 Q_{z}^{2} \mathcal{H}_{t}\right) M_{S_{a}}^{2} \\
& \left.+\left[3\left(q_{x}^{2}+q_{y}^{2}\right) \mathcal{T}_{t}+3\left(Q_{x}^{2}+Q_{y}^{2}\right) \mathcal{H}_{t}\right]\left[S_{a}\left(S_{a}+1\right)-M_{S_{a}}^{2}\right]\right\} \\
& +\delta_{M_{S_{b}}, M_{S_{a}}-1} \sqrt{S_{a}\left(S_{a}+1\right)-M_{S_{a}}\left(M_{S_{a}}-1\right)} \\
& \times\left[-\left(q_{z} Q_{x}-q_{x} Q_{z}\right) \mathcal{S}_{t}+\left(3 q_{x} q_{z} \mathcal{T}_{t}+3 Q_{x} Q_{z} \mathcal{H}_{t}\right)\left(2 M_{S_{a}}-1\right)\right] \\
& +\delta_{M_{S_{b}}, M_{S_{a}}+1} \sqrt{S_{a}\left(S_{a}+1\right)-M_{S_{a}}\left(M_{S_{a}}+1\right)} \\
& \times\left[\left(q_{z} Q_{x}-q_{x} Q_{z}\right) \mathcal{S}_{t}+\left(3 q_{x} q_{z} \mathcal{T}_{t}+3 Q_{x} Q_{z} \mathcal{H}_{t}\right)\left(2 M_{S_{a}}+1\right)\right] \\
& +\left(\delta_{M_{S_{b}}, M_{S_{a}}-2} S_{M}^{(-)}+\delta_{M_{S_{b}}, M_{S_{a}}+2} S_{M}^{(+)}\right)\left[\frac{3}{2}\left(q_{x}^{2}-q_{y}^{2}\right) \mathcal{T}_{t}+\frac{3}{2}\left(Q_{x}^{2}-Q_{y}^{2}\right) \mathcal{H}_{t}\right]
\end{aligned}
$$

e

$$
\begin{aligned}
\mathcal{F}_{\mathcal{I}}= & \delta_{M_{S_{b}}, M_{S_{a}}}\left(q_{x} Q_{y}-q_{y} Q_{x}\right) \mathcal{S}_{t} 2 M_{S_{a}} \\
& +\delta_{M_{S_{b}}, M_{S_{a}}-1} \sqrt{S_{a}\left(S_{a}+1\right)-M_{S_{a}}\left(M_{S_{a}}-1\right)}
\end{aligned}
$$




$$
\begin{aligned}
& \times\left[\left(q_{y} Q_{z}-q_{z} Q_{y}\right) \mathcal{S}_{t}+\left(3 q_{y} q_{z} \mathcal{T}_{t}+3 Q_{y} Q_{z} \mathcal{H}_{t}\right)\left(2 M_{S_{a}}-1\right)\right] \\
& +\delta_{M_{S_{b}}, M_{S_{a}}+1} \sqrt{S_{a}\left(S_{a}+1\right)-M_{S_{a}}\left(M_{S_{a}}+1\right)} \\
& \times\left[\left(q_{y} Q_{z}-q_{z} Q_{y}\right) \mathcal{S}_{t}-\left(3 q_{y} q_{z} \mathcal{T}_{t}+3 Q_{y} Q_{z} \mathcal{H}_{t}\right)\left(2 M_{S_{a}}+1\right)\right] \\
& +\left(\delta_{M_{S_{b}}, M_{S_{a}}-2} S_{M}^{(-)}-\delta_{M_{S_{b}}, M_{S_{a}}+2} S_{M}^{(+)}\right)\left(3 q_{x} q_{y} \mathcal{T}_{t}+3 Q_{x} Q_{y} \mathcal{H}_{t}\right)
\end{aligned}
$$

onde

$$
\begin{aligned}
\mathcal{T}_{t} & \equiv \mathcal{T}+2 \mathcal{T}^{\prime}\left[T_{a}\left(T_{a}+1\right)-\frac{3}{2}\right] \\
\mathcal{H}_{t} & \equiv \mathcal{H}+2 \mathcal{H}^{\prime}\left[T_{a}\left(T_{a}+1\right)-\frac{3}{2}\right] \\
\mathcal{S}_{t} & \equiv \mathcal{S}+2 \mathcal{S}^{\prime}\left[T_{a}\left(T_{a}+1\right)-\frac{3}{2}\right] \\
S_{M}^{( \pm)} & \equiv \sqrt{S_{a}\left(S_{a}+1\right)-M_{S_{a}}\left(M_{S_{a}} \pm 1\right)} \sqrt{S_{a}\left(S_{a}+1\right)-\left(M_{S_{a}} \pm 1\right)\left(M_{S_{a}} \pm 2\right)}
\end{aligned}
$$

$\operatorname{com} \vec{q}=\frac{\vec{k}_{p}-\vec{k}_{h}^{\prime}}{2}-\frac{\vec{k}_{h}-\vec{k}_{p}^{\prime}}{2}$ e $\vec{Q}=\frac{\vec{k}_{p}-\vec{k}_{h}^{\prime}}{2}+\frac{\vec{k}_{h}-\vec{k}_{p}^{\prime}}{2}$ em PHP. $\left(q_{x}, q_{y}, q_{z}\right)$ e $\left(Q_{x}, Q_{y}, Q_{z}\right)$ são as componentes de $\vec{q}$ e $\vec{Q}$, respectivamente.

\section{Detalhes numéricos e resultados}

Para dar início ao cálculo numérico, vamos considerar uma caixa com um volume $\mathcal{V}=1000 \mathrm{fm}^{3}(L=10 \mathrm{fm})$ e $k_{F}=1.36 \mathrm{fm}^{-1}$ que corresponde ao momento de Fermi da matéria nuclear infinita. Pode-se usar qualquer valor para o volume $\mathcal{V}$. Se $\mathcal{V}$ for muito pequeno teremos uma densidade de estados muito pequena ou até mesmo nenhum estado, e não seria entào possível fazer o cálculo de $R_{L} 1 \mathrm{p}-1 \mathrm{~h}$. se $\mathcal{V}$ for muito grande, teremos uma densidade de estados também muito grande e complicaria o calculo numérico. Assumimos o momento transferido na direção do eixo z. Limitaremos nossa discussão, neste trabalho, a valores relativamente elevados do momento transferido $\left(q^{(e)}>2 k_{F}\right)$. Em particular, consideramos $q^{(e)}=$ $550 \mathrm{MeV} / c$. Da equação $(2.33)$ temos que $\vec{k}_{p}=\vec{k}_{h}+\vec{q}^{(e)}$ e $\vec{k}_{p}^{\prime}=\vec{k}_{h}^{\prime}+\vec{q}^{(e)}$. Com 


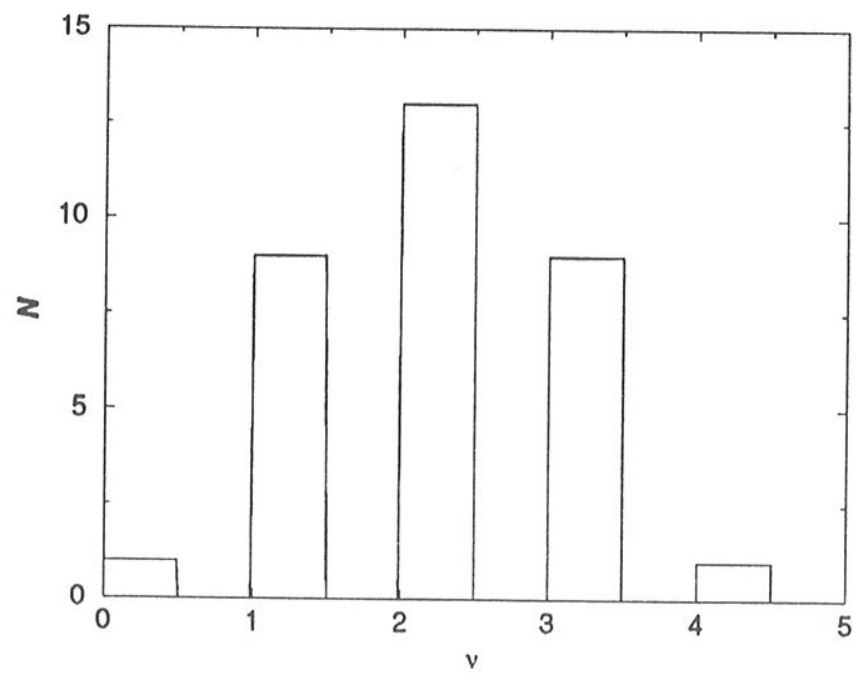

Figura 2.2: Histograma dos estados de partícula-buraco.

isto e a condição que a partícula deve estar acima do nível de Fermi e o buraco abaixo, podemos determinar os estados. Para facilitar o cálculo, podemos escrever os momentos da partícula e do buraco em termos de números quânticos. No caso do buraco,

$$
\begin{aligned}
& \vec{k}_{h}=\left(n_{h_{x}}, n_{h_{y}}, n_{h_{z}}\right) \frac{2 \pi}{L} ; \quad k_{h}<k_{F} \\
& \Longrightarrow \frac{4 \pi^{2}}{L^{2} k_{F}^{2}}\left(n_{h_{x}}^{2}+n_{h_{y}}^{2}+n_{h_{z}}^{2}\right)<1 .
\end{aligned}
$$

Com o volume considerado acima, cada $n$ varia de -2 a 2 . Encontramos então os estados do buraco e consequentemente da partícula. Com isto fica fácil obter a figura (2.2), que mostra um histograma, $N \times \nu$, dos estados da partícula e do buraco no espaço de p-h que entram no cálculo da função resposta (equação (2.32)). Vamos inicialmente calcular a resposta livre para mostrar o procedimento usado. 0 tipo de curva que obtemos do nosso cálculo é mostrado na figura (2.3). Observe que a posição $(\mathrm{em} \nu)$ e a altura dos picos estão intimamente ligados com a posição e o número de estados da figura (2.2). A largura dos picos vem do parâmetro $\eta$ da 


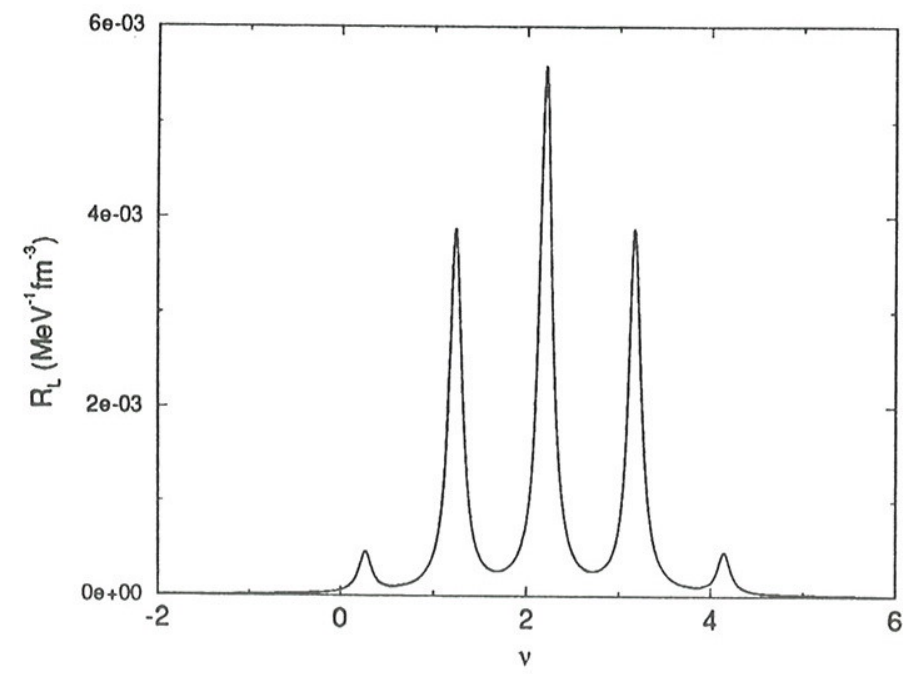

Figura 2.3: Resposta livre como obtida do cálculo da eq. (2.32).

equação (2.33). Aqui usamos o valor $\eta=0.08$. O passo seguinte é tomar médias dos pontos, ou seja, "alisar" a curva. O método usado é o "running average" (média corrente). O resultado disso é mostrado na figura (2.4) (curva tracejada). Para efeito ilustrativo mostramos, no mesmo gráfico, a resposta livre obtida através do cálculo analítico (curva sólida) por Fetter-Walecka[24]. A partir daqui não mostraremos mais os resultados numéricos do tipo da figura (2.3), mostraremos já as médias.

Seguindo o procedimento descrito acima podemos refazer o cálculo da resposta, mas mantendo agora as interações mencionadas anteriormente. Dessa forma, na figura (2.5) mostramos o cálculo numérico das equação (2.32) e (2.33) para diferentes valores dos parâmetros de Landau-Migdal e do parâmetro de intensidade $C_{o}$ (tabela (2.1)). A escolha dos parâmetros é de crucial importância e eles são dependentes do espaço de configuração e do modelo de partícula única que se usa. São ajustados para as propriedades de núcleo finito. Desse modo, vamos adotar duas parametrizações encontradas na literatura e calcular a resposta longitudinal p-h com elas. A curva pontilhada $(\mathrm{RK})$ refere-se a parametrização dada por Rinker e Speth[30] ${ }^{3}$. A curva

\footnotetext{
${ }^{3}$ Os parâmetros de Landau-Migdal foram ajustados a energias de excitação e momentos elétrico
} 


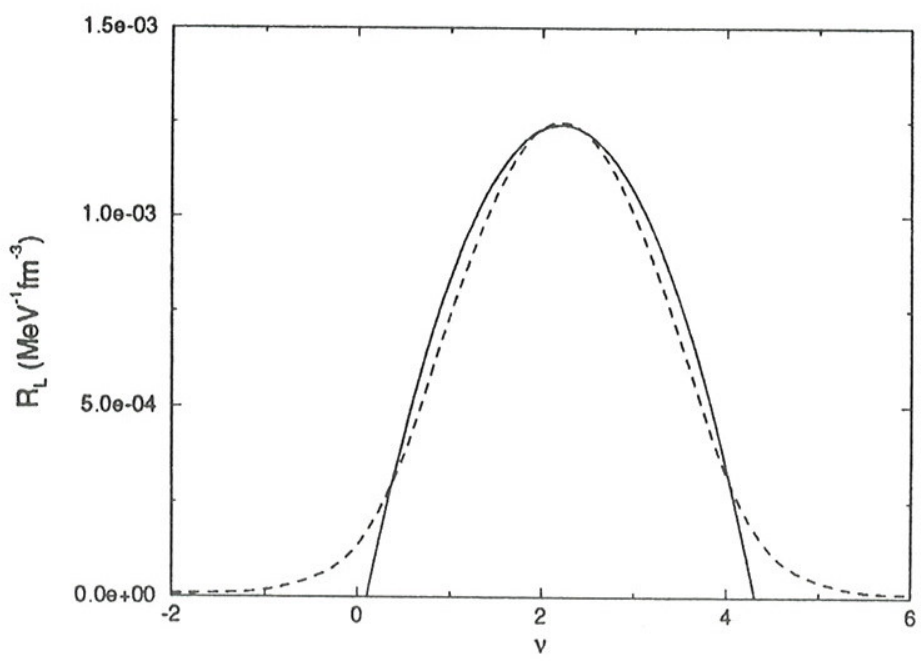

Figura 2.4: Resposta livre através de cálculo analítico (curva sólida) e através da eq. (2.32) (curva tracejada).

tracejada (RI) mostra a resposta p-h calculada com a parametrização dada por Ring e Speth[31 $]^{4}$. A curva sólida (HM3A) é o cálculo da resposta p-h usando a interação de alcance finito cujos coeficientes são dados na tabela (2.2).

A nossa análise dos resultados indica que os resultados obtidos com os parâmetros de Landau-Migdal (RK e RI), adotados neste trabalho, não apresentam uma diferença muito significativa em termos quantitativos e qualitativos. Porém, quando comparados com os resultados obtidos com a interação de alcance finito (HM3A), apresentam um caráter mais fortes.

Uma observação importante é que no espaço de p-h os efeitos da interação de alcance zero introduziu uma redução da intensidade, na região do pico da resposta livre, além de ter deslocado o pico para um $\nu$ maior. Contudo, a força de alcance zero, e magnético na região do chumbo $\left({ }^{204,206,207,208} \mathrm{~Pb}\right)$. A constante $C_{o}$ foi fixada para reproduzir o estado excitado coletivo mais baixo $3^{-}$a $6.13 \mathrm{MeV}$.

${ }^{4}$ Os parâmetros foram determinados por um fit dos momentos elétrico e magnético e razão de transição experimentalmente conhecidos dos núcleos ${ }^{207} \mathrm{~Pb},{ }^{209} \mathrm{~Pb},{ }^{207} \mathrm{Tl}$ e ${ }^{209} \mathrm{Bi}$ e a energia e tempo de vida dos estados mais baixos $3^{-}$e $2^{+}$no ${ }^{208} \mathrm{~Pb} . C_{o}=\frac{16}{9} \pi \epsilon_{F} r_{o}^{3}=380 \mathrm{MeV} \mathrm{fm} \mathrm{m}^{3}$, $r_{o}=1.2 \mathrm{fm}$ e $\epsilon_{F}=39.375 \mathrm{MeV}$. 


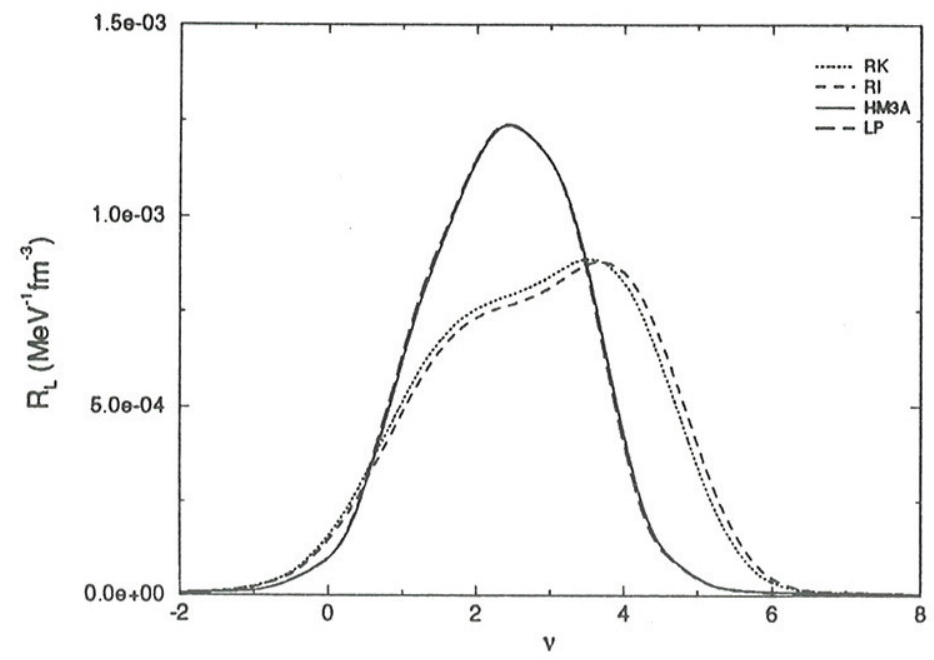

Figura 2.5: Resposta partícula-buraco com diferentes valores dos parâmetros de Landau-Migdal (RK, RI, LP) e com a interação matriz-G (HM3A).

que é constante no espaço de momento, é inapropriada para grandes transferências de momento. É perigoso usar esta força na descrição de processos para $q^{(e)}$ elevado, já que, geralmente, é empregada em fenômenos de baixa energia.

Com a interação de alcance finito notamos que a intensidade e a posição do pico, da resposta longitudinal no espaço de partícula-buraco, permanecem praticamente as mesmas em relação à resposta livre.

Uma vez que a interação de Landau-Migdal é mais simples de se trabalhar, obtemos os parâmetros $C_{o}, F_{o}$, etc através de um ajuste com a resposta $\mathrm{p}$-h obtida com a interação de alcance finito. A curva de traços longos (LP) mostra a resposta longitudinal p-h com esses novos valores dos parâmetros, que são denominados por LP na tabela (2.1). Observando mais atentamente a tabela (2.1) verificamos que os parâmetros LP são, na verdade, uma atenuação dos parâmetros RK por um fator de aproximadamente 4.

Embora os coeficientes da interação de alcance finito têm uma dependência com $\hat{q}$ e $\hat{Q}$, o mesmo não ocorrendo com a de alcance zero, conseguimos um excelente 
ajuste. Isto significa que, pelo menos ao nível de p-h, é possível descrever os efeitos da interação de alcance finito através de uma interação de alcance zero do tipo LandauMigdal, com parâmetros convenientemente escolhidos. Da tabela (2.1) podemos notar que esses novos valores dos parâmetros (LP) são os mesmos encontrados na primeira linha da tabela $(\mathrm{RK})$, diferem apenas na constante de acoplamento $C_{o}$. Por isso mesmo podem não mais reproduzir as propriedades físicas do núcleo finito, experimentalmente conhecidas, que foram usadas para obter os valores dos parâmetros $C_{o}, F_{o}$, etc de RK.

\subsubsection{Duas partículas-dois buracos - "SRPA"}

Nesta seção vamos introduzir os graus de liberdade de duas partículas-dois buracos. Assumimos agora uma base de operador de criação e destruição de estados p-h e $2 \mathrm{p}-2 \mathrm{~h}$, ou seja, $A_{i}=a_{h_{i}}^{\dagger} a_{p_{i}}+a_{h_{i 1}}^{\dagger} a_{h_{i 2}}^{\dagger} a_{p_{i 1}} a_{p_{i 2}}$. Usando novamente o método TDA e $|0\rangle=|H F\rangle$ e seguindo os mesmos passos que nos conduziram a função resposta p-h,

obtemos uma expressão que corresponde ao termo $\mathrm{O}^{\dagger} \mathcal{N} P \frac{1}{W_{P P}-W_{P Q} \frac{1}{W_{Q Q}} W_{Q P}} P \mathcal{N O}$ na equação (2.28).

Conseguimos, finalmente, a função resposta que é dada pela eq.(2.32) mas, neste caso, $M_{i j}^{I}$ contém os graus de liberdade $2 \mathrm{p}-2 \mathrm{~h}$ :

$$
\begin{aligned}
M_{i j}^{I}(\nu+\imath \eta) \equiv & C^{I} \sum_{\substack{k_{p}^{\prime}, k_{h}^{\prime} \\
k_{p}, k_{h}}} \delta_{\vec{k}_{p}^{\prime}, \vec{k}_{h}^{\prime}+\vec{q}^{(e)}} \theta\left(k_{p}^{\prime}-k_{F}\right) \theta\left(k_{F}-k_{h}^{\prime}\right) \\
& \times \theta\left(k_{1_{p}}^{\prime}-k_{F}\right) \theta\left(k_{2_{p}}^{\prime}-k_{F}\right) \theta\left(k_{F}-k_{1_{h}}^{\prime}\right) \theta\left(k_{F}-k_{2_{h}}^{\prime}\right) \\
& \times\left[\frac{1}{\nu+\imath \eta-P \mathcal{H} P-P \mathcal{H} Q \frac{1}{\nu+\imath \eta-Q \mathcal{H Q}} Q \mathcal{H P}}\right]_{\substack{k_{p}^{\prime} p_{h}^{\prime} \\
k_{p} k_{h}}}^{I} \\
& \times \delta_{\vec{k}_{p}, \vec{k}_{h}+\vec{q}^{(e)}} \theta\left(k_{p}-k_{F}\right) \theta\left(k_{F}-k_{h}\right)
\end{aligned}
$$




$$
\times \theta\left(k_{1_{p}}-k_{F}\right) \theta\left(k_{2_{p}}-k_{F}\right) \theta\left(k_{F}-k_{1_{h}}\right) \theta\left(k_{F}-k_{2_{h}}\right)
$$

onde

$$
C^{000}=C^{100}=\frac{Z}{2 N} F_{D}^{2}\left(q^{(e)}\right) .
$$

Para chegar no resultado acima usamos operadores eletromagnéticos[32] do tipo

$$
o^{I}\left(\vec{q}^{(e)}\right)=o^{I \dagger}\left(-\vec{q}^{(e)}\right)=F_{D}\left(q^{(e)}\right) \frac{g^{I}}{2} \sum_{i=1}^{A} O_{i}^{I}\left(\vec{q}^{(e)}\right) e^{\imath \vec{q}^{(e)} \cdot \vec{r}_{i}}
$$

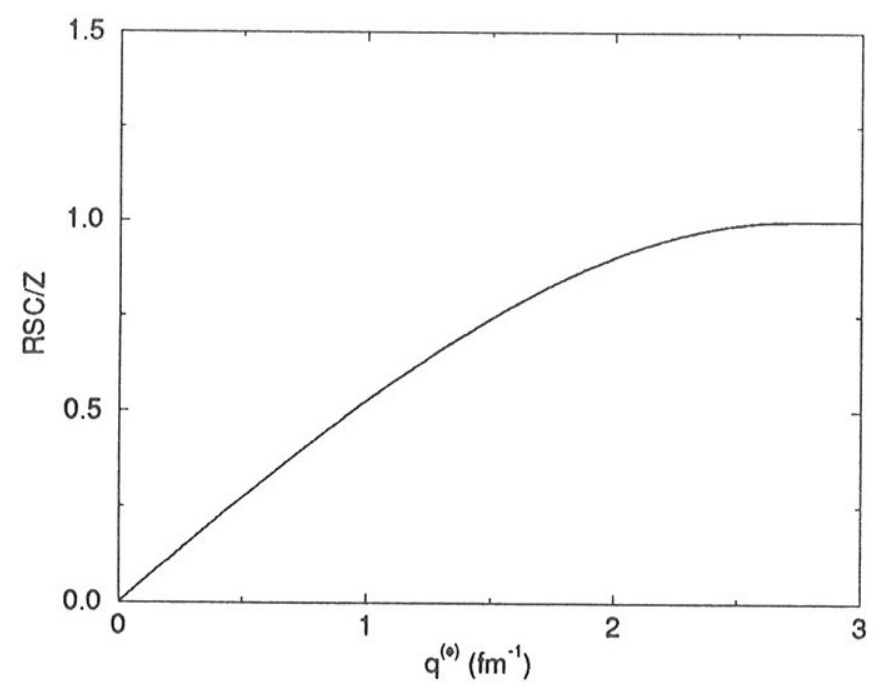

Figura 2.6: Regra de soma coulombiana dividida pelo número atômico, no modelo do Gás de Fermi para a resposta livre, como uma função de $q^{(e)}$ para $k_{F}=1.36 \mathrm{fm}^{-1}$. onde $F_{D}(q)=\left[1+\left(\frac{q \hbar c}{855 \mathrm{MeV}}\right)^{2}\right]^{-2}$ é o fator de forma eletromagnético $\gamma N N$ padrão. Quando a corrente de conveç̧ão é desprezada tem-se que $O^{000}=1, O^{100}=\tau_{3}(i)$ e $g^{000}=g^{100}=1$ para os operadores elétricos. No resultado acima trocamos $\tau_{3} \rightarrow \vec{\tau}$. O fator Z é obtido da regra de soma coulombiana (RSC) da resposta livre, no modelo do gás de Fermi. A figura (2.6) mostra $R S C / Z$ como uma função do momento 
transferido $q^{(e)}$ para $k_{F}=1.36 \mathrm{fm}^{-1}$. Neste caso, para $q^{(e)}=550 \mathrm{MeV} / \mathrm{c}$ temos que $Z=26$.

As matrizes $Q H P$ e $Q H Q$, na notação completa de spin e isospin, são dadas abaixo (veja apêndice $\mathrm{B})$ :

$$
\begin{gathered}
Q H P \equiv\left\langle\left[p_{j_{2}} h_{j_{2}}\right] S_{2}^{\prime} T_{2}^{\prime}\left[p_{j_{1}} h_{j_{1}}\right] S_{1}^{\prime} T_{1}^{\prime} ; S_{12}^{\prime} M_{S_{12}}^{\prime} T_{12}^{\prime} M_{T_{12}}^{\prime}|H|\left[p_{i} h_{i}\right] S M_{S} T M_{T}\right\rangle \\
Q H Q \equiv\left\langle\left[p_{i_{2}} h_{i_{2}}\right] S_{2} T_{2}\left[p_{i_{1}} h_{i_{1}}\right] S_{1} T_{1} ; S_{12} M_{S_{12}} T_{12} M_{T_{12}}\right| H \\
\left|\left[p_{j_{1}} h_{j_{1}}\right] S_{1}^{\prime} T_{1}^{\prime}\left[p_{j_{2}} h_{j_{2}}\right] S_{2}^{\prime} T_{2}^{\prime} ; S_{12}^{\prime} M_{S_{12}}^{\prime} T_{12}^{\prime} M_{T_{12}}^{\prime}\right\rangle
\end{gathered}
$$

A seguir mostramos as expressões obtidas usando a interação matriz-G como a interação residual:

$$
\begin{aligned}
Q \mathcal{H} P= & \frac{m}{\hbar^{2} k_{F}^{2}} \sum_{\substack{S_{a} M_{S_{a}} \\
M_{S_{b}}}} \sum_{M_{S_{1}}^{\prime} M_{S_{2}}^{\prime}} \sum_{m}(-1)^{\frac{3}{2}-m_{s 1 h}^{\prime}-m_{s 2 h}^{\prime}-m_{s h}} \mathcal{C}_{Q P} \\
& \times\left\{\mathcal{C}_{Q P}^{(a)} \delta_{m_{s 2 h}^{\prime} m_{s h}} \sum_{T_{a}}(-1)^{T_{1}^{\prime}-T_{2}^{\prime}+T} \mathcal{T}_{Q P} \delta_{T_{12}^{\prime} T} \delta_{M_{T_{12}}^{\prime} M_{T}} \mathcal{W}_{Q P}\right. \\
& \times 2 \mathcal{F}\left(\vec{k}_{1 p}^{\prime}, \vec{k}_{2 p}^{\prime}, M_{S_{b}} ; \vec{k}_{1 h}^{\prime}, \vec{k}_{p}, S_{a}, T_{a}, M_{S_{a}}\right) \delta_{\vec{k}_{h} \vec{k}_{2 h}^{\prime}} \\
& +\mathcal{C}_{Q P}^{(b)} \delta_{m_{s 2 p}^{\prime} m_{s p}} \sum_{T_{a}}(-1)^{T_{a}-1} \mathcal{T}_{Q P} \delta_{T_{12}^{\prime} T} \delta_{M_{T_{12}}^{\prime} M_{T}} \mathcal{W}_{Q P} \\
& \left.\times 2 \mathcal{F}\left(\vec{k}_{1 p}^{\prime}, \vec{k}_{h}, M_{S_{b}} ; \vec{k}_{2 h}^{\prime}, \vec{k}_{1 h}^{\prime}, S_{a}, T_{a}, M_{S_{a}}\right) \delta_{\vec{k}_{2 p}^{\prime} \vec{k}_{p}}\right\}
\end{aligned}
$$

com

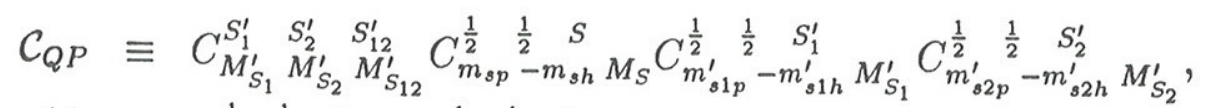

$$
\begin{aligned}
& \mathcal{C}_{Q P}^{(a)} \equiv C_{m_{s 1 h}^{\prime}}^{\frac{1}{2}} \stackrel{\frac{1}{2}}{m_{s p} M_{S_{a}}} C_{m_{s 2 p}^{\prime}}^{\frac{1}{2}} \stackrel{\frac{1}{2}}{m_{s 1 p}^{\prime} M_{S_{b}}} S_{a}
\end{aligned}
$$

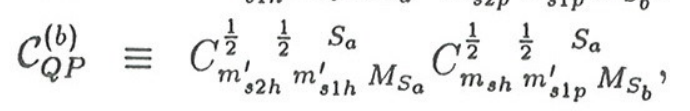




$$
\begin{aligned}
\mathcal{T}_{Q P} & \equiv\left(2 T_{a}+1\right)\left[\left(2 T_{1}^{\prime}+1\right)\left(2 T_{2}^{\prime}+1\right)\right]^{1 / 2} \\
\mathcal{W}_{Q P} & \equiv W\left(\frac{1}{2} \frac{1}{2} \frac{1}{2} \frac{1}{2} ; T_{a} T_{1}^{\prime}\right) W\left(\frac{1}{2} \frac{1}{2} T_{2}^{\prime} T_{1}^{\prime} ; T \frac{1}{2}\right) .
\end{aligned}
$$

A expressão da matriz $Q \mathcal{H} Q$ é

$$
\begin{aligned}
Q \mathcal{H} Q= & \frac{1}{2 k_{F}^{2}}\left(k_{1 p}^{2}+k_{2 p}^{2}-k_{1 h}^{2}-k_{2 h}^{2}\right) \delta_{\vec{k}_{1 p} \vec{k}_{1 p}^{\prime}} \delta_{\vec{k}_{2 p} \vec{k}_{2 p}^{\prime}} \delta_{\vec{k}_{1 h}^{\prime} \vec{k}_{1 h}} \delta_{\vec{k}_{2 h}^{\prime} \vec{k}_{2 h}} \delta_{S_{1} S_{1}^{\prime}} \delta_{S_{2} S_{2}^{\prime}} \delta_{T_{1} T_{1}^{\prime}} \delta_{T_{2} T_{2}^{\prime}} \\
& +\sum_{\substack{S_{a} M_{S_{a}} \\
M_{S_{b}}}} \sum_{\substack{M_{S_{S}} M_{S} \\
M_{S_{1}}^{\prime} M_{S_{2}}^{\prime 2}}}(-1)^{2-m_{s 1 h}^{\prime}-m_{s 2 h}^{\prime}-m_{s 1 h}-m_{s 2 h}} \\
& \times \mathcal{C}_{Q Q} \frac{m}{\hbar^{2} k_{F}^{2}}\left\{\mathcal{C}_{Q Q}^{(a)} \delta_{m_{s 1 h}} m_{s 1 h} \delta_{m_{s 2 h}^{\prime} m_{s 2 h}} \sum_{T_{a} f}(-1)^{T_{2}^{\prime}+T_{2}+T^{\prime}} \mathcal{T}_{Q Q} \mathcal{W}_{Q Q}\right. \\
& \times \mathcal{F}\left(\vec{k}_{1 p}, \vec{k}_{2 p}, M_{S_{b}} ; \vec{k}_{1 p}^{\prime}, \vec{k}_{2 p}^{\prime}, S_{a}, T_{a}, M_{S_{a}}\right) \delta_{\vec{k}_{1 h}^{\prime} \vec{k}_{1 h}} \delta_{\vec{k}_{2 h}^{\prime}} \vec{k}_{2 h} \\
& +\mathcal{C}_{Q Q}^{(b)} \delta_{m_{s 1 p} m_{s 1 p}^{\prime}} \delta_{m_{s 2 p} m_{s 2 p}^{\prime}} \sum_{T_{a} f}(-1)^{T_{1}^{\prime}+T_{1}+T^{\prime}} \mathcal{T}_{Q Q} \mathcal{W}_{Q Q} \\
& \times \mathcal{F}\left(\vec{k}_{1 h}^{\prime}, \vec{k}_{2 h}^{\prime}, M_{S_{b}} ; \vec{k}_{1 h}, \vec{k}_{2 h}, S_{a}, T_{a}, M_{S_{a}}\right) \delta_{\vec{k}_{1 p} \vec{k}_{1 p}^{\prime}} \delta_{\vec{k}_{2 p} \vec{k}_{2 p}^{\prime}} \\
& +\mathcal{C}_{Q Q}^{(c)} \delta_{m_{s 1 h} m_{s 1 h}^{\prime}} \delta_{m_{s 2 p} m_{s 2 p}^{\prime}} \sum_{T_{a} f}(-1)^{f+T^{\prime}-T_{a}+1} \mathcal{T}_{Q Q} \mathcal{W}_{Q Q} \\
& \times 2 \mathcal{F}\left(\vec{k}_{1 p}, \vec{k}_{2 h}^{\prime}, M_{S_{b}} ; \vec{k}_{2 h}, \vec{k}_{2 p}^{\prime}, S_{a}, T_{a}, M_{S_{a}}\right) \delta_{\vec{k}_{2 p} \vec{k}_{2 p}^{\prime}} \delta_{\vec{k}_{1 h}^{\prime} \vec{k}_{1 h}} \\
& +\mathcal{C}_{Q Q}^{(d)} \delta_{m_{s 2 p} m_{s 2 p}^{\prime}} \delta_{m_{s 2 h} m_{s 2 h}^{\prime}} \\
& \times \sum_{T_{a}}(-1)^{T_{1}^{\prime}+T_{1}}\left(2 T_{a}+1\right) W\left(\frac{1}{2} \frac{1}{2} \frac{1}{2} ; T_{1}^{\prime} T_{a}\right) \delta_{T_{1} T_{1}^{\prime}} \delta_{T_{2} T_{2}^{\prime}} \\
& \left.\times 2 \mathcal{F}\left(\vec{k}_{1 p}, \vec{k}_{1 h}^{\prime}, M_{S_{b}} ; \vec{k}_{1 h}, \vec{k}_{1 p}^{\prime}, S_{a}, T_{a}, M_{S_{a}}\right) \delta_{\vec{k}_{2 p} \vec{k}_{2 p}^{\prime}} \delta_{\vec{k}_{2 h}^{\prime} \vec{k}_{2 h}}\right\}
\end{aligned}
$$

onde usamos uma notação abreviada para vários coeficientes:

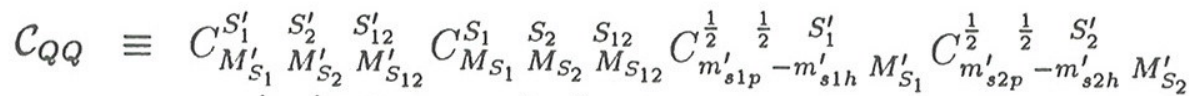

$$
\begin{aligned}
& \times C_{m_{s 1 p}-m_{s 1 h}}^{\frac{1}{2}} \underset{S_{1}}{\frac{1}{2}} M_{S_{1}} C_{m_{s 2 p}-m_{s 2 h}}^{\frac{1}{2}} \frac{1}{2} M_{S_{2}}
\end{aligned}
$$

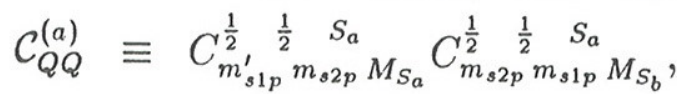




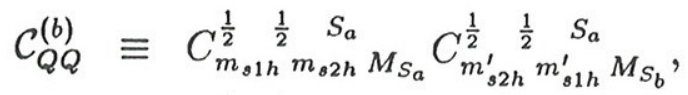

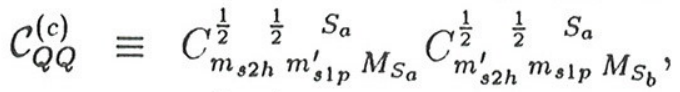

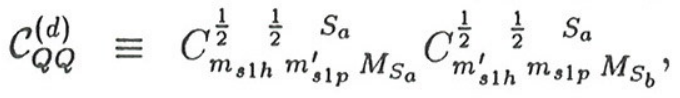

$$
\begin{aligned}
& \mathcal{T}_{Q Q} \equiv\left(2 T_{a}+1\right)(2 f+1)\left[\left(2 T_{1}+1\right)\left(2 T_{2}+1\right)\left(2 T_{1}^{\prime}+1\right)\left(2 T_{2}^{\prime}+1\right)\right]^{1 / 2}, \\
& \mathcal{W}_{Q Q} \equiv W\left(\frac{1}{2} \frac{1}{2} \frac{1}{2} \frac{1}{2} ; f T_{a}\right) W\left(T_{1} T_{1}^{\prime} \frac{1}{2} \frac{1}{2} ; f \frac{1}{2}\right) W\left(T_{2}^{\prime} T_{2} \frac{1}{2} \frac{1}{2} ; f \frac{1}{2}\right) W\left(T_{1}^{\prime} T_{1} T_{2}^{\prime} T_{2} ; f T\right) .
\end{aligned}
$$

A seguir mostramos as expressões para $Q \mathcal{H} P$ e $Q \mathcal{H} Q$, assumindo a interação de Landau-Migdal como a interação efetiva. Neste caso, conseguimos reduzir todos os coeficientes de Clebsch-Gordan na função de Racah. Encontramos os resultados,

$$
\begin{aligned}
Q \mathcal{H} P & =\sum_{S_{a} T_{a}}\left(2 S_{a}+1\right)\left[\left(2 S_{1}^{\prime}+1\right)\left(2 S_{2}^{\prime}+1\right)\right]^{1 / 2}\left(2 T_{a}+1\right)\left[\left(T_{1}^{\prime}+1\right)\left(2 T_{2}^{\prime}+1\right)\right]^{1 / 2} \\
& \times W\left(\frac{1}{2} \frac{1}{2} \frac{1}{2} \frac{1}{2} ; S_{1}^{\prime} S_{a}\right) W\left(\frac{1}{2} \frac{1}{2} S_{1}^{\prime} S_{2}^{\prime} ; S \frac{1}{2}\right) \delta_{S_{12}^{\prime} S} \delta_{M_{S_{12}}^{\prime} M_{S}} \\
& \times W\left(\frac{1}{2} \frac{1}{2} \frac{1}{2} \frac{1}{2} T_{1}^{\prime} T_{a}\right) W\left(\frac{1}{2} \frac{1}{2} T_{1}^{\prime} T_{2}^{\prime} ; T \frac{1}{2}\right) \delta_{T_{12}^{\prime} T} \delta_{M_{T_{12}}^{\prime} M_{T}} \\
& \times\left[(-1)^{S_{2}^{\prime}-S_{1}^{\prime}+S+T_{2}^{\prime}-T_{1}^{\prime}+T} \delta_{\vec{k}_{h} \vec{k}_{2 h}^{\prime}} \delta_{\vec{k}_{1 h}^{\prime}}+\vec{k}_{p}, \vec{k}_{1 p}^{\prime}+\vec{k}_{2 p}^{\prime}\right. \\
& \left.\times(-1)^{S_{a}+T_{a}} \delta_{\vec{k}_{2 p}^{\prime}} \vec{k}_{p} \delta_{\left.\vec{k}_{2 h}^{\prime}+\vec{k}_{1 h}^{\prime}, \vec{k}_{1 p}^{\prime}+\vec{k}_{h}\right]}\right] \\
& \frac{2 C_{o}}{\mathcal{V}}\left\{F_{o}+2 F_{o}^{\prime}\left[T_{a}\left(T_{a}+1\right)-\frac{3}{2}\right]+2 G_{o}\left[S_{a}\left(S_{a}+1\right)-\frac{3}{2}\right]\right. \\
& \left.+4 G_{o}^{\prime}\left[S_{a}\left(S_{a}+1\right)-\frac{3}{2}\right]\left[T_{a}\left(T_{a}+1\right)-\frac{3}{2}\right]\right\},
\end{aligned}
$$

$\mathrm{e}$

$$
\begin{aligned}
Q \mathcal{H} Q & =\frac{1}{2 k_{F}^{2}}\left(k_{1 p}^{2}+k_{2 p}^{2}-k_{1 h}^{2}-k_{2 h}^{2}\right) \delta_{\vec{k}_{1 p} \vec{k}_{1 p}^{\prime}} \delta_{\vec{k}_{2 p} \vec{k}_{2 p}^{\prime}} \delta_{\vec{k}_{1 h}^{\prime}} \vec{k}_{1 h} \delta_{\vec{k}_{2 h}^{\prime} \vec{k}_{2 h}} \delta_{S_{1} S_{1}^{\prime}} \delta_{S_{2} S_{2}^{\prime}} \delta_{T_{1} T_{1}^{\prime}} \delta_{T_{2} T_{2}^{\prime}} \\
+ & +\left[\sum_{f S_{a}}\left(2 S_{a}+1\right)(2 f+1)\left[\left(2 S_{1}^{\prime}+1\right)\left(2 S_{2}^{\prime}+1\right)\left(2 S_{1}+1\right)\left(2 S_{2}+1\right)\right]^{1 / 2}\right. \\
& \times W\left(S_{1}^{\prime} S_{1} S_{2}^{\prime} S_{2} ; f S\right) W\left(S_{1} S_{1}^{\prime} \frac{1}{2} \frac{1}{2} ; f \frac{1}{2}\right) W\left(S_{2}^{\prime} S_{2} \frac{1}{2} \frac{1}{2} ; f \frac{1}{2}\right) W\left(\frac{1}{2} \frac{1}{2} \frac{1}{2} \frac{1}{2} ; f S_{a}\right) \\
& \times \sum_{f^{\prime} T_{a}}\left(2 T_{a}+1\right)\left(2 f^{\prime}+1\right)\left[\left(2 T_{1}^{\prime}+1\right)\left(2 T_{2}^{\prime}+1\right)\left(2 T_{1}+1\right)\left(2 T_{2}+1\right)\right]^{1 / 2}
\end{aligned}
$$




$$
\begin{aligned}
& \times W\left(T_{1}^{\prime} T_{1} S_{2}^{\prime} T_{2} ; f^{\prime} T\right) W\left(T_{1} T_{1}^{\prime} \frac{1}{2} \frac{1}{2} ; f^{\prime} \frac{1}{2}\right) W\left(T_{2}^{\prime} T_{2} \frac{1}{2} \frac{1}{2} ; f^{\prime} \frac{1}{2}\right) W\left(\frac{1}{2} \frac{1}{2} \frac{1}{2} \frac{1}{2} ; f^{\prime} T_{a}\right) \\
& \times\left((-1)^{S_{2}^{\prime}+S_{2}+S+T_{2}^{\prime}+T_{2}+T} \delta_{\vec{k}_{1 h}^{\prime}} \vec{k}_{1 h} \delta_{\vec{k}_{2 h}^{\prime}} \vec{k}_{2 h} \delta_{\vec{k}_{1 p}^{\prime}+\vec{k}_{2 p}^{\prime}, \vec{k}_{1 p}+\vec{k}_{2 p}}\right. \\
& +(-1)^{S_{1}^{\prime}+S_{1}+S+T_{1}^{\prime}+T_{1}+T} \delta_{\vec{k}_{1 p} \vec{k}_{1 p}^{\prime}} \delta_{\vec{k}_{1 h}^{\prime}} \vec{k}_{1 h} \delta_{\vec{k}_{1 h}+\vec{k}_{2 h}, \vec{k}_{1 h}^{\prime}+\vec{k}_{2 h}^{\prime}} \\
& \left.\left.+(-1)^{f+S+1-S_{a}+f^{\prime}+T+1-T_{a}} \delta_{\vec{k}_{2 p} \vec{k}_{2 p}^{\prime}} \delta_{\vec{k}_{1 h}^{\prime}} \vec{k}_{1 h} \delta_{\vec{k}_{2 h}+\vec{k}_{1 p}^{\prime}, \vec{k}_{1 p}+\vec{k}_{2 h}^{\prime}}\right)\right] \\
& +\sum_{S_{a}}(-1)^{S_{1}+S_{1}^{\prime}}\left(2 S_{a}+1\right) W\left(\frac{1}{2} \frac{1}{2} \frac{1}{2} \frac{1}{2} ; S S_{a}\right) \delta_{S_{1} S_{1}^{\prime}} \delta_{S_{2} S_{2}^{\prime}} \\
& \times \sum_{T_{a}}(-1)^{T_{1}+T_{1}^{\prime}}\left(2 T_{a}+1\right) W\left(\frac{1}{2} \frac{1}{2} \frac{1}{2} \frac{1}{2} ; T T_{a}\right) \delta_{T_{1} T_{1}^{\prime}} \delta_{T_{2} T_{2}^{\prime}} \\
& \left.\times 2 \delta_{\vec{k}_{2 p} \vec{k}_{2 p}^{\prime}} \delta_{\vec{k}_{2 h}^{\prime} \vec{k}_{2 h}} \delta_{\vec{k}_{1 h}+\vec{k}_{1 p}^{\prime}, \vec{k}_{1 p}+\vec{k}_{1 h}^{\prime}}\right\} \\
& \times \frac{C_{o}}{\mathcal{V}}\left\{F_{o}+2 F_{o}^{\prime}\left[T_{a}\left(T_{a}+1\right)-\frac{3}{2}\right]+2 G_{o}\left[S_{a}\left(S_{a}+1\right)-\frac{3}{2}\right]\right. \\
& \left.+4 G_{o}^{\prime}\left[S_{a}^{\prime}\left(S_{a}+1\right)-\frac{3}{2}\right]\left[T_{a}\left(T_{a}+1\right)-\frac{3}{2}\right]\right\} .
\end{aligned}
$$

\section{Detalhes numéricos e resultados}

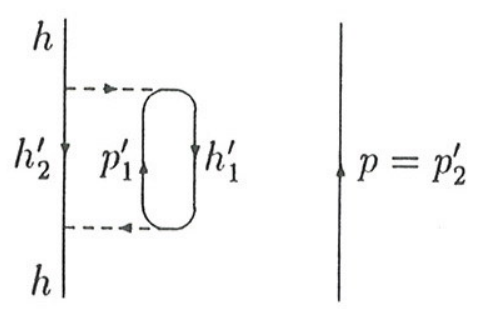

(a) autoenergia do buraco

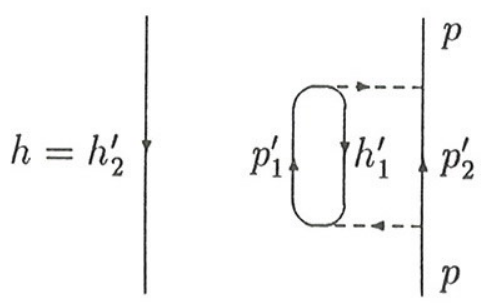

(b) autoenergia da partícula

Figura 2.7: Representação diagramática das autoenergias.

É uma tarefa difícil resolver um problema de autovalor que trata explicitamente os graus de liberdade $2 \mathrm{p}$ - $2 \mathrm{~h}$ com a mesma simplicidade como os de $\mathrm{p}$-h. Isto porque o número de estados $2 \mathrm{p}-2 \mathrm{~h}(\mathrm{n})$ é tão grande que não se pode tratar explicitamente 
estes graus de liberdade. Veja, por exemplo, as figuras (2.8) e (2.9) que mostram $n \times \nu$ para a autoenergia do buraco (figura (2.7a)) e da partícula (figura (2.7b)), respectivamente, no canal $I \equiv\{000\}$. Para o canal $I \equiv\{100\}$, as figuras (2.10) e (2.11) mostram resultados semelhantes. No apêndice $C$ escrevemos as expressões para o cálculo do número total de estados para as autoenergias da partícula e do buraco.

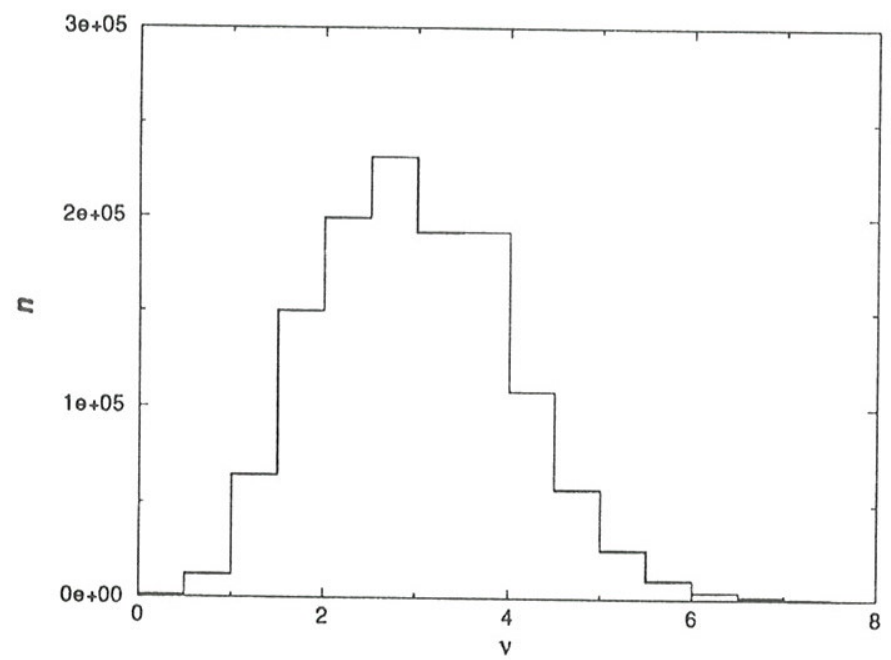

Figura 2.8: Histograma dos estados duas partículas-dois buracos da autoenergia do buraco no canal $I \equiv\{000\}$.

A saída que encontramos para evitar esta dificuldade é considerar uma amostra destes estados que represente adequadamente bem todos eles, em cada $\nu$ (figuras (2.12) e (2.13)), ou seja, vamos escolher aleatóriamente um número menor de estados, obtidos nas figuras (2.8), (2.9), (2.10) e (2.11) mas que mantenham a mesma distribuição. Podemos então trabalhar com matrizes menores, viáveis em nosso cálculo numérico. Para restaurar a contribuição de todos os estados $2 \mathrm{p}-2 \mathrm{~h}$ devemos multiplicar o termo $P \mathcal{H} Q \frac{1}{\nu+\imath \eta-Q \mathcal{H} Q} Q \mathcal{H} P$ da equação (2.47), por um fator que é dado por: 


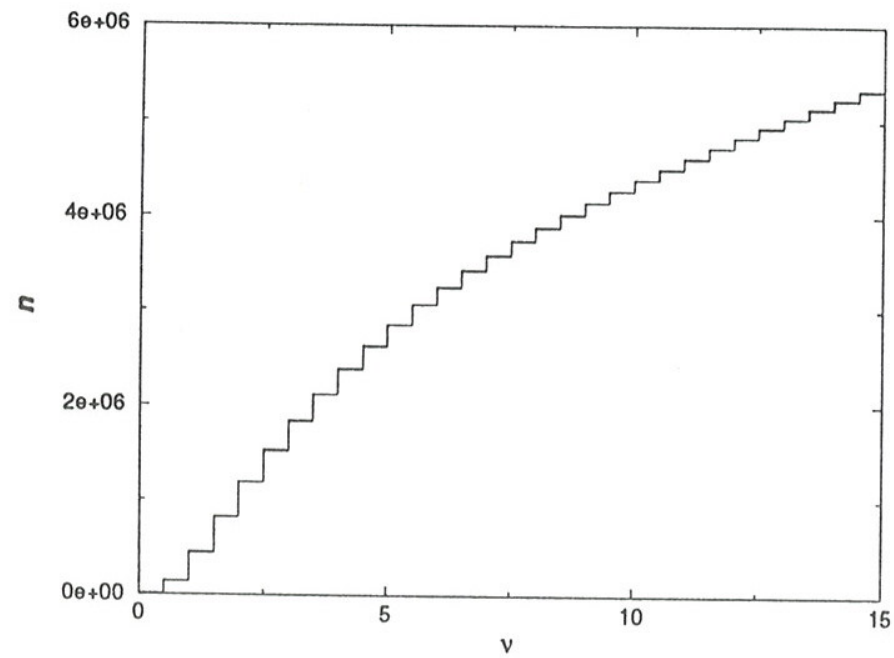

Figura 2.9: Histograma dos estados duas partículas-dois buracos da autoenergia da partícula no canal $I \equiv\{000\}$.

$$
\frac{n}{m}=\frac{n^{\circ} \text { total de estados } \mathrm{h}(\mathrm{p})}{n^{\circ} \text { de estados da amostra } \mathrm{h}(\mathrm{p})}
$$

Devemos ainda lembrar que podemos encontrar estados da partícula em qualquer valor de $\nu$. Como pode ser visto nos gráficos, optamos por fazer um corte em $\nu=15$ $(\omega \simeq 1151 \mathrm{MeV})$.

No gráfico (2.14) mostramos o cálculo das equações (2.32) e (2.47) sem a interação p-h, ou seja, calculamos a resposta livre (curva sólida). Os estados são aqueles da figura (2.2). Seguimos o mesmo procedimento, descrito na seção anterior, de "alisar" a curva. Mostramos também a resposta longitudinal obtida experimentalmente (quadrados) do ${ }^{56} \mathrm{Fe}[4]$ com as barras de erro.

Com os estados, cujas características são mostradas nas figuras (2.12) e (2.13), prosseguimos para o cálculo numérico da resposta longitudinal no espaço de $2 \mathrm{p}-2 \mathrm{~h}$, usando as equações $P \mathcal{H} P, Q \mathcal{H} P$ e $Q \mathcal{H} Q$ obtidas com a interação de alcance finito. A curva sólida na figura (2.15) mostra esse resultado e sugere que, quando trata- 


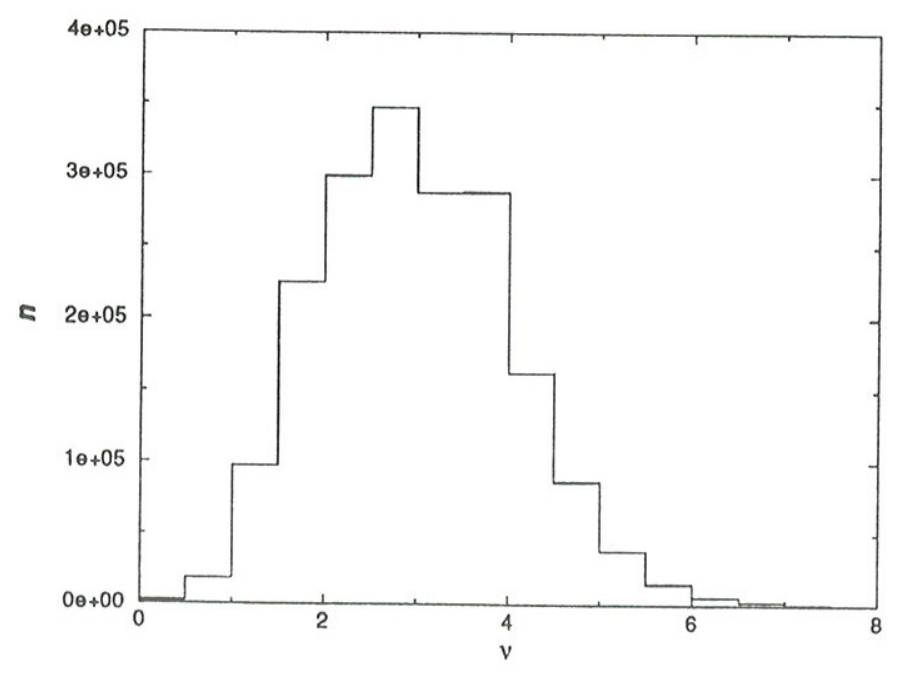

Figura 2.10: Histograma dos estados duas partículas-dois buracos da autoenergia do buraco no canal $I \equiv\{100\}$.

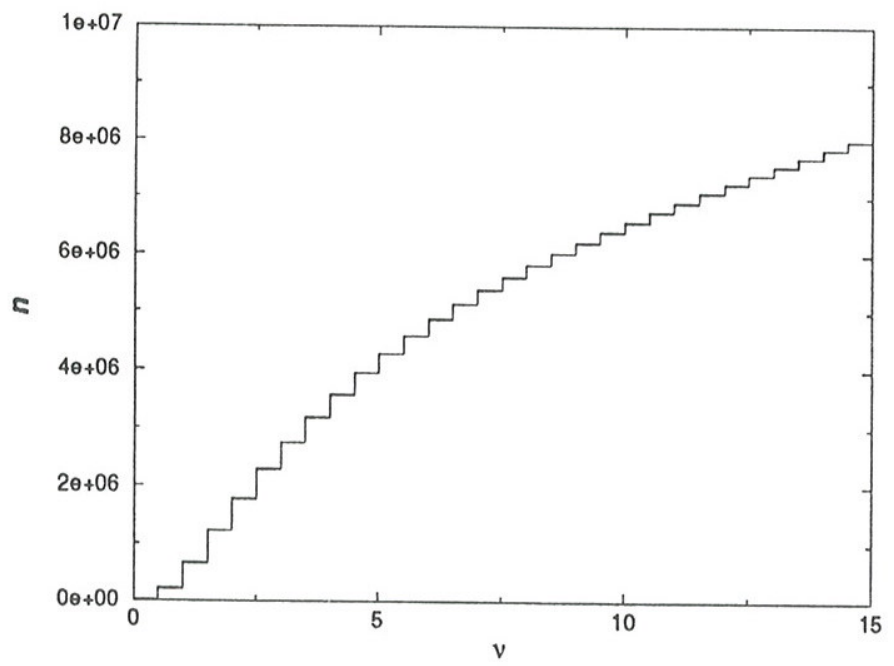

Figura 2.11: Histograma dos estados duas partículas-dois buracos da autoenergia da partícula no canal $I \equiv\{100\}$. 


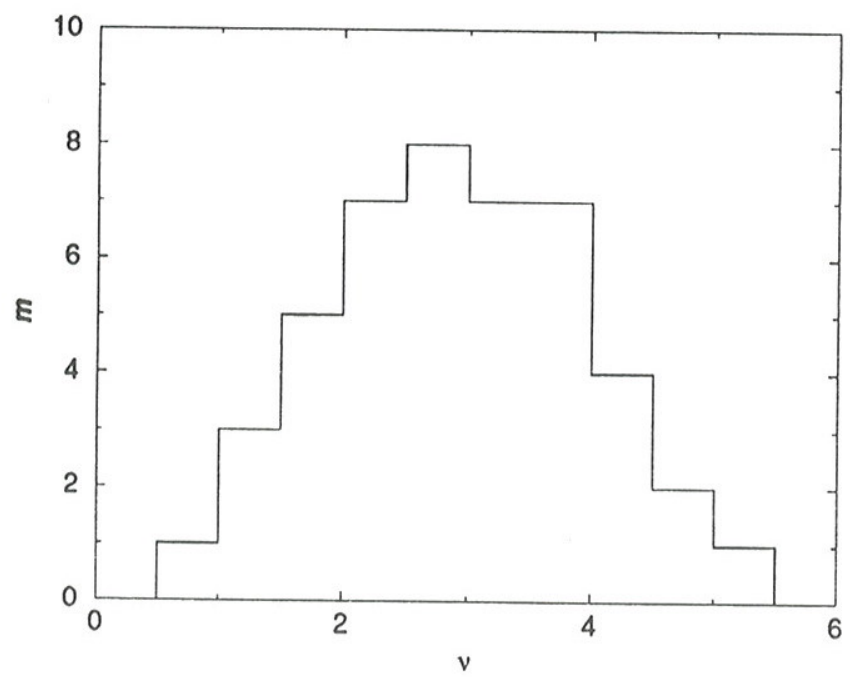

Figura 2.12: Histograma da amostra dos estados duas partículas-dois buracos da autoenergia do buraco.

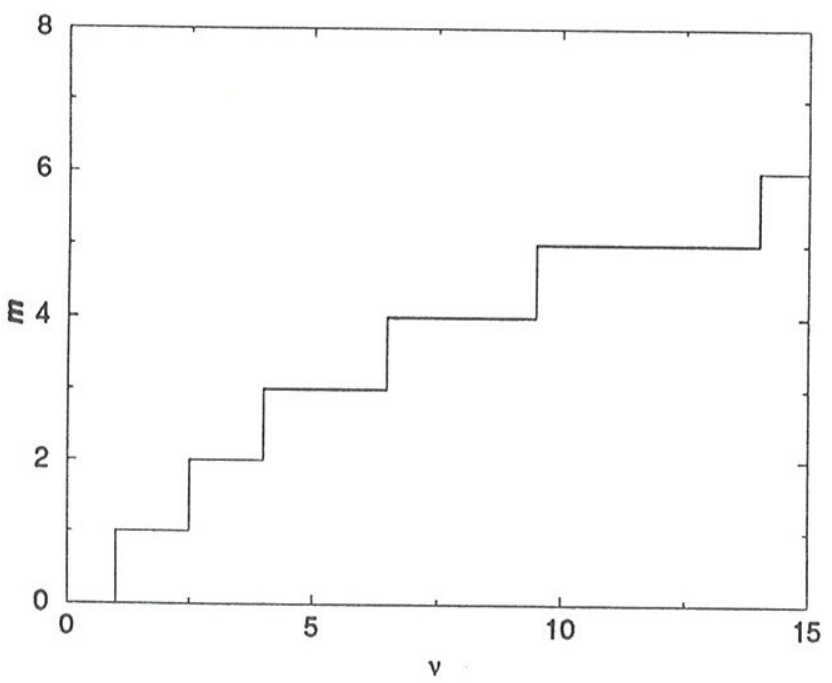

Figura 2.13: Histograma da amostra dos estados duas partículas-dois buracos da autoenergia da partícula. 


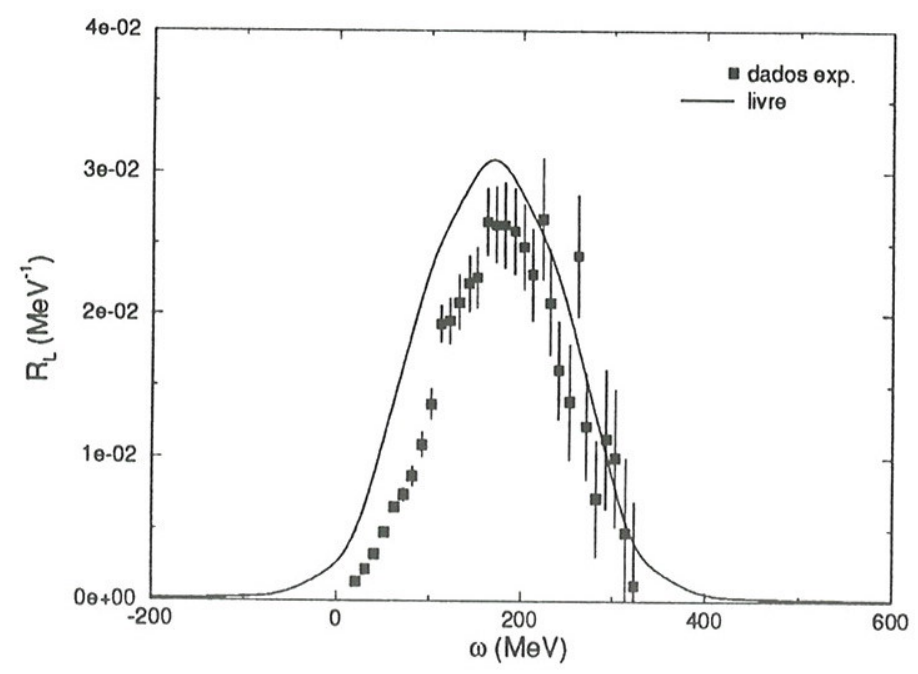

Figura 2.14: Resultado do cálculo das equações (2.32) e (2.47) sem a interação p-h (curva sólida) e dados experimentais do ${ }^{56} \mathrm{Fe}$.

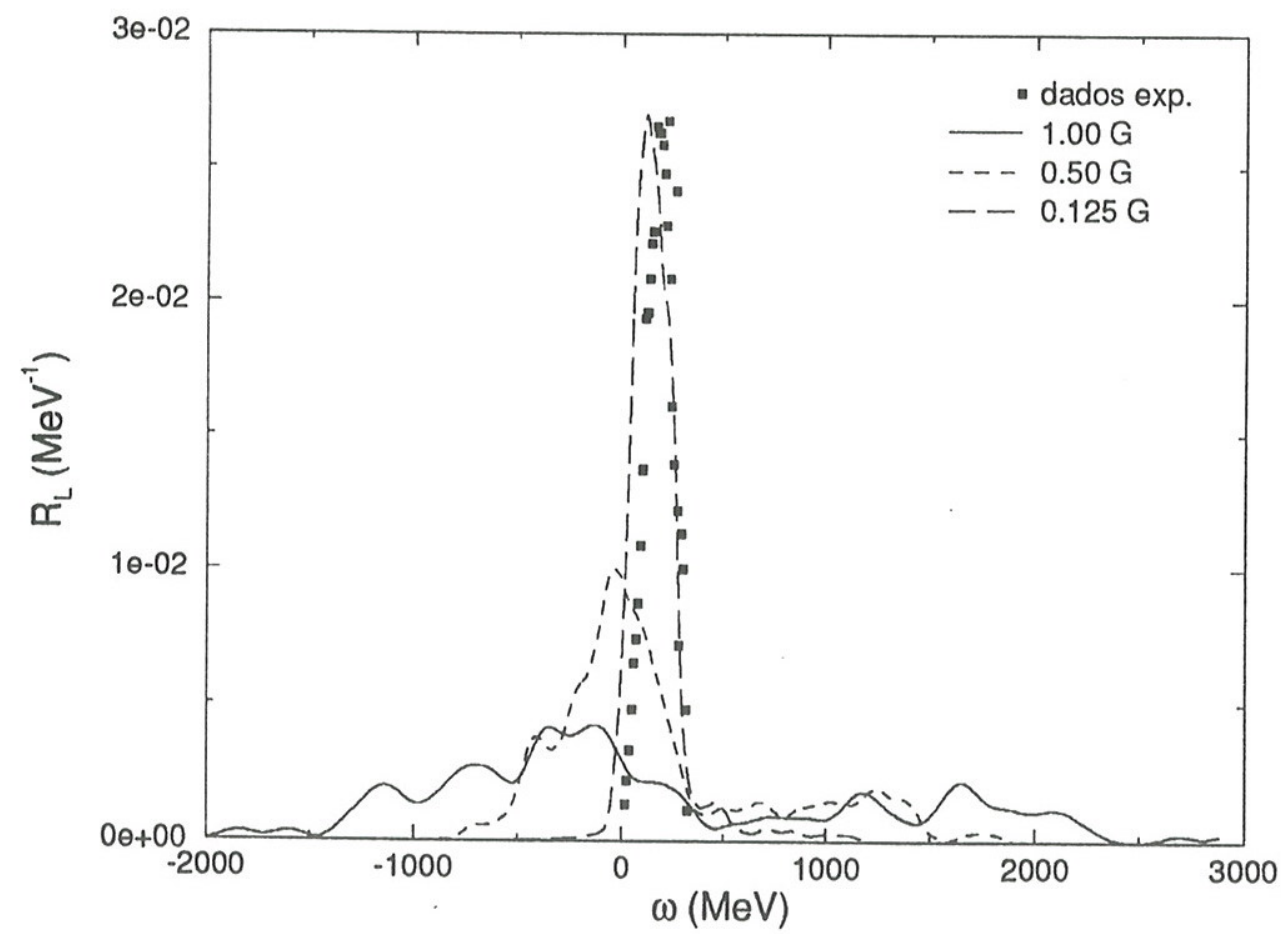

Figura 2.15: Resposta longitudinal no espaço de $2 \mathrm{p}-2 \mathrm{~h}$ para diferentes atenuação da interação matriz-G. 
mos com 2p-2h, a interação matriz-G apresenta um comportamento muito forte a ponto de espalhar a curva por um alcance maior de $\omega$, além de diminuir consideravelmente a intensidade na região do pico e desloca-lo para energias mais baixas, quando comparada com a resposta livre. Ao atenuarmos a interação (curva tracejada, $0.50 G$, e curva de traços longos, $0.125 G$ ) aproximamos nossos resultados aos dados experimentais do ${ }^{56} \mathrm{Fe}$ (quadrados, sem as barras de erro). Podemos ainda notar que temos resposta $2 \mathrm{p}-2 \mathrm{~h}$ para valores negativos da energia. Isto vem da própria inconsistência do modelo: na verdade, o que explica esse comportamento é que há estados correlacionados com energia menor que a do modelo adotado para o estado fundamental. Na figura (2.16) mostramos mais alguns resultados da resposta longitudinal 2p-2h para outras atenuações da interação.

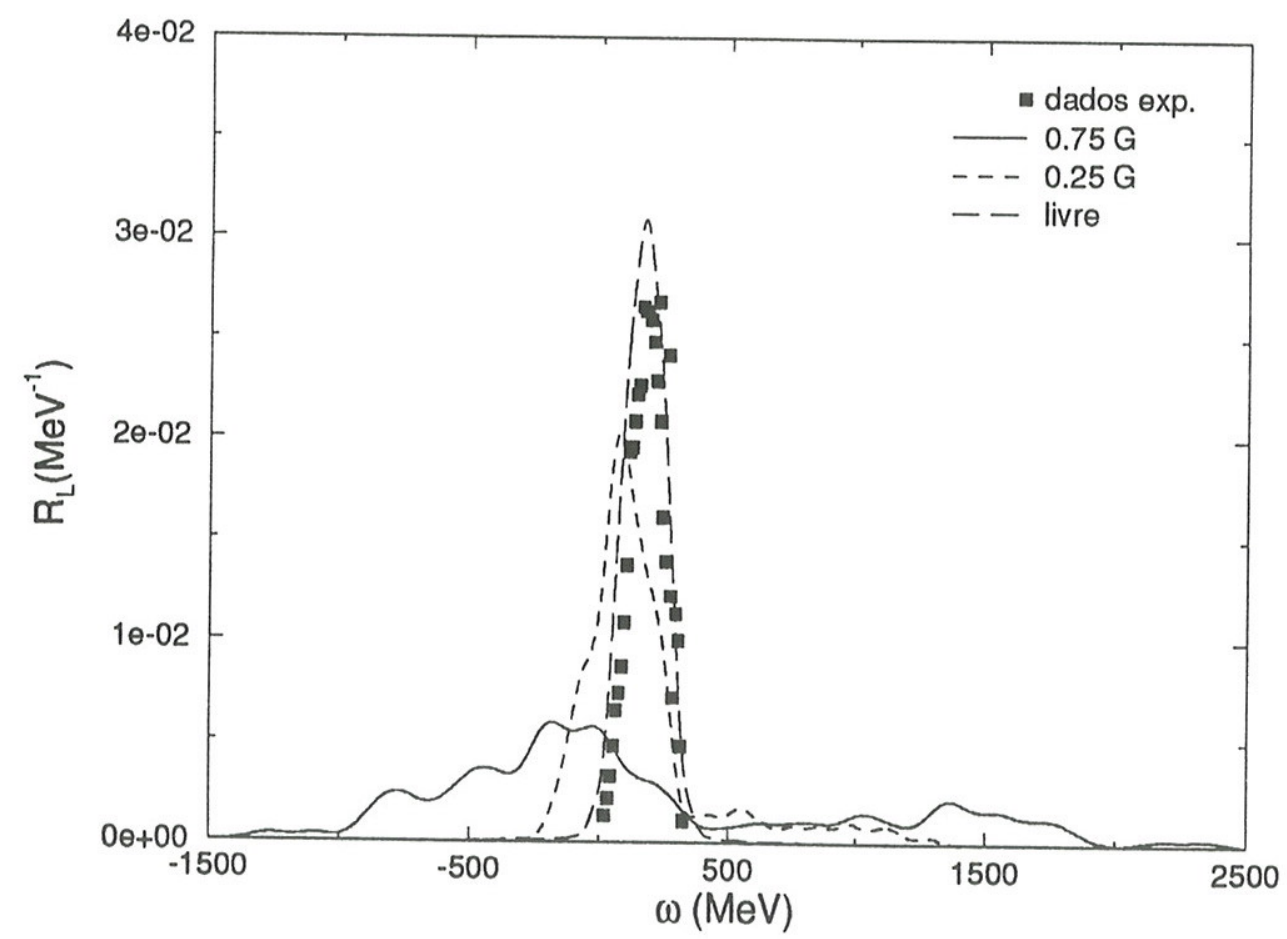

Figura 2.16: Resposta longitudinal 2p-2h com duas atenuações da interação MatrizG. 
Uma análise dos resultados nos mostra que a curva não cai rapidamente a zero como na resposta $\mathrm{p}$-h a medida que $\omega$ aumenta. Possui um valor baixo, porém aproximadamente uniforme, por um longo alcance da energia. Isto vem dos estados da autoenergia da partícula (figura (2.7b)). Isto porque, nesta região de energia, temos apenas estados da autoenergia da partícula. O alcance diminui a medida que aumentamos a atenuação da interação. Contudo, mesmo para a maior atenuação $(0.125 G)$, temos resposta longitudinal até o valor de $\omega$ onde encontramos estados da partícula $(\omega \simeq 1151 \mathrm{MeV})$. À medida que diminuimos a atenuação, a intensidade da curva, nessa região de energia, aumenta e se espalha por um alcance maior de $\omega$.

Este é um resultado muito interessante pois pode explicar o "quenching" da regra de soma. Recorrendo à regra de soma coulombiana[9],

$$
R S C=\int \frac{R_{L}\left(q^{(e)}, \omega\right)}{F_{D}^{2}\left(q^{(e)}\right)} d \omega
$$

podemos obter a carga atômica contida somente no pico principal das curvas mostradas nas figuras (2.15) e (2.16). Na tabela (2.3) registramos esses valores lembrando que integramos a equação $(2.57)$ até $\omega=350 \mathrm{MeV}$.

Tabela 2.3: Resultados da regra de soma coulombiana para o ${ }^{56} \mathrm{Fe}$.

\begin{tabular}{||c|c||c|c||}
\hline \hline & $R S C$ & $C\left(q^{(e)}\right)$ & $\frac{C\left(q^{(e)}\right)}{F_{D}^{2}\left(q^{(e)}\right)}$ \\
\hline \hline $0.125 \mathrm{G}$ & 24.2135 & $4.6 \pm 1.07$ & $18.4 \pm 4.28$ \\
\hline $0.250 \mathrm{G}$ & 22.3180 & & \\
\hline $0.500 \mathrm{G}$ & 19.7865 & & \\
\hline $0.750 \mathrm{G}$ & 18.2588 & & \\
\hline $1.000 \mathrm{G}$ & 17.2936 & & \\
\hline \hline
\end{tabular}

Podemos observar que o valor da carga atômica cresce a medida que aumentamos a atenuação da interação. Entretanto, a regra de soma não é saturada quando con- 
sideramos apenas o pico principal da curva. A intensidade que falta está justamente na região de $\omega$ acima de $350 \mathrm{MeV}$ e onde não temos dados experimentais confiáveis da resposta longitudinal.

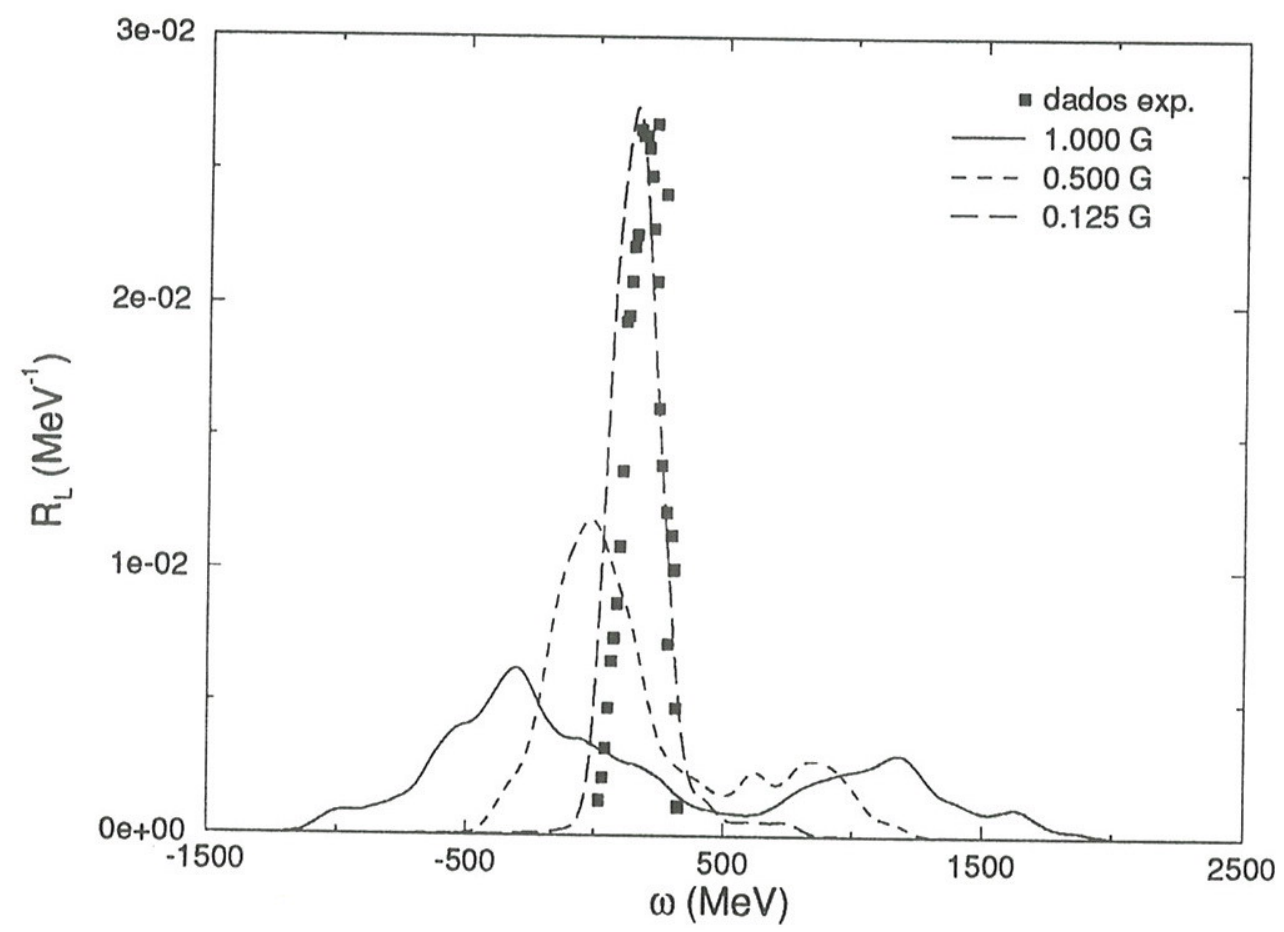

Figura 2.17: Resposta longitudinal 2p-2h com atenuações da interação matriz-G com estados até $\nu=10$.

$\mathrm{Na}$ ref. [4] encontramos um resultado da regra de soma, para o ${ }^{56} \mathrm{Fe}$ à $q^{(e)}=$ $550 \mathrm{MeV} / \mathrm{c}$, calculada a partir dos dados experimentais da resposta longitudinal que são mostrados também na figura (2.14). A soma coulombiana é calculada através da expressão

$$
C\left(q^{(e)}\right)=\int_{0^{+}}^{\omega_{\max }} R_{L}\left(\vec{q}^{(e)}, \omega\right) d \omega .
$$

onde o limite $0^{+}$exclui espalhamento elástico e $\omega_{\max }$ é o limite experimental além do qual os dados não são confiáveis. Para $\omega_{\max }=350 \mathrm{MeV}$ o valor encontrado 
de $C\left(q^{(e)}\right)$ está registrado na tabela (2.3). Observe que a equação acima difere da equação (2.57) pelo quadrado do fator de forma do nucleon $F_{D}^{2}\left(q^{(e)}\right)$. Neste caso, vamos dividir o valor de $C\left(q^{(e)}\right)$ pelo quadrado do fator de forma usado em nossos cálculos. O resultado é mostrado na quarta coluna da tabela (2.3). Na figura (2.17) mostramos a resposta longitudinal no espaço $2 \mathrm{p}-2 \mathrm{~h}$ calculada considerando os estados obtidos até $\nu=10$. Apresenta o mesmo comportamento das figuras anteriores

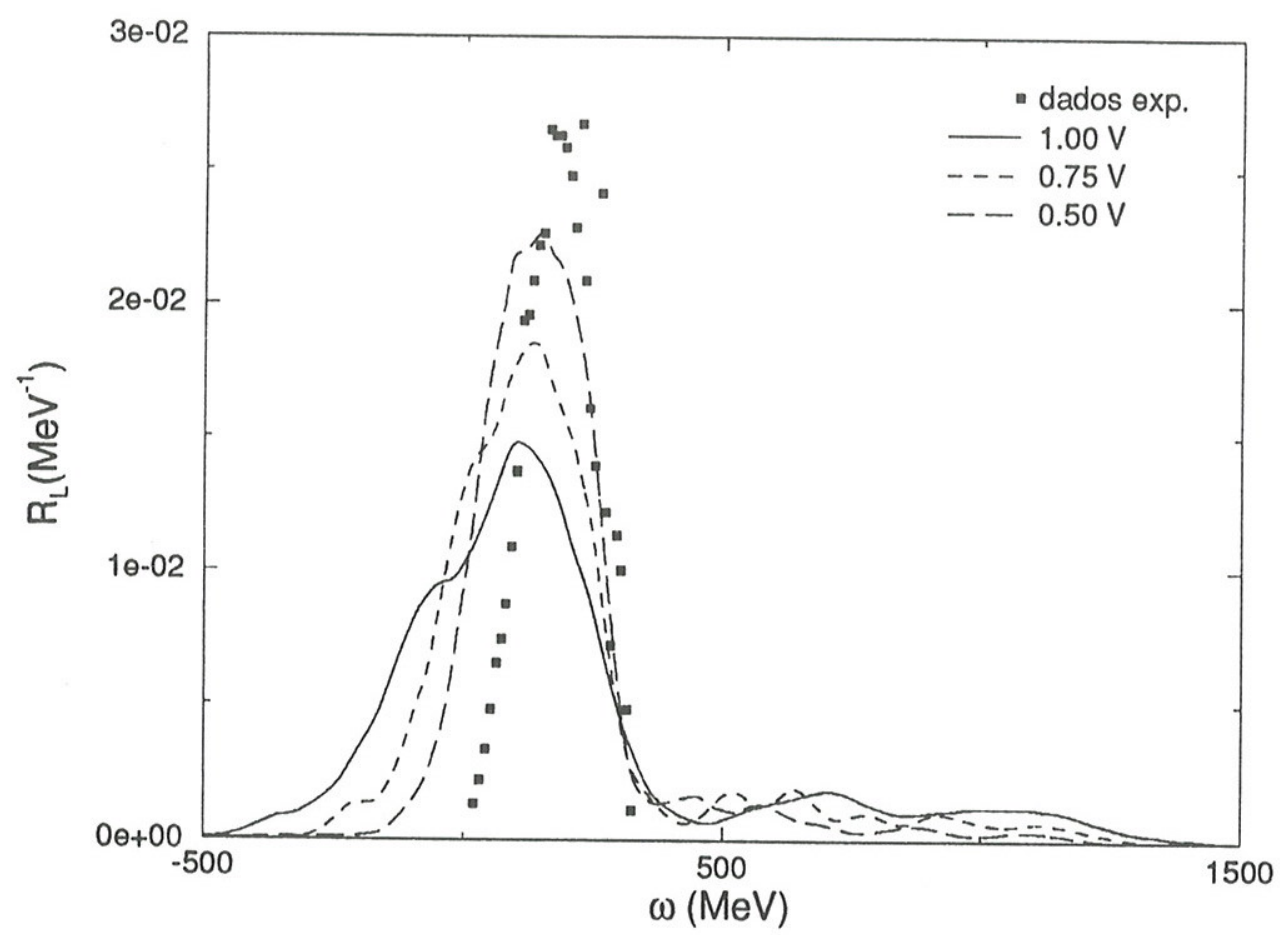

Figura 2.18: Resposta longitudinal 2p-2h com atenuações da interação do tipo Landau-Migdal.

Além da resposta longitudinal $2 \mathrm{p}-2 \mathrm{~h}$ considerando todos os elementos da matriz $Q \mathcal{H} Q$, como é calculada neste trabalho, algumas vezes é feito um tratamento diagonal dessa matriz. Isto é, considera-se na matriz apenas os elementos da diagonal. Comparamos resultados numéricos obtidos nos dois tratamentos citados logo acima e 
nenhuma diferença significativa foi verificada, tanto em termos quantitativos quanto qualitativos.

Calculamos também a resposta longitudinal no espaço $2 \mathrm{p}-2 \mathrm{~h}$ com uma interação do tipo Landau-Migdal. Na figura (2.18) mostramos esse resultado usando os parâmetros denominados LP. Observamos que a resposta apresenta as mesmas características daquela obtida com a interação de alcance finito, embora o fator de atenuação não seja o mesmo. De qualquer forma, nos dois casos, precisamos atenuar a interação para aproximarmos os resultados aos dados experimentais. 


\section{Capítulo 3}

\section{Tratamento das Respostas Eletromagnéticas em Termos de uma Teoria de Reações}

Neste capítulo, apresentamos um formalismo que permite obter expressões para seções de choque inclusiva ou exclusiva usando a técnica da teoria de reações de Feshbach e a representação óptica de fundo. Podemos separar a seção de choque de espalhamento em duas contribuições incoerentes: uma contribuição "óptica" ou lisa e uma contribuição de flutuação. Usamos o método de Kawai, Kerman e McVoy[21] (KKM) para escrevermos a seção de choque de flutuação. A objetivo final é exprimir a seção de choque de flutuação em termos de potenciais ópticos através do formalismo desenvolvido por Kerman e McVoy[23] (KM), no contexto do espalhamento inelástico de elétrons. 


\subsection{Formalismo}

A reação que vamos estudar é uma reação sequencial ou de dois passos do tipo $A\left(e, e^{\prime}\right) B+C$, sendo $C$ qualquer coisa e $B$ pode ser, por exemplo, um proton. Neste caso temos a situação em que um eletron $e$ interage com um núcleo $A$ e se afasta deixando-o num estado excitado $A^{*}$ (reação direta), que depois decai sequencialmente num estado $C$ emitindo um próton $p$.

$$
\begin{aligned}
e+A \longrightarrow e^{\prime}+A^{*} & \\
& \longrightarrow C+p .
\end{aligned}
$$

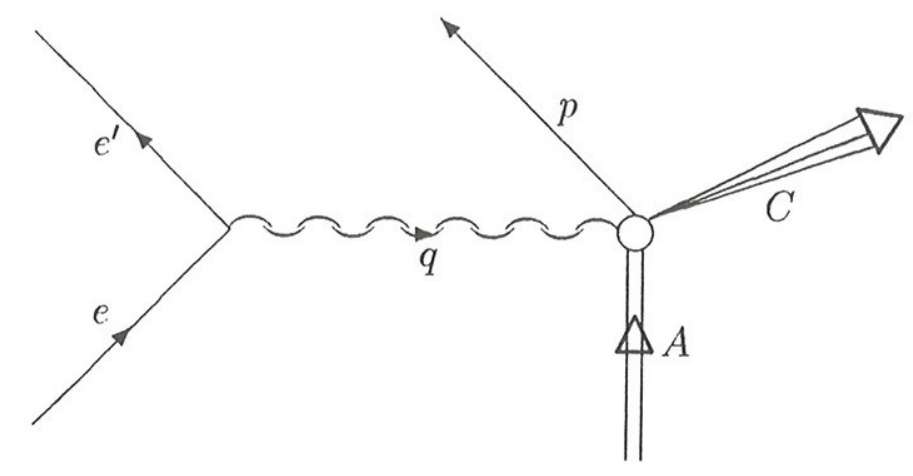

Figura 3.1: Representação diagramática do processo de espalhamento do elétron.

A amplitude de transição para esta reação pode ser escrita como

$$
T_{f i}=\left\langle\Phi_{f}^{(-)} u_{e^{\prime}}^{(-)}\left|H_{i n t}\right| \Psi_{e, i}^{(+)}\right\rangle .
$$

Assumimos que o elétron é tratado na aproximação de Born com ondas distorcidas (DWBA) aproximando $\Psi_{e, i}^{(+)}$por $\Phi_{i} u_{e}^{(+)}$e conseguimos 


$$
\begin{aligned}
T_{f i} & =\left\langle\Phi_{f}^{(-)}\left|\mathcal{O}_{e e^{\prime}}\right| \Phi_{i}\right\rangle, \\
\mathcal{O}_{e e^{\prime}} & =\left\langle u_{e^{\prime}}^{(-)}\left|H_{i n t}\right| u_{e}^{(+)}\right\rangle
\end{aligned}
$$

onde

- $\Phi_{f}^{(-)}$é o estado do núcleo residual,

- $u_{e^{\prime}}^{(-)}$corresponde ao estado final do elétron,

- $\Psi_{e, i}^{(+)}$é o estado do sistema inicial,

- $u_{e}^{(+)}$corresponde ao estado inicial do elétron,

- $\Phi_{i}$ é o estado inicial do alvo,

- $\mathcal{O}_{e e^{\prime}}$ é um operador de transição de um corpo, neste caso.

Vamos analizar isto com a teoria de reações de Feshbach. O ponto principal é decompor a função de onda do núcleo residual nas componentes relevantes para o estudo do nosso problema. Isto significa dividir o espaço de Hilbert associado ao núcleo residual em dois subespaços mutuamente acoplados que contenham informação relativa

a) às excitações de 1 partícula - 1 buraco $\left(\psi_{\sigma \tau}^{\dagger}\left(\overrightarrow{r^{\prime}}\right) \psi_{\sigma \tau}(\vec{r})\left|\phi_{i}\right\rangle\right)$ mais quaisquer canais abertos relevantes - subespaço $P$,

b) ao resto, isto é, as informações que não estão contidas no anterior $(2 \mathrm{p}-2 \mathrm{~b}, 3 \mathrm{p}-3 \mathrm{~b}$, núcleo composto $(\mathrm{NC}))$ - subespaço $Q=1-P(Q$ é ortogonal à $P)$.

Vamos colocar a energia do estado inicial igual a zero de modo que 


$$
H\left|\Phi_{i}\right\rangle=0
$$

e a equação de Schroedinger para o estado final como

$$
(E-H)\left|\Phi_{f}^{(+)}\right\rangle=0 .
$$

Assumindo $\mathcal{O}_{e e^{\prime}}$ ser um operador de um corpo podemos trabalhar com

$$
T_{f i} \simeq\left\langle\Phi_{f}^{(-)}\left|P \mathcal{O}_{e e^{\prime}}\right| \Phi_{i}\right\rangle .
$$

Para chegarmos aos resultados desejados precisamos de algumas ferramentas padrão:

- equações acopladas para $P \Phi_{f}$ e $Q \Phi_{f}$,

- hamiltoniana efetiva para $P \Phi_{f}$,

- representação óptica de fundo.

\subsubsection{Equações acopladas para $P \Phi_{f}$ e $Q \Phi_{f}$}

Usando os operadores de projeção $P$ e $Q$ (veja equação (2.27)) podemos escrever $\Phi_{f}^{(-)}$como

$$
\Phi_{f}^{(-)}=P \Phi_{f}^{(-)}+Q \Phi_{f}^{(-)},
$$

e também reescrever a equação de Schroedinger como duas equações acopladas

$$
\begin{aligned}
& \left(E-H_{P P}\right) P \Phi_{f}=H_{P Q} Q \Phi_{f} \\
& \left(E-H_{Q Q}\right) Q \Phi_{f}=H_{Q P} P \Phi_{f} .
\end{aligned}
$$


Omitimos nas equações acima o sinal (-) de $\Phi_{f}$. Colocaremos novamente no resultado final.

Nas equações acima temos que $H_{P P}=P H P, H_{Q Q}=Q H Q, H_{P Q}=P H Q$ e $H_{Q P}=Q H P$. Estes termos podem ser interpretados da seguinte forma:

a) $H_{P P}$ é o acoplamento do subespaço $P$ a si mesmo,

b) $H_{Q Q}$ é o acoplamento do subespaço ortogonal a $P$ a si mesmo,

c) $H_{P Q}$ e $H_{Q P}$ são os termos de interação entre $P$ e as coisas ortogonais a $P$, que

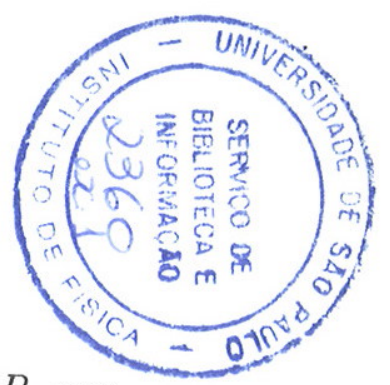
estão $\operatorname{em~} Q$.

\subsubsection{Hamiltoniana efetiva para $P \Phi_{f}$}

Substituindo $Q \Phi_{f}$ na primeira das equações (3.8) obtemos uma Hamiltoniana efetiva para a componente $P \Phi_{f}$,

$$
\left(E-H_{P P}-H_{P Q} \frac{1}{E-H_{Q Q}} H_{Q P}\right) P \Phi_{f}=0 .
$$

Esta hamiltoniana efetiva é, em geral, rapidamente dependente da energia (pólos de $\left(E-H_{Q Q}\right)^{-1}$, "estados de núcleo composto"). É conveniente extrair uma parte média deixando um termo de flutuação, de média nula. Isso é feito através da representação óptica de fundo.

\subsubsection{Representação óptica de fundo}

É neste ponto que introduzimos a "representação óptica de fundo" de Kawai, Kerman e McVoy[21], para nos ajudar a tratar os processos envolvendo os estados de núcleo composto, isto é, separar as flutuações que aparecem na seção de choque daquilo que permanece quando se considera sua média em energia. A importância 
desse formalismo, como já foi dito, reside no fato de podermos obter uma expressão para a seção de choque como a soma de duas contribuições incoerentes:

$$
\left\langle\sigma_{f i}\right\rangle_{I} \propto\left\langle\left|T_{f i}\right|^{2}\right\rangle_{I}=\left|T_{f i}^{l i s a}\right|^{2}+\left\langle\left|T_{f i}^{f l u t .}\right|^{2}\right\rangle_{I}
$$

Vamos obter então, para $P \Phi_{f}$, uma parte "lisa", ou seja, uma função de onda "média" e um termo de flutuação. Esta separação nos deve garantir ainda que ao fazer a média de $T_{f i}$ (para obter a seção de choque média) a parte flutuante vai a zero enquanto a parte "média" fica inalterada.

Para obtermos a separação acima, vamos usar a identidade entre operadores[21]:

$$
H_{P Q} \frac{1}{E-H_{Q Q}+\imath \epsilon} H_{Q P} \equiv H_{P Q} \frac{1}{E-H_{Q Q}+\imath I} H_{Q P}+V_{P Q}^{(+)} \frac{1}{E-H_{Q Q}+\imath \epsilon} V_{Q P}^{(+)}
$$

com

$$
\begin{aligned}
& V_{P Q}^{( \pm)}(E)=H_{P Q} \sqrt{\frac{ \pm \imath I}{E-H_{Q Q} \pm \imath I}} \\
& V_{Q P}^{( \pm)}(E)=\sqrt{\frac{ \pm \imath I}{E-H_{Q Q} \pm \imath I}} H_{Q P} .
\end{aligned}
$$

Por causa do $\imath I$ no denominador, estas funções dependem apenas fracamente da energia (são "lisas").

O primeiro termo do lado direito da equação (3.11) corresponde à média em energia (tomada como uma função Lorentziana de largura $I$ do operador do lado esquerdo), enquanto o segundo é um termo de flutuação rapidamente dependente da energia. Na medida em que o termo que representa a média não se altera quando dele é tomada uma segunda média, a média em energia do termo de flutuação é zero. A importância dos fatores $V_{P Q}$ e $V_{Q P}$, reside nos termos $\left(E-H_{Q Q}+\imath I\right)^{1 / 2}$ pois 
eles limitam as contribuições de flutuação a estados de núcleo composto próximos à energia de interesse $E$. Outra vantagem que esses fatores asseguram é que as fases dos termos se distribuam entre 0 e $2 \pi$, de tal forma que suas contribuições se cancelem em média[21].

Substituindo (3.11) em (3.9) obtemos uma equação complexa efetiva para $P \Phi_{f}$ da forma,

$$
\left(E-\mathcal{H}_{P P}-V_{P Q} \mathcal{G}_{Q}(E) V_{Q P}\right) P \Phi_{f}=0
$$

onde

$$
\mathcal{H}_{P P}=H_{P P}+H_{P Q}\left(E+\imath I-H_{Q Q}\right)^{-1} H_{Q P}
$$

pode ser relacionado a uma hamiltoniana óptica de canais acoplados no espaço de partícula - buraco e

$$
\mathcal{G}_{Q}(E)=\left(E-H_{Q Q}+\imath \epsilon\right)^{-1}
$$

Com condições de contorno apropriadas $P \Phi_{f}$ pode ser dada por

$$
P \Phi_{f}=\chi_{f}+\mathcal{G}_{P P}^{o p t} V_{P Q} \mathcal{G}_{Q}(E) V_{Q P} P \Phi_{f}
$$

cuja solução formal é (restaurando o sinal apropriado),

$$
P\left|\Phi_{f}^{(+)}\right\rangle=\left|\chi_{f}^{(+)}\right\rangle+\mathcal{G}_{P P}^{o p t(+)} V_{P Q}^{(+)} \frac{1}{E^{(+)}-H_{Q Q}-V_{Q P}^{(+)} \mathcal{G}_{P P}^{o p t(+)} V_{P Q}^{(+)}} V_{Q P}^{(+)}\left|\chi_{f}^{(+)}\right\rangle
$$

onde $\mathcal{G}_{P P}^{\text {opt }(+)}(E)=\left(E-\mathcal{H}_{P P}\right)^{-1}$ é a função de Green óptica e $\chi_{f}^{(+)}$é a função de onda óptica de canais acoplados.

Substituindo $P\left|\Phi_{f}^{(+)}\right\rangle$na equação para $T_{f i}$, 


$$
T_{f i} \simeq\left\langle\tilde{\chi}_{f}^{(-)}\left|\mathcal{O}_{e e^{\prime}}\right| \Phi_{i}\right\rangle+\left\langle\tilde{\chi}_{f}^{(-)}\left|V_{P Q}^{(-)} \frac{1}{E^{(-)}-H_{Q Q}-V_{Q P}^{(-)} \mathcal{G}_{P P}^{o p t(-)} V_{P Q}^{(-)}} V_{Q P}^{(-)} \mathcal{G}_{P P}^{o p t(-)} \mathcal{O}_{e e^{\prime}}\right| \Phi_{i}\right\rangle
$$

obtemos uma parte lisa mais um termo de flutuação. Essa é uma expressão para a amplitude de transição exclusiva. Para obtermos a inclusiva, devemos somar sobre $f$ em $\left|T_{f i}\right|^{2}$.

É possível, então, mostrar que a parte lisa pode ser reduzida na expressão da função resposta obtida no tratamento do capítulo 2 . A função de onda óptica satisfaz

$$
\left(E-H_{P P}-H_{P Q} \frac{1}{E+\imath I-H_{Q Q}} H_{Q P}\right)\left|\chi_{f}^{(-)}\right\rangle=0
$$

e a contribuição lisa da $\left|T_{f i}\right|^{2}$ é dada por

$$
\left|T_{f i}^{l i s a}\right|^{2}=\left\langle\Phi_{i}\left|\mathcal{O}_{e e^{\prime}}^{\dagger}\right| \chi_{f}^{(+)}\right\rangle\left\langle\tilde{\chi}_{f}^{(-)}\left|\mathcal{O}_{e e^{\prime}}\right| \Phi_{i}\right\rangle
$$

Usando as expressões acima e somando sobre os estados finais, obtemos a amplitude de espalhamento inclusiva

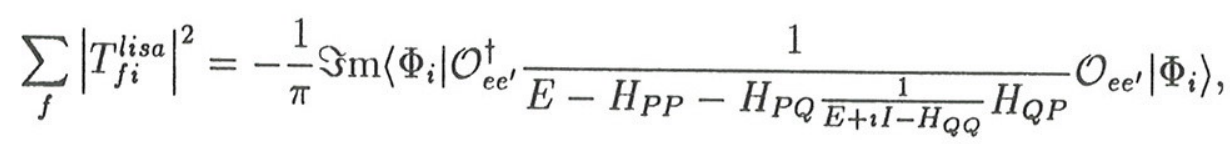

que pode ainda ser escrita numa forma familiar

$$
\begin{aligned}
\sum_{f}\left|T_{f i}^{l i s a}\right|^{2}= & -\frac{1}{\pi} \Im \mathrm{m} \sum_{\substack{p_{j} h_{j} \\
p_{i} h_{i}}}\left\langle\Phi_{i}\left|\mathcal{O}_{e e^{\prime}}^{\dagger}\right| p_{j} h_{j}\right\rangle \\
& \times\left\langle p_{j} h_{j}\left|\frac{1}{E-H_{P P}-H_{P Q} \frac{1}{E+\imath I-H_{Q Q}} H_{Q P}}\right| p_{i} h_{i}\right\rangle\left\langle p_{i} h_{i}\left|\mathcal{O}_{e e^{\prime}}\right| \Phi_{i}\right\rangle .
\end{aligned}
$$

A expressão acima tem a mesma estrutura da equação obtida para a função resposta duas partículas-dois buracos do tratamento do capítulo 2. O estado $\left|\Phi_{i}\right\rangle$ 
corresponde a $|0\rangle$, no capítulo 2, que foi aproximado por um Hartree-Fock $(|H F\rangle)$ e $E$ corresponde à $\omega$. Aqui $p_{i}\left(p_{j}\right)$ e $h_{i}\left(h_{j}\right)$ mantém a mesma definição do capítulo 2 e a rigor, $I$ introduz contribuições imaginárias para o potencial óptico (interação efetiva no espaço PP) que podem ser tomadas como correspondendo, qualitativamente, às médias feitas na seção 3.1.2 .

O primeiro termo do lado direito da equação (3.18) representa a produção de estado partícula - buraco que decai pelo canal óptico para o contínuo. Temos então a seção de choque de espalhamento lisa,

$$
\left\langle\sigma_{f i}^{l i s a}\right\rangle_{I} \propto\left|\left\langle\tilde{\chi}_{f}^{(-)}\left|\mathcal{O}_{e e^{\prime}}\right| \Phi_{i}\right\rangle\right|^{2} .
$$

Introduzindo um conjunto completo de canais abertos $c_{0}$, podemos escrever $T_{f i}^{\text {lisa }}$ como (confira no apêndice D),

$$
T_{f i}^{l i s a}=\sum_{c_{0}} \int d \vec{r}_{0} \chi_{f c_{0}}^{*}\left(\vec{r}_{0}\right) \mathcal{M}_{R c_{0}}\left(\vec{r}_{0}\right)
$$

onde $\chi_{f c_{o}}^{*}=\left\langle\tilde{\chi}_{f}^{(-)} \mid \vec{r}_{0} c_{0}\right\rangle$ e $\mathcal{M}_{R c_{o}}=\left\langle\vec{r}_{0} c_{0}\left|\mathcal{O}_{e e^{\prime}}\right| \Phi_{i}\right\rangle$. Denotamos o canal $A+e$ inicial como $i$ e o canal $C+p$ final como f. $R$ representa a parte do processo da reação direta inicial que conduz aos estados instáveis do núcleo composto $A^{*}$.

\subsubsection{Tratamento das flutuações}

Podemos agora trabalhar com o termo de flutuação da equação (3.18). Este termo liga todos os canais abertos importantes para o decaimento de $A^{*}$ (incluindo a formação de núcleo composto, em $Q$ ), no espaço $P$. Vamos usar uma base biortogonal para os estados $|q\rangle$ com energias complexas $\epsilon_{q}$, que satisfazem

$$
\left(\epsilon_{q}-\mathcal{H}_{Q Q}\right)|q\rangle=0, \quad\left(\epsilon_{q}^{*}-\mathcal{H}_{Q Q}^{\dagger}\right)|\tilde{q}\rangle=0,
$$




$$
\left\langle\tilde{q} \mid q^{\prime}\right\rangle=\delta_{q q^{\prime}}, \quad \sum_{q}|q\rangle\langle\tilde{q}|=Q
$$

onde $\mathcal{H}_{Q Q}=H_{Q Q}-V_{Q P}^{(+)} \mathcal{G}_{P P}^{\text {opt }(+)} V_{P Q}^{(+)}$(veja eq.(3.17)).

Obtemos para $T_{f i}^{f l u t}$ a expressão,

$$
\begin{aligned}
T_{f i}^{f l u t} & =\sum_{q} \frac{\left\langle\tilde{\chi}_{f}^{(-)}\left|V_{P Q}^{(-)}\right| q\right\rangle\left\langle\tilde{q}\left|V_{Q P}^{(-)} \mathcal{G}_{P P}^{\text {opt }(-)} \mathcal{O}_{e e^{\prime}}\right| \Phi_{i}\right\rangle}{E-\epsilon_{q}} \\
& =\sum_{q} \frac{g_{f q} M_{q R}}{E-\epsilon_{q}}
\end{aligned}
$$

sendo

$$
M_{q R} \equiv\left\langle\tilde{q}\left|V_{Q P}^{(-)} \mathcal{G}_{P P}^{\text {opt }(-)} \mathcal{O}_{e e^{\prime}}\right| \Phi_{i}\right\rangle \text { que representa a produção de núcleo composto pelo }
$$
canal de entrada,

$g_{f q} \equiv\left\langle\tilde{\chi}_{f}^{(-)}\left|V_{P Q}^{(-)}\right| q\right\rangle$ é o decaimento do núcleo composto para o contínuo. Os estados $|q\rangle$ estão espaçados à distância $D_{q}$.

Assim como obtemos a equação (3.24), podemos escrever $g_{f q}$ e $M_{q R}$ em termos de canais abertos (confira no apêndice D), ou seja,

$$
g_{f q}=\sum_{c_{1}} \int d \vec{r}_{1} \chi_{f c_{1}}^{*}\left(\vec{r}_{1}\right) V_{q c_{1}}\left(\vec{r}_{1}\right)
$$

e

$$
M_{q R}=\sum_{c_{1}} \int d \vec{r}_{1} V_{q c_{1}}\left(\vec{r}_{1}\right) m_{R c_{1}}^{*}\left(\vec{r}_{1}\right)
$$

onde

$$
V_{q c_{1}}\left(\vec{r}_{1}\right) \equiv\left\langle\vec{r}_{1} c_{1}\left|V_{Q P}^{(-)}\right| q\right\rangle,
$$




$$
\begin{aligned}
\mathcal{G}_{c_{0} c_{1}}^{(E)}\left(\overrightarrow{r_{0}}, \overrightarrow{r_{1}}\right) & \equiv \sum_{c_{2}} \int d E^{\prime} \frac{\left\langle\vec{r}_{1} c_{1} \mid \chi_{c_{2}}^{(+)}\left(E^{\prime}\right)\right\rangle\left\langle\tilde{\chi}_{c_{2}}^{(+)}\left(E^{\prime}\right) \mid \overrightarrow{r_{0}} c_{0}\right\rangle}{E-E^{\prime}-\imath \epsilon}, \\
m_{R c_{1}}^{*}\left(\vec{r}_{1}\right) & =\frac{1}{2 \pi} \sum_{c_{0}} \int d \overrightarrow{r_{0}} \mathcal{G}_{c_{0} c_{1}}^{(E)}\left(\vec{r}_{0}, \vec{r}_{1}\right) \mathcal{M}_{R c_{0}}\left(\vec{r}_{0}\right),
\end{aligned}
$$

na função de Green introduzimos um conjunto completo de funções de onda ópticas no canal $c_{2}$ com energia $E^{\prime}$.

O módulo quadrado da equação (3.26) dá

$$
\left|T_{f i}^{f l u t}\right|^{2}=\sum_{q q^{\prime}} \frac{g_{f q} M_{q R} g_{f q^{\prime}}^{*} M_{q^{\prime} R}^{*}}{\left(E-\epsilon_{q}\right)\left(E-\epsilon_{q^{\prime}}^{*}\right)} .
$$

Tomando a média da seção de choque de flutuação num intervalo médio $I$, conseguimos

$$
\left\langle\sigma_{f i}^{f l u t}\right\rangle_{I} \propto\left\langle\sum_{q q^{\prime}} \frac{g_{f q} M_{q R} g_{f q^{\prime}}^{*} M_{q^{\prime} R}^{*}}{\left(E-\epsilon_{q}\right)\left(E-\epsilon_{q^{\prime}}^{*}\right)}\right\rangle_{I} .
$$

Podemos, a partir de agora, proceder como no trabalho de Kawai, Kerman e McVoy[21] e escrever a seção de choque de flutuação em termos de matrizes $X$. Assim,

$$
\begin{aligned}
\left\langle\sigma_{f i}^{f l u t}\right\rangle_{I} & \propto\left\langle\sum_{q} \frac{\left|g_{f q}\right|^{2}\left|M_{q R}\right|^{2}}{\left|E-\epsilon_{q}\right|^{2}}\right\rangle_{I} \\
& \simeq 2 \pi\left\langle\frac{\left|g_{f q}\right|^{2}\left|M_{q R}\right|^{2}}{D_{q} \Gamma_{q}}\right\rangle_{q} \\
& \simeq \frac{2 \pi}{D \Gamma}\left\langle\left|g_{f q}\right|^{2}\left|M_{q R}\right|^{2}\right\rangle_{q} \\
& \simeq\left[X_{f f} X_{R R}+X_{R f} X_{f R}\right]
\end{aligned}
$$

sendo $D$ e $\Gamma$ os espaçamento e largura médios dos estados.

No trabalho de KKM, os elementos da matriz $X$ são diretamente determinados da matriz $S$-óptica (não unitariedade de $\bar{S}$ ) através da matriz de transmissão 


$$
\mathcal{P}_{c c^{\prime}}=\left(1-\bar{S} \bar{S}^{\dagger}\right)_{c c^{\prime}}
$$

No caso do espalhamento quase-livre elas devem, no entanto, ser substituídas por

$$
\begin{aligned}
X_{R R} & =\left(\frac{2 \pi}{D \Gamma}\right)^{1 / 2}\left\langle M_{q R} M_{q R}^{*}\right\rangle_{q} \\
& =\left(\frac{2 \pi}{D \Gamma}\right)^{1 / 2} \sum_{c_{1}} \iint d \overrightarrow{r_{1}} d \overrightarrow{r_{2}} m_{R c_{1}}^{*}\left(\overrightarrow{r_{1}}\right) m_{R c_{1}}\left(\overrightarrow{r_{2}}\right)\left\langle V_{q c_{1}}\left(\overrightarrow{r_{1}}\right) V_{q c_{1}}^{*}\left(\overrightarrow{r_{2}}\right)\right\rangle_{q},(3, \vec{c}) \\
X_{R f} & =\left(\frac{2 \pi}{D \Gamma}\right)^{1 / 2}\left\langle M_{q R} g_{f q}^{*}\right\rangle_{q} \\
& =\left(\frac{2 \pi}{D \Gamma}\right)^{1 / 2} \sum_{c_{1}} \iint d \overrightarrow{r_{1}} d \overrightarrow{r_{2}} m_{R c_{1}}^{*}\left(\overrightarrow{r_{1}}\right) \chi_{f c_{1}}\left(\overrightarrow{r_{2}}\right)\left\langle V_{q c_{1}}\left(\overrightarrow{r_{1}}\right) V_{q c_{1}}^{*}\left(\overrightarrow{r_{2}}\right)\right\rangle_{q}, \\
X_{f R} & =\left(\frac{2 \pi}{D \Gamma}\right)^{1 / 2}\left\langle g_{f q} M_{q R}^{*}\right\rangle_{q} \\
& =\left(\frac{2 \pi}{D \Gamma}\right)^{1 / 2} \sum_{c_{1}} \iint d \overrightarrow{r_{1}} d \overrightarrow{r_{2}} \chi_{f c_{1}}^{*}\left(\overrightarrow{r_{1}}\right) m_{R c_{1}}\left(\overrightarrow{r_{2}}\right)\left\langle V_{q c_{1}}\left(\overrightarrow{r_{1}}\right) V_{q c_{1}}^{*}\left(\overrightarrow{r_{2}}\right)\right\rangle_{q}
\end{aligned}
$$

e

$$
\begin{aligned}
X_{f f} & =\left(\frac{2 \pi}{D \Gamma}\right)^{1 / 2}\left\langle g_{f q} g_{f q}^{*}\right\rangle_{q} \\
& =\left(\frac{2 \pi}{D \Gamma}\right)^{1 / 2} \sum_{c_{1}} \iint d \overrightarrow{r_{1}} d \overrightarrow{r_{2}} \chi_{f c_{1}}^{*}\left(\overrightarrow{r_{1}}\right) \chi_{f c_{1}}\left(\overrightarrow{r_{2}}\right)\left\langle V_{q c_{1}}\left(\overrightarrow{r_{1}}\right) V_{q c_{1}}^{*}\left(\overrightarrow{r_{2}}\right)\right\rangle_{q}
\end{aligned}
$$

Para calcular as matrizes $X$-extendidas usamos o método desenvolvido por Kerman e McVoy no qual, em vez de determinar $X$ de uma equação de unitariedade, usa-se diretamente a equação (3.34) e calcula-se o termo $\left\langle V_{q c_{1}}\left(\overrightarrow{r_{1}}\right) V_{q c_{2}}^{*}\left(\overrightarrow{r_{2}}\right)\right\rangle_{q}$ por uma aproxi- 
mação que o relaciona com a parte imaginária do potencial óptico, $W_{c_{1}}\left(\overrightarrow{r_{1}}\right)$, no canal $c_{1}$. Uma vez que o cálculo está desenvolvido em detalhes na referência[23], usaremos diretamente o resultado

$$
\left\langle V_{q c_{1}}\left(\overrightarrow{r_{1}}\right) V_{q c_{2}}^{*}\left(\overrightarrow{r_{2}}\right)\right\rangle_{q} \approx \delta_{c_{1} c_{2}} \delta\left(\overrightarrow{r_{1}}-\overrightarrow{r_{2}}\right) \sqrt{\frac{D \Gamma}{2 \pi}} \frac{4 \pi}{(\operatorname{Tr} \mathcal{P})^{1 / 2}} W_{c_{1}}\left(\overrightarrow{r_{1}}\right) .
$$

Substituindo a expressão acima nas equações (3.34) à (3.37) obtemos os elementos da matriz $X$-extendida necessários para a seção de choque de flutuação,

$$
\begin{aligned}
& X_{R R} \operatorname{Tr} X \simeq 4 \pi \sum_{c_{1}} \int d \overrightarrow{r_{1}} m_{R c_{1}}^{*}\left(\overrightarrow{r_{1}}\right) W_{c_{1}}\left(\overrightarrow{r_{1}}\right) m_{R c_{1}}\left(\overrightarrow{r_{1}}\right) \\
& X_{R f} \operatorname{Tr} X \simeq 4 \pi \sum_{c_{1}} \int d \overrightarrow{r_{1}} m_{R c_{1}}^{*}\left(\overrightarrow{r_{1}}\right) W_{c_{1}}\left(\overrightarrow{r_{1}}\right) \chi_{f c_{1}}\left(\overrightarrow{r_{1}}\right) \\
& X_{f R} \operatorname{Tr} X \simeq 4 \pi \sum_{c_{1}} \int d \overrightarrow{r_{1}} \chi_{f c_{1}}^{*}\left(\overrightarrow{r_{1}}\right) W_{c_{1}}\left(\overrightarrow{r_{1}}\right) m_{R c_{1}}\left(\overrightarrow{r_{1}}\right)
\end{aligned}
$$

e

$$
X_{f f} \operatorname{Tr} X \simeq 4 \pi \sum_{c_{1}} \int d \overrightarrow{r_{1}} \chi_{f c_{1}}^{*}\left(\overrightarrow{r_{1}}\right) W_{c_{1}}\left(\overrightarrow{r_{1}}\right) \chi_{f c_{1}}\left(\overrightarrow{r_{1}}\right)
$$

Estes resultados junto com a equação (3.32) determinam a seção de choque de flutuação sequencial inteiramente em termos de quantidades obtidas de uma análise óptica de canais acoplados das reações de dois corpos.

No limite em que o número de canais abertos diretamente acoplados é grande, a matriz $X$ é dada diretamente em termos da matriz de transmissão (equação (3.33)) pela expressão

$$
X_{f i} \simeq \frac{\mathcal{P}_{f i}}{[\operatorname{Tr} \mathcal{P}]^{1 / 2}}
$$

e a seção de choque de flutuação é reduzida na fórmula de Hauser-Feshbach: 


$$
\sigma_{f i}^{\text {flut }} \propto \frac{\mathcal{P}_{f} \mathcal{P}_{i}}{\sum_{f^{\prime}} \mathcal{P}_{f^{\prime}}} .
$$




\section{Capítulo 4}

\section{Conclusões}

Neste trabalho apresentamos dois formalismos distintos que podem ser usados para investigar a seção de choque de espalhamento.

No capítulo 2 desenvolvemos o formalismo da função resposta linear que é obtida através da técnica das equações de movimento de operadores. Usamos esse tratamento para obter expressões para a resposta longitudinal.

Uma aplicação numérica deste método é feita para espalhamento de elétrons na região quase-elástica, no modelo do gás de Fermi, para um momento transferido $q=550 \mathrm{MeV} / \mathrm{c}$. Os aspectos novos desse tratamento, envolvem a escolha de técnicas de discretização que possibilitam obter a resposta através da inversão sucessiva de matrizes numéricas.

Investigamos a resposta longitudinal partícula-buraco e duas partículas-dois buracos quando forças de alcance zero e alcance finito são adotadas como as interações residuais.

Algumas conclusões importantes são tiradas destes cálculos. A resposta longitudinal, já ao nível de partícula-buraco, é muito sensível ao tipo de interação p-h usada. Entretanto, é possível descrever os efeitos da interação de alcance finito através de uma interação de alcance zero do tipo Landau-Migdal. 
Com a interação de Landau-Migdal de alcance zero, houve uma redução significativa na intensidade da resposta longitudinal p-h e o pico foi deslocado para $\nu$ maiores, em relação à resposta livre. Com uma interação de alcance finito, mais realística, não houve uma mudança significativa, tanto em termos qualitativos quanto quantitativos.

O propósito central deste capítulo era investigar a contribuição das excitações duas partículas-dois buracos.

A inclusão dos estados de duas partículas-dois buracos no cálculo numérico da resposta longitudinal mostrou resultados muito interessantes. $\mathrm{O}$ efeito das excitações $2 \mathrm{p}-2 \mathrm{~h}$ foi espalhar a resposta longitudinal em $\omega$ e deslocar o pico para energias menores. Mas o resultado mais atraente, está na região de $\omega$ acima de $350 \mathrm{MeV}$ onde obtemos uma intensidade aproximadamente uniforme da resposta (nesta região não há medidas experimentais confiáveis). Isto pode dar conta do "quenching" da regra de soma. A regra de soma experimental, no caso do ${ }^{56} \mathrm{Fe}$ investigado neste trabalho com momento transferido $q=550 \mathrm{MeV} / \mathrm{c}$, dá uma carga atômica menor do que a esperada.

No capítulo 3 apresentamos o formalismo que permite separar a seção de choque de espalhamento em contribuições lisa e de flutuação. Temos a vantagem de podermos usar fenomenologia para obtermos uma solução da seção de choque, incluindo um termo de flutuação inacessível aos tratamentos do tipo usado no capítulo 2. Mostramos também, a partir da formulação em termos de teoria de reações, que a amplitude de espalhamento lisa pode ser reduzida na função resposta do capítulo 2. 


\section{Apêndice A}

\section{Identidades}

As expressões para a função resposta dadas pelas equações (2.26) e (2.28) são facilmente transformadas uma na outra, com a ajuda das identidades de operador[25]:

$$
\begin{gathered}
P \frac{1}{W} P \equiv P \frac{1}{W_{P P}-W_{P Q} \frac{1}{W_{Q Q}} W_{Q P}} P \\
P \frac{1}{W} Q \\
\equiv P \frac{1}{W_{P P}-W_{P Q} \frac{1}{W_{Q Q}} W_{Q P}} W_{P Q} \frac{1}{W_{Q Q}} Q \\
\equiv P \frac{1}{W_{P P}} W_{P Q} \frac{1}{W_{Q Q}-W_{Q P} \frac{1}{W_{P P}} W_{P Q}} Q
\end{gathered}
$$

e

$\frac{1}{W_{P P}-W_{P Q} \frac{1}{W_{Q Q}} W_{Q P}} \equiv \frac{1}{W_{P P}}+\frac{1}{W_{P P}} W_{P Q} \frac{1}{W_{Q Q}-W_{Q P} \frac{1}{W_{P P}} W_{P Q}} W_{Q P} \frac{1}{W_{P P}}$. 


\section{Apêndice B}

\section{Matrizes $P H P, P H Q$ e $Q H Q$}

$$
\begin{aligned}
P H P \equiv & \left\langle p_{j} h_{j}|H| p_{i} h_{i}\right\rangle \\
= & \left(\epsilon_{p_{i}}-\epsilon_{h_{j}}\right) \delta_{p_{j} p_{i}} \delta_{h_{i} h_{j}}+\left\langle h_{i} p_{j}|\tilde{V}| p_{i} h_{j}\right\rangle \\
Q H P \equiv & \left\langle p_{j_{1}} h_{j_{1}} p_{j_{2}} h_{j_{2}}|H| p_{i} h_{i}\right\rangle \\
= & 2\left\langle p_{j_{1}} p_{j_{2}}|\tilde{V}| h_{j_{1}} p_{i}\right\rangle \delta_{h_{i} h_{j_{2}}}+2\left\langle p_{j_{1}} h_{i}|\tilde{V}| h_{j_{2}} h_{j_{1}}\right\rangle \delta_{p_{j_{2}} p_{i}} \\
Q H Q \equiv & \left\langle p_{i_{1}} h_{i_{1}} p_{i_{2}} h_{i_{2}}|H| p_{j_{1}} h_{j_{1}} p_{j_{2}} h_{j_{2}}\right\rangle \\
= & \left(\epsilon_{p_{i_{1}}}+\epsilon_{p_{i_{2}}}-\epsilon_{h_{j_{1}}}-\epsilon_{h_{j_{2}}}\right) \delta_{p_{i_{1}} p_{j_{1}}} \delta_{p_{i_{2}} p_{j_{2}}} \delta_{h_{j_{1}} h_{i_{1}}} \delta_{h_{j_{2}} h_{i_{2}}} \\
& +\left\langle p_{i_{1}} p_{i_{2}}|\tilde{V}| p_{j_{1}} p_{j_{2}}\right\rangle \delta_{h_{j_{1}} h_{i_{1}}} \delta_{h_{j_{2}} h_{i_{2}}} \\
& +\left\langle h_{j_{1}} h_{j_{2}}|\tilde{V}| h_{i_{1}} h_{i_{2}}\right\rangle \delta_{p_{i_{1}} p_{j_{1}}} \delta_{{p_{i}}_{2} p_{j_{2}}} \\
& +2\left\langle p_{i_{1}} h_{j_{2}}|\tilde{V}| h_{i_{2}} p_{j_{1}}\right\rangle \delta_{p_{i_{2}} p_{j_{2}}} \delta_{h_{j_{1}} h_{i_{1}}} \\
& +2\left\langle p_{i_{1}} h_{j_{1}}|\tilde{V}| h_{i_{1}} p_{j_{1}}\right\rangle \delta_{p_{i_{2}} p_{j_{2}}} \delta_{h_{j_{2}} h_{i_{2}}},
\end{aligned}
$$

onde $\left\langle h_{i} p_{j}|\tilde{V}| p_{i} h_{j}\right\rangle=\left\langle h_{i} p_{j}|V| p_{i} h_{j}-h_{j} p_{i}\right\rangle$. 
Vamos escrever as equações acima introduzindo a notação completa de spin e isospin:

$$
\begin{aligned}
& P H P=\left\langle\left[p_{j} h_{j}\right] S M_{S} T M_{T}|H|\left[p_{i} h_{i}\right] S M_{S} T M_{T}\right\rangle
\end{aligned}
$$

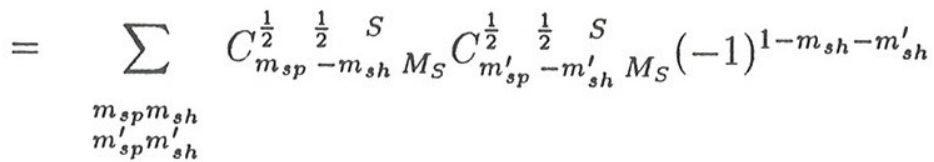

$$
\begin{aligned}
& \times \sum_{\substack{m_{t p} m_{t h} \\
m_{t p}^{\prime} m_{t h}^{\prime}}} C_{m_{t p}-m_{t h} M_{T}}^{\frac{1}{2}} C_{m_{t p}^{\prime}-m_{t h}^{\prime} M_{T}^{\prime}}^{\frac{1}{2}} \underset{1}{\frac{1}{2}} \stackrel{T}{T}(-1)^{1-m_{t h}-m_{t h}} \\
& \times\left\langle H F\left|a_{p_{j}} a_{h_{j}}^{\dagger} H a_{p_{i}}^{\dagger} a_{h_{i}}\right| H F\right\rangle
\end{aligned}
$$

onde

$$
\begin{aligned}
& \left\langle H F\left|a_{p_{j}} a_{h_{j}}^{\dagger} H a_{p_{i}}^{\dagger} a_{h_{i}}\right| H F\right\rangle=\frac{\hbar^{2}}{2 m}\left(k_{p}^{2}-k_{h}^{2}\right) \delta_{\vec{k}_{p} \vec{k}_{p}^{\prime}} \delta_{\vec{k}_{h} \vec{k}_{h}^{\prime}} \\
& \times \delta_{m_{s p} m_{s p}^{\prime}} \delta_{m_{t p} m_{t p}^{\prime}} \delta_{m_{s h} m_{s h}^{\prime}} \delta_{m_{t h} m_{t h}^{\prime}}
\end{aligned}
$$

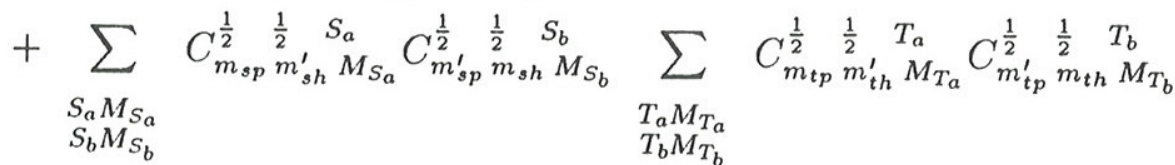

$$
\begin{aligned}
& \times\left\langle\vec{k}_{h} \vec{k}_{p}^{\prime} S_{b} M_{S_{b}} T_{b} M_{T_{b}}|\tilde{V}| \vec{k}_{p} \vec{k}_{h}^{\prime} S_{a} M_{S_{a}} T_{a} M_{T_{a}}\right\rangle .
\end{aligned}
$$

O primeiro passo é calcular o último bracket com a interação escolhida e em seguida tentar reduzir os coeficientes de Clebsch-Gordan na função de Racah[33]. Feito isso obtemos as expressões do capítulo 2. Seguimos o mesmo procedimento para as demais equações.

$$
\begin{aligned}
& Q H P=\left\langle\left[\left[p_{j_{1}}\right] h_{j_{1}} S_{1}^{\prime} T_{1}^{\prime} p_{j_{2}} h_{j_{2}}\right] S_{2}^{\prime} T_{2}^{\prime} ; S_{12}^{\prime} M_{S_{12}}^{\prime} T_{12}^{\prime} M_{T_{12}}^{\prime}|H|\left[p_{i} h_{i}\right] S M_{S} T M_{T}\right\rangle
\end{aligned}
$$

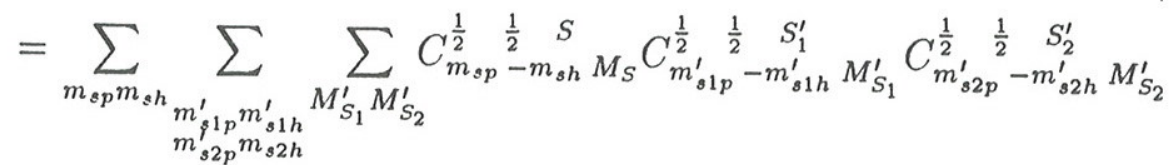




$$
\begin{aligned}
& \times C_{M_{S_{1}}^{\prime}}^{S_{1}^{\prime}} M_{S_{2}}^{\prime} M_{S_{12}}^{\prime} S_{12}^{\prime}(-1)^{\frac{3}{2}-m_{s h}-m_{s 1 h}^{\prime}-m_{s 2 h}^{\prime}}
\end{aligned}
$$

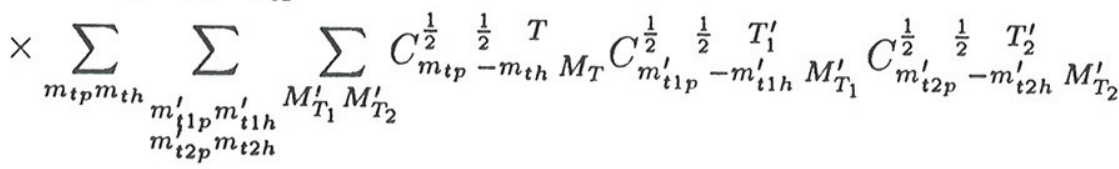

$$
\begin{aligned}
& \times C_{M_{T_{1}}^{\prime}}^{T_{1}^{\prime}} M_{T_{2}}^{T_{2}^{\prime}} M_{T_{12}}^{\prime} T_{12}^{\prime}(-1)^{\frac{3}{2}-m_{t h}-m_{t 1 h}^{\prime}-m_{t 2 h}^{\prime}} \\
& \times\left\langle H F\left|a_{p_{j_{1}}} a_{h_{j_{1}}}^{\dagger} a_{p_{j_{2}}} a_{h_{j_{2}}}^{\dagger} H a_{p_{i}}^{\dagger} a_{h_{i}}\right| H F\right\rangle
\end{aligned}
$$

onde

$$
\begin{aligned}
& \left\langle H F\left|a_{p_{\jmath_{1}}} a_{h_{j_{1}}}^{\dagger} a_{p_{\jmath_{2}}} a_{h_{\jmath_{2}}}^{\dagger} H a_{p_{i}}^{\dagger} a_{h_{i}}\right| H F\right\rangle=
\end{aligned}
$$

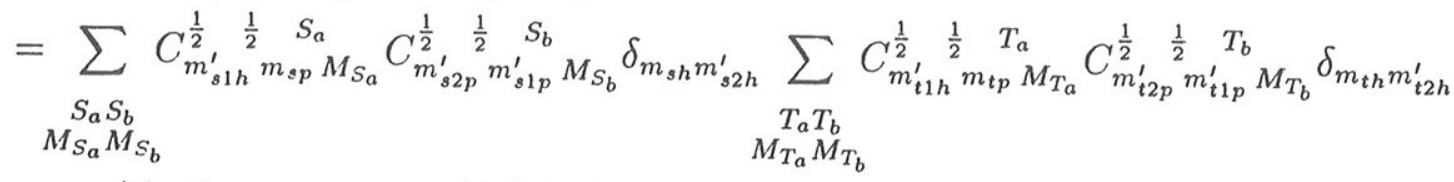

$$
\begin{aligned}
& \times 2\left\langle\vec{k}_{1 p}^{\prime} \vec{k}_{2 p}^{\prime} S_{b} M_{S_{b}} T_{b} M_{T_{b}}|\tilde{V}| \vec{k}_{1 h}^{\prime} \vec{k}_{p} S_{a} M_{S_{a}} T_{a} M_{T_{a}}\right\rangle \delta_{\vec{k}_{h} \vec{k}_{2 h}^{\prime}}
\end{aligned}
$$

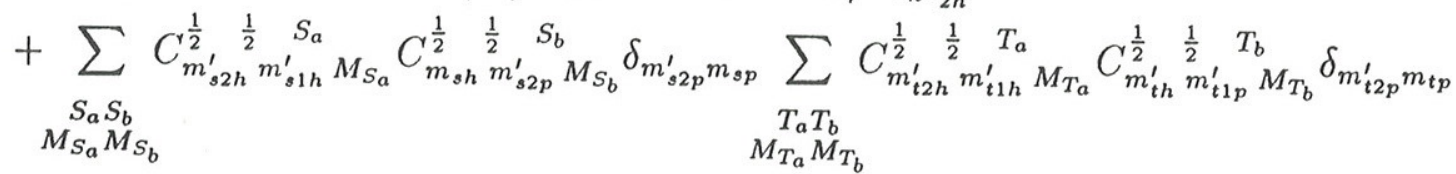

$$
\begin{aligned}
& \times 2\left\langle\vec{k}_{1 p}^{\prime} \vec{k}_{h} S_{b} M_{S_{b}} T_{b} M_{T_{b}}|\tilde{V}| \vec{k}_{2 h}^{\prime} \vec{k}_{1 h}^{\prime} S_{a} M_{S_{a}} T_{a} M_{T_{a}}\right\rangle \delta_{\vec{k}_{2 p}^{\prime} \vec{k}_{p}} .
\end{aligned}
$$

A última equação é dada a seguir.

$$
\begin{aligned}
& Q H Q=\left\langle\left[p_{i_{1}} h_{i_{1}}\right] S_{1} T_{1}\left[p_{i_{2}} h_{i_{2}}\right] S_{2} T_{2} ; S_{12} M_{S_{12}} T_{12} M_{T_{12}}\right| H \\
& \left|\left[p_{j_{1}} h_{j_{1}}\right] S_{1}^{\prime} T_{1}^{\prime}\left[p_{j_{2}} h_{j_{2}}\right] S_{2}^{\prime} T_{2}^{\prime} ; S_{12}^{\prime} M_{S_{12}}^{\prime} T_{12}^{\prime} M_{T_{12}}^{\prime}\right\rangle
\end{aligned}
$$

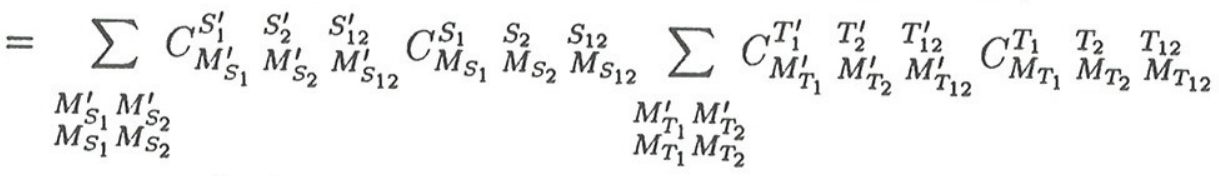

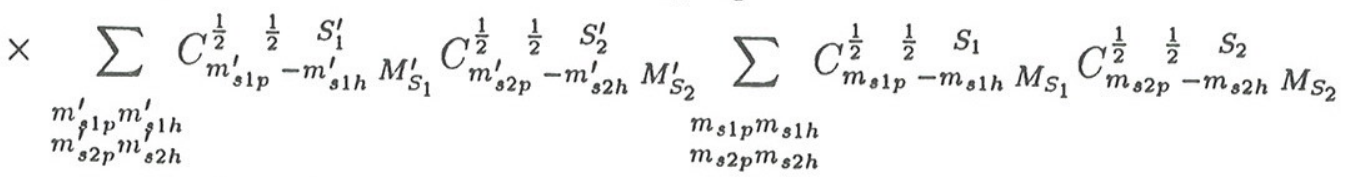

$$
\begin{aligned}
& \times(-1)^{2-m_{s 1 h}^{\prime}-m_{s 2 h}^{\prime}-m_{s 1 h}-m_{s 2 h}}
\end{aligned}
$$




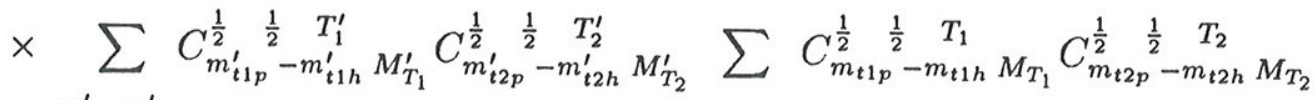

$$
\begin{aligned}
& \begin{array}{ll}
m_{\{1 p}^{\prime} m_{t 1 h}^{\prime} & m_{t 1 p} m_{t 1 h} \\
m_{t 2 p}^{\prime} m_{t 2 h}^{\prime} & m_{t 2 p} m_{t 2 h}
\end{array} \\
& \times(-1)^{2-m_{t 1 h}^{\prime}-m_{t 2 h}^{\prime}-m_{t 1 h}-m_{t 2 h}} \\
& \times\left\langle H F\left|a_{p_{i_{1}}} a_{h_{i_{1}}}^{\dagger} a_{p_{i_{2}}} a_{h_{i_{2}}}^{\dagger} H a_{p_{j_{1}}}^{\dagger} a_{h_{j_{1}}} a_{p_{j_{2}}}^{\dagger} a_{h_{j_{2}}}\right| H F\right\rangle
\end{aligned}
$$

onde

$$
\begin{aligned}
& \left\langle H F\left|a_{p_{i_{1}}} a_{h_{i_{1}}}^{\dagger} a_{p_{i_{2}}} a_{h_{i_{2}}}^{\dagger} H a_{p_{j_{1}}}^{\dagger} a_{h_{j_{1}}} a_{p_{j_{2}}}^{\dagger} a_{h_{j_{2}}}\right| H F\right\rangle= \\
& =\frac{\hbar^{2}}{2 m}\left(k_{1 p}^{2}+k_{2 p}^{2}-k_{1 h}^{2}-h_{2 h}^{2}\right) \delta_{\vec{k}_{1 p} \vec{k}_{1 p}^{\prime}} \delta_{\vec{k}_{2 p} \vec{k}_{2 p}^{\prime}} \delta_{\vec{k}_{1 h}^{\prime} \vec{k}_{1 h}} \delta_{\vec{k}_{2 h}^{\prime} \vec{k}_{2 h}} \\
& \times \delta_{m_{s 1 p} m_{s 1 p}^{\prime}} \delta_{m_{s 2 p} m_{s 2 p}^{\prime}} \delta_{m_{s 1 h}^{\prime} m_{s 1 h}} \delta_{m_{s 2 h}^{\prime} m_{s 2 h}} \delta_{m_{t 1 p} m_{t 1 p}^{\prime}} \delta_{m_{t 2 p} m_{t 2 p}^{\prime}} \delta_{m_{t 1 h}^{\prime} m_{t 1 h}} \delta_{m_{t 2 h}^{\prime} m_{t 2 h}}
\end{aligned}
$$

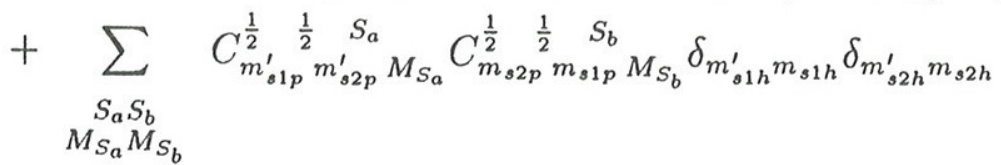

$$
\begin{aligned}
& \times \sum_{\substack{T_{a} T_{b} \\
M_{T_{a}} M_{T_{b}}}} C_{m_{t 1 p}^{\prime} m_{t 2 p}^{\prime} M_{T_{a}}}^{\frac{1}{2}} C_{m_{t 2 p} m_{t 1 p} M_{T_{b}}}^{\frac{1}{2}} \delta_{m_{t 1 h}^{\prime} m_{t 1 h}}^{\frac{1}{2} T_{b}} \delta_{m_{t 2 h}^{\prime} m_{t 2 h}} \\
& \times\left\langle\vec{k}_{1 p} \vec{k}_{2 p} S_{b} M_{S_{b}} T_{b} M_{T_{b}}|\tilde{V}| \vec{k}_{1 p}^{\prime} \vec{k}_{2 p}^{\prime} S_{a} M_{S_{a}} T_{a} M_{T_{a}}\right\rangle \delta_{\vec{k}_{1 h}^{\prime} \vec{k}_{1 h}} \delta_{\vec{k}_{2 h}^{\prime} \vec{k}_{2 h}}
\end{aligned}
$$

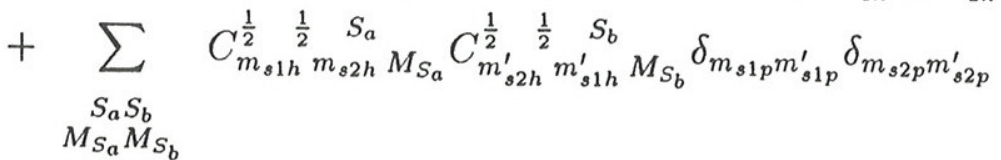

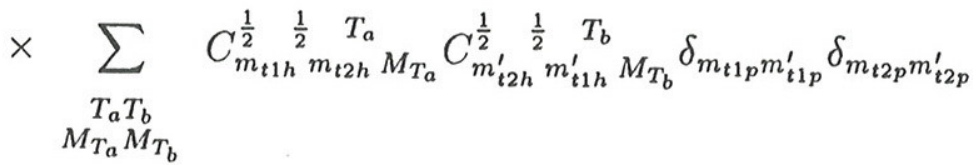

$$
\begin{aligned}
& \times\left\langle\vec{k}_{1 h}^{\prime} \vec{k}_{2 h}^{\prime} S_{b} M_{S_{b}} T_{b} M_{T_{b}}|\tilde{V}| \vec{k}_{1 h} \vec{k}_{2 h} S_{a} M_{S_{a}} T_{a} M_{T_{a}}\right\rangle \delta_{\vec{k}_{1 p} \vec{k}_{1 p}^{\prime}} \delta_{\vec{k}_{2 p} \vec{k}_{2 p}^{\prime}}
\end{aligned}
$$

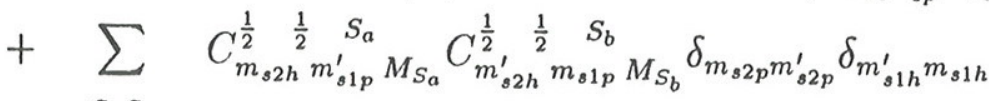

$$
\begin{aligned}
& \begin{array}{c}
S_{a} S_{b} \\
M_{S_{a}} M_{S_{b}}
\end{array}
\end{aligned}
$$

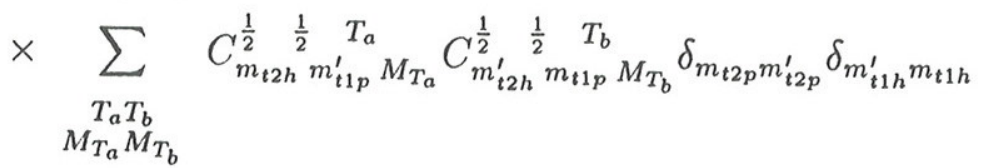

$$
\begin{aligned}
& \times 2\left\langle\vec{k}_{1 p} \vec{k}_{2 h}^{\prime} S_{b} M_{S_{b}} T_{b} M_{T_{b}}|\tilde{V}| \vec{k}_{2 h} \vec{k}_{1 p}^{\prime} S_{a} M_{S_{a}} T_{a} M_{T_{a}}\right\rangle \delta_{\vec{k}_{2 p} \vec{k}_{2 p}^{\prime}} \delta_{\vec{k}_{1 h}^{\prime} \vec{k}_{1 h}}
\end{aligned}
$$




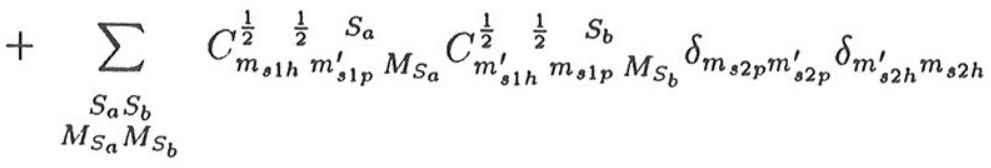

$$
\begin{aligned}
& \times \sum_{\substack{T_{a} T_{b} \\
M_{T_{a}} M_{T_{b}}}} C_{m_{t 1 h}{ }^{\frac{1}{2}} m_{t 1 p}^{\prime} M_{T_{a}}}^{\frac{1}{T_{a}}} C_{m_{t 1 h}^{\prime} m_{t 1 p}}^{\frac{1}{2}} \stackrel{\frac{1}{2} M_{T_{b}}}{T_{b}} \delta_{m_{t 2 p} m_{t 2 p}^{\prime}} \delta_{m_{t 2 h}^{\prime} m_{t 2 h}} \\
& \times 2\left\langle\vec{k}_{1 p} \vec{k}_{1 h}^{\prime} S_{b} M_{S_{b}} T_{b} M_{T_{b}}|\tilde{V}| \vec{k}_{1 h} \vec{k}_{1 p}^{\prime} S_{a} M_{S_{a}} T_{a} M_{T_{a}}\right\rangle \delta_{\vec{k}_{2 p} \vec{k}_{2 p}^{\prime}} \delta_{\vec{k}_{2 h}^{\prime} \vec{k}_{2 h}}
\end{aligned}
$$

67 


\section{Apêndice C}

\section{Expressões para determinar o número total de estados $2 \mathrm{p}-2 \mathrm{~h}$}

Neste apêndice vamos colocar duas expressões úteis que podem ser usadas para determinar o número total de estados para os decaimentos do buraco e da partícula. Os histogramas do capítulo 2 foram obtidos através do cálculo das expressões abaixo.

A equação abaixo refer-se a autoenergia do buraco (figura (2.7a)): $\vec{k}_{h_{2}}^{\prime}+\vec{k}_{h_{1}}^{\prime}=$ $\vec{k}_{h}+\vec{k}_{p_{1}}^{\prime}$.

$$
\begin{aligned}
I= & \int d \vec{k}_{h_{2}}^{\prime} \int d \vec{k}_{h_{1}}^{\prime} \int d \vec{k}_{p_{1}}^{\prime} \theta\left(k_{F}-k_{h_{2}}^{\prime}\right) \theta\left(k_{F}-k_{h_{1}}^{\prime}\right) \theta\left(k_{p_{1}}^{\prime}-k_{F}\right) \\
& \times \delta\left(\vec{k}_{h_{2}}^{\prime}+\vec{k}_{h_{1}}^{\prime}-\vec{k}_{p_{1}}^{\prime}-\vec{k}_{h}\right) \delta\left(\frac{\hbar^{2}}{2 m}\left(k_{p}^{2}+{k_{p_{1}}^{\prime 2}}^{2}-k_{h_{2}}^{\prime 2}-k_{h_{1}}^{\prime 2}\right)-E_{2}\right)
\end{aligned}
$$

Usando coordenadas esféricas chegamos no seguinte resultado:

$$
\begin{aligned}
I= & \int_{0}^{k_{F}}{k^{\prime}}_{h_{1}}^{2} d k_{h_{1}}^{\prime} \int_{0}^{\pi} \sin \theta_{h_{1}} d \theta_{h_{1}} \int_{0}^{2 \pi} d \varphi_{h_{1}} \int_{0}^{\pi} \sin \theta_{p_{1}} d \theta_{p_{1}} \int_{0}^{2 \pi} d \varphi_{p_{1}} \\
& \times K^{2} \frac{1}{|a|} \theta\left(k_{F}-\sqrt{K_{B}}\right) \theta\left(k_{F}-k_{h_{1}}^{\prime}\right) \theta\left(K-k_{F}\right)
\end{aligned}
$$

onde 


$$
\begin{gathered}
K=\frac{2 k_{F}^{2} \nu-k_{p}^{2}+k_{h}^{2}+2 k_{h_{1}}^{\prime 2}-2 k_{h} k_{h_{1}}^{\prime} \cos \alpha_{h h_{1}}}{2\left(k_{h_{1}}^{\prime} \cos \alpha_{p_{1} h_{1}}-k_{h} \cos \alpha_{p_{1} h}\right)}, \\
\frac{1}{|a|}=\frac{1}{\frac{\hbar^{2}}{m}\left|k_{h_{1}}^{\prime} \cos \alpha_{p_{1} h_{1}}-k_{h} \cos \alpha_{p_{1} h}\right|},
\end{gathered}
$$

sendo

$$
\begin{gathered}
\nu=E_{2} \frac{m}{\hbar^{2} k_{F}^{2}}, \\
K_{B}=K^{2}-2 k_{F}^{2} \nu+k_{p}^{2}-k_{h_{1}}^{2},
\end{gathered}
$$

$$
\begin{aligned}
\cos \alpha_{h h_{1}} & =\cos \theta_{h} \cos \theta_{h_{1}}+\sin \theta_{h} \sin \theta_{h_{1}} \cos \left(\varphi_{h}-\varphi_{h_{1}}\right), \\
\cos \alpha_{p_{1} h_{1}} & =\cos \theta_{p_{1}} \cos \theta_{h_{1}}+\sin \theta_{p_{1}} \sin \theta_{h_{1}} \cos \left(\varphi_{p_{1}}-\varphi_{h_{1}}\right), \\
\cos \alpha_{p_{1} h} & =\cos \theta_{p_{1}} \cos \theta_{h}+\sin \theta_{p_{1}} \sin \theta_{h} \cos \left(\varphi_{p_{1}}-\varphi_{h}\right) .
\end{aligned}
$$

A equação abaixo refer-se a autoenergia da partícula (figura (2.7b)): $\vec{k}_{p}+\vec{k}_{h_{1}}^{\prime}=$ $\vec{k}_{p_{2}}^{\prime}+\vec{k}_{p_{1}}^{\prime}$.

$$
\begin{aligned}
I= & \int d \vec{k}_{p_{2}}^{\prime} \int d \vec{k}_{p_{1}}^{\prime} \int d \vec{k}_{h_{1}}^{\prime} \theta\left(k_{p_{2}}^{\prime}-k_{F}\right) \theta\left(k_{p_{1}}^{\prime}-k_{F}\right) \theta\left(k_{F}-k_{h_{1}}^{\prime}\right) \\
& \times \delta\left(\vec{k}_{p_{2}}^{\prime}-\vec{k}_{h_{1}}^{\prime}-\vec{k}_{p}+\vec{k}_{p_{1}}^{\prime}\right) \delta\left(\frac{\hbar^{2}}{2 m}\left(k_{p_{1}}^{\prime 2}+k_{p_{2}}^{\prime 2}-k_{h_{1}}^{\prime 2}-k_{h}^{2}\right)-E_{2}\right) .
\end{aligned}
$$

Usando coordenadas esféricas chegamos no seguinte resultado:

$$
\begin{aligned}
I & =\int k_{p_{1}}^{\prime 2} d k_{p_{1}}^{\prime} \int \sin \theta_{p_{1}} d \theta_{p_{1}} \int d \varphi_{p_{1}} \int k_{h_{1}}^{\prime 2} d k_{h_{1}}^{\prime} \int \sin \theta_{h_{1}} d \theta_{h_{1}} \int d \varphi_{h_{1}} \\
& \times \frac{m}{\hbar^{2}} \frac{1}{2|a|} \theta\left(k_{F}-k_{h_{1}}^{\prime}\right) \theta(\arg (a)) \\
& \times\left[K_{+a}^{2} \theta\left(\sqrt{K_{P_{(+a)}}}-k_{F}\right) \theta\left(K_{+a}-k_{F}\right)+K_{-a}^{2} \theta\left(\sqrt{K_{P_{(-a)}}}-k_{F}\right) \theta\left(K_{-a}-k_{F}\right)\right]
\end{aligned}
$$


onde

$$
\begin{aligned}
a= & \frac{1}{2} \sqrt{\left(k_{p} \cos \alpha_{p p_{1}}+k_{h_{1}}^{\prime} \cos \alpha_{h_{1} p_{1}}\right)^{2}-2 k_{p}^{2}-2 k_{h}^{2}+4 k_{F}^{2} \nu-4 k_{h_{1}}^{\prime} k_{p} \cos \alpha_{h_{1} p}}, \\
K_{+a}= & \frac{1}{2}\left(k_{p} \cos \alpha_{p p_{1}}+k_{h_{1}}^{\prime} \cos \alpha_{h_{1} p_{1}}\right)+a \\
K_{-a}= & \frac{1}{2}\left(k_{p} \cos \alpha_{p p_{1}}+k_{h_{1}}^{\prime} \cos \alpha_{h_{1} p_{1}}\right)-a \\
K_{P_{(+a)}=} & k_{h_{1}}^{\prime 2}+k_{p}^{2}+K_{+a}^{2}-2 K_{+a}\left(k_{p} \cos \alpha_{p p_{1}}+k_{h_{1}}^{\prime} \cos \alpha_{h 1 p_{1}}\right)+2 k_{h_{1}}^{\prime} k_{p} \cos \alpha_{h_{1} p}, \\
K_{P_{(-a)}=}= & k_{h_{1}}^{\prime 2}+k_{p}^{2}+K_{-a}^{2}-2 K_{-a}\left(k_{p} \cos \alpha_{p p_{1}}+k_{h_{1}}^{\prime} \cos \alpha_{h 1 p_{1}}\right)+2 k_{h_{1}}^{\prime} k_{p} \cos \alpha_{h_{1} p} . \\
& \cos \alpha_{p p_{1}}=\cos \theta_{p} \cos \theta_{p_{1}}+\sin \theta_{p} \sin \theta_{p_{1}} \cos \left(\varphi_{p}-\varphi_{p_{1}}\right) \\
& \cos \alpha_{h_{1} p_{1}}=\cos \theta_{h_{1}} \cos \theta_{p_{1}}+\sin \theta_{h_{1}} \sin \theta_{p_{1}} \cos \left(\varphi_{h_{1}}-\varphi_{p_{1}}\right), \\
& \cos \alpha_{h_{1} p}=\cos \theta_{h_{1}} \cos \theta_{p}+\sin \theta_{h_{1}} \sin \theta_{p} \cos \left(\varphi_{h_{1}}-\varphi_{p}\right) .
\end{aligned}
$$

Condição para $\theta(\arg (a)): \arg (a)$ pode ter valores positivos e negativos. Se negativos não tem solução real.

O número total de estados no espaço de duas partículas-dois buracos é dado por:

$$
n=\frac{\hbar^{2} k_{F}^{2}}{m} \frac{\mathcal{V}^{2}}{(2 \pi)^{6}} N_{S I} I_{\nu},
$$

onde $\mathcal{V}$ é o volume da caixa, $N_{I S}$ é o número de autoestados de spin e isospin e $I_{\nu}=\int I d \nu$. O número de autoestados de spin e isospin é calculado através dos coeficientes de Clebsch-Gordan ou as funções de Racah nas expressões obtidas para $Q \mathcal{H} P$, tanto para o canal $\{000\}$ como $\{100\}$. Para obter os histogramas do capítulo 2 é só integrar $I$ em intervalos de $\nu$ (neste caso o intervalo é de $0.5 \nu$ ). 


\section{Apêndice D}

\section{Tratamento de $M_{q R}$ e $g_{f q}$}

Definimos $M_{q R}$ e $g_{f q}$ como

$$
\begin{aligned}
M_{q R} & \equiv\left\langle\tilde{q}\left|V_{Q P}^{(-)} \mathcal{G}_{P P}^{o p t(-)} \mathcal{O}_{e e^{\prime}}\right| \Phi_{i}\right\rangle \\
g_{f q} & \equiv\left\langle\tilde{\chi}_{f}^{(-)}\left|V_{Q P}^{(-)}\right| q\right\rangle
\end{aligned}
$$

Na segunda expressão acima podemos introduzir um conjunto completo de canais abertos $c_{1}$

$$
\begin{aligned}
g_{f q} & =\left\langle\tilde{\chi}_{f}^{(-)}\left|\sum_{c_{1}} \int d \vec{r}_{1}\right| \vec{r}_{1} c_{1}\right\rangle\left\langle\vec{r}_{1} c_{1}\left|V_{Q P}^{(-)}\right| q\right\rangle \\
& =\sum_{c_{1}} \int d \vec{r}_{1}\left\langle\tilde{\chi}_{f}^{(-)} \mid \vec{r}_{1} c_{1}\right\rangle\left\langle\vec{r}_{1} c_{1}\left|V_{Q P}^{(-)}\right| q\right\rangle \\
& =\sum_{c_{1}} \int d \vec{r}_{1} \chi_{f c_{1}}^{*}\left(\vec{r}_{1}\right) V_{q c_{1}}\left(\vec{r}_{1}\right) .
\end{aligned}
$$

A equação (D.1) é também escrita como:

$$
M_{q R} \equiv\left\langle\tilde{q}\left|V_{Q P}^{(-)} \frac{1}{E-H_{P P}-H_{P Q} \frac{1}{E-H_{Q Q}+2 I} H_{Q P}} \mathcal{O}_{e e^{\prime}}\right| \Phi_{i}\right\rangle
$$


Agora podemos tratar $\mathcal{G}_{P P}^{\text {opt(-) }}$. Introduzindo um conjunto completo com as condições de contorno apropriadas, ou seja, uma função de onda óptica com canais abertos $c_{2}$ e energia $E^{\prime}, \chi_{c_{2}}\left(E^{\prime}\right)$, obtemos

$$
M_{q R} \equiv\left\langle\tilde{q}\left|V_{Q P}^{(-)} \frac{1}{2 \pi} \sum_{c_{2}} \int d E^{\prime} \frac{\left|\chi_{c_{2}}^{(+)}\left(E^{\prime}\right)\right\rangle\left\langle\tilde{\chi}_{c_{2}}^{(+)}\left(E^{\prime}\right)\right|}{E-E^{\prime}-\imath \epsilon} \mathcal{O}_{e e^{\prime}}\right| \Phi_{i}\right\rangle .
$$

Novamente podemos colocar na expressão acima conjuntos completos equivalentes ao usado na equação (D.3),

$$
\begin{aligned}
M_{q R}= & \left\langle\tilde{q}\left|V_{Q P}^{(-)} \sum_{c_{1}} \int d \vec{r}_{1}\right| \vec{r}_{1} c_{1}\right\rangle\left\langle\vec{r}_{1} c_{1}\right| \\
& \times \frac{1}{2 \pi} \sum_{c_{2}} \int d E^{\prime} \frac{\left|\chi_{c_{2}}^{(+)}\left(E^{\prime}\right)\right\rangle\left\langle\tilde{\chi}_{c_{2}}^{(+)}\left(E^{\prime}\right)\right|}{E-E^{\prime}-\imath \epsilon} \sum_{c_{0}} \int d \vec{r}_{0}\left|\vec{r}_{0} c_{0}\right\rangle\left\langle\vec{r}_{0} c_{0}\left|\mathcal{O}_{e e^{\prime}}\right| \Phi_{i}\right\rangle \\
= & \sum_{c_{1}} \int d \vec{r}_{1}\left\langle\tilde{q}\left|V_{Q P}^{(-)}\right| \vec{r}_{1} c_{1}\right\rangle \\
& \times \sum_{c_{0}} \int d \vec{r}_{0} \frac{1}{2 \pi} \sum_{c_{2}} \int d E^{\prime} \frac{\left\langle\vec{r}_{1} c_{1} \mid \chi_{c_{2}}^{(+)}\left(E^{\prime}\right)\right\rangle\left\langle\tilde{\chi}_{c_{2}}^{(+)}\left(E^{\prime}\right) \mid \vec{r}_{0} c_{0}\right\rangle}{E-E^{\prime}-\imath \epsilon}\left\langle\vec{r}_{0} c_{0}\left|\mathcal{O}_{e e^{\prime}}\right| \Phi_{i}\right\rangle \\
= & \sum_{c_{1}} \int d \vec{r}_{1} V_{q c_{1}}\left(\vec{r}_{1}\right) \frac{1}{2 \pi} \sum_{c_{0}} d \vec{r}_{0} \mathcal{G}_{c_{0} c_{1}}^{(E)}\left(\vec{r}_{0}, \vec{r}_{1}\right) \mathcal{M}_{R c_{0}}\left(\vec{r}_{0}\right) \\
= & \sum_{c_{1}} \int d \vec{r}_{1} V_{q c_{1}}\left(\vec{r}_{1}\right) m_{R c_{1}}^{*}\left(\vec{r}_{1}\right)
\end{aligned}
$$

onde

$$
\begin{aligned}
V_{q c_{1}}\left(\vec{r}_{1}\right) & \equiv\left\langle\tilde{q}\left|V_{Q P}^{(-)}\right| \vec{r}_{1} c_{1}\right\rangle \\
\mathcal{M}_{R c_{0}}\left(\vec{r}_{0}\right) & \equiv\left\langle\vec{r}_{0} c_{0}\left|\mathcal{O}_{e e^{\prime}}\right| \Phi_{i}\right\rangle \\
\mathcal{G}_{c_{0} c_{1}}^{(E)}\left(\overrightarrow{r_{0}}, \overrightarrow{r_{1}}\right) & \equiv \sum_{c_{2}} \int d E^{\prime} \frac{\left\langle\vec{r}_{1} c_{1} \mid \chi_{c_{2}}^{(+)}\left(E^{\prime}\right)\right\rangle\left\langle\tilde{\chi}_{c_{2}}^{(+)}\left(E^{\prime}\right) \mid \vec{r}_{0} c_{0}\right\rangle}{E-E^{\prime}-\imath \epsilon} \\
m_{R c_{1}}^{*}\left(\vec{r}_{1}\right) & =\frac{1}{2 \pi} \sum_{c_{0}} \int d \overrightarrow{r_{0}} \mathcal{G}_{c_{0} c_{1}}^{(E)}\left(\vec{r}_{0}, \vec{r}_{1}\right) \mathcal{M}_{R c_{0}}\left(\vec{r}_{0}\right) .
\end{aligned}
$$

A parte lisa é dada por 


$$
\begin{aligned}
T_{f i} & =\left\langle\tilde{\chi}_{f}^{(-)}\left|\mathcal{O}_{e e^{\prime}}\right| \Phi_{i}\right\rangle \\
& =\left\langle\tilde{\chi}_{f}^{(-)}\left|\sum_{c_{0}} \int d \overrightarrow{r_{0}}\right| \overrightarrow{r_{0}} c_{0}\right\rangle\left\langle\overrightarrow{r_{0}} c_{0}\left|\mathcal{O}_{e e^{\prime}}\right| \Phi_{i}\right\rangle \\
& =\sum_{c_{0}} \int d \overrightarrow{r_{0}}\left\langle\tilde{\chi}_{f}^{(-)} \mid \overrightarrow{r_{0}} c_{0}\right\rangle\left\langle\overrightarrow{r_{0}} c_{0}\left|\mathcal{O}_{e e^{\prime}}\right| \Phi_{i}\right\rangle \\
& =\sum_{c_{0}} \int d \overrightarrow{r_{0}} \chi_{f c_{0}}^{*}\left(\overrightarrow{r_{0}}\right) \mathcal{M}_{R c_{0}}\left(\overrightarrow{r_{0}}\right) .
\end{aligned}
$$




\section{Referências}

[1] P. Barreau et al., Nucl. Phys. A402(1983)515.

[2] R. Altemus et al., Phys. Rev. Lett. 44(1980)965.

[3] M. Deady et al., Phys. Rev. C28(1983)631.

[4] Z. E. Meziani et al., Phys. Rev. Lett. 52(1984)2130; 54(1985)1233.

[5] A. Hotta et al., Phys. Rev. C30(1984)87.

[6] C. Marchand et al., Phys. Lett. B153(1985)29.

[7] T. deForest and J. D. Walecka, Adv. Phys. 57(1966)1; T. deForest, Ann. Phys. 45(1967))365.

[8] R. R. Whitney et al., Phys. Rev. C9(1974)2230; W. Czyż, Phys. Rev. 131(1963)2141.

[9] G. Orlandini and M. Traini, Rep. Prog. Phys. 54(1991)257.

[10] J. W. Negele, Rev. Mod. Phys. 54(1982)913.

[11] "Unifield Theory of Nuclear Models", G. Brown, North-Holland - Amsterdam, 1964.

[12] K. Takayanagi, K. Shimizu and A. Arima, Nucl. Phys. A477(1988)20; A481(1988)313. 
[13] M. Buballa, S. Drożdż, S. Krewald and J. Speth, Ann. Phys. 208(1991)346.

[14] G. Co', K. F. Quader, R.D. Smith and J. Wambach, Nucl. Phys. A485(1988)61;

S. Drożdż, M. Buballa, S. Krewald and J. speth, Nucl. Phys. A501(1989)487.

[15] S. Drożdż, S. Nisshizaki, J. speth and J. Wambach, Phys. Rep. 197(1990)1.

[16] G. DoDang and N. Van Gial, Phys. Rev. C31(1984)731.

[17] J. V. Noble, Phys. Rev. Lett. 46(1981)412.

[18] J. W. Van Orden and T. W. Donnelly, Ann. Phys. 131(1981)451.

[19] W. Hauser and H. Feshbach, Phys. Rev. 87(1952)366.

[20] P. A. Moldauer, Phys. Rev. 123(1961)968; 129(1963)754; B135(1964)642; G. R. Satchler, Phys. Rev. Lett. 7(1963)55.

[21] M. Kawai, A. K. Kerman and K. W. McVoy, Ann. Phys. 75(1973)156.

[22] G. van der Steenhoven et al., Nucl. Phys. A484(1988)445; A480(1988)547.

[23] A. K. Kerman and K. W. McVoy, Ann. Phys. 122(1979)197.

[24] “Quantum Theory of Many-Particle Systems”, A. L. Fetter and J. K. Walecka, McGraw-Hill (1971).

[25] "Bases Microscópicas da Estrutura Nuclear" (notas de aula), A. F. R. de Toledo Piza, (1983); A. F. R. de Toledo Piza and A. K. Kerman, Ann. Phys. 43(1967)363.

[26] V. Klemt, S. A. Moszlowski and J. Speth, Phys. Rev. C14(1976)302.

[27] K. Nakayama, S. Krewald, J. Speth and W. G. Love, Nucl. Phys. A431(1984)419. 
[28] S. Kopp, A. Szczurek, G. Co' and S. Krewald, Nucl. Phys. A570(1994)599.

[29] K. Nakayama, S. drozdz, S. Krewald and J. Speth, Nucl. Phys. A470(1987)573.

[30] G. P. Co' and S. Krewald, Nucl. Phys. A433(1985)392; G. A. Rinker and J. Speth, Nucl. Phys. A306(1978)360.

[31] "The Nuclear Many-Body Problem", P. Ring and P. Schuck, Springer-Verlag New York Inc, 1980; R. Bauer, K. Ebert, P. Ring, W. Theis, E. Werner and W. Wild, Z. Physik A274(1975)41; P. Ring and J. Speth, Nucl. Phys. A235(1974)315.

[32] A. Mariano, E. Bauer, F. Krmpotić and A. F. R. de Toledo Piza, Phys. Lett. B268(1991)332.

[33] “Angular Momentum", D. M. Brink and G. R. Satchler, Clarendon Press Oxford, 1962. 